Portland State University

PDXScholar

Spring 4-30-2014

\title{
Anisotropy of the Reynolds Stress Tensor in the Wakes of Counter-Rotating Wind Turbine Arrays
}

Nicholas Michael Hamilton

Portland State University

Follow this and additional works at: https://pdxscholar.library.pdx.edu/open_access_etds

Part of the Energy Systems Commons, and the Power and Energy Commons Let us know how access to this document benefits you.

\section{Recommended Citation}

Hamilton, Nicholas Michael, "Anisotropy of the Reynolds Stress Tensor in the Wakes of Counter-Rotating Wind Turbine Arrays" (2014). Dissertations and Theses. Paper 1848.

https://doi.org/10.15760/etd.1847

This Thesis is brought to you for free and open access. It has been accepted for inclusion in Dissertations and Theses by an authorized administrator of PDXScholar. Please contact us if we can make this document more accessible: pdxscholar@pdx.edu. 
Anisotropy of the Reynolds Stress Tensor in the Wakes of Counter-Rotating Wind Turbine Arrays

by

Nicholas Michael Hamilton

A thesis submitted in partial fulfillment of the requirements for the degree of

\author{
Master of Science \\ in \\ Mechanical Engineering
}

Thesis Committee:

Raúl Bayoán Cal, Chair

Gerald Recktenwald

Mark Weislogel

Portland State University

2014 


\begin{abstract}
A wind turbine array was constructed in the wind tunnel at Portland State university in a standard Cartesian arrangement. Configurations of the turbine array were tested with rotor blades set to rotate in either a clockwise or counter-clockwise sense. Measurements of velocity were made with stereo particle-image velocimetry. Mean statistics of velocities and Reynolds stresses clearly show the effect of direction of rotation of rotor blades for both entrance and exit row turbines. Rotational sense of the turbine blades is visible in the mean spanwise velocity $W$ and the Reynolds shear stress $-\overline{v w}$. The normalized anisotropy tensor was decomposed yielding invariants $\eta$ and $\xi$, which are plotted onto the Lumley triangle. Invariants of the normalized Reynolds stress anisotropy tensor indicate that distinct characters of turbulence exist in regions of the wake following the nacelle and the rotor blade tips. Eigendecomposition of the tensor yields principle components and corresponding coordinate system transformations. Characteristic spheroids are composed with the eigenvalues from the decomposition yielding shapes predicted by the Lumley triangle. Rotation of the coordinate system defined by the eigenvectors demonstrates streamwise trends, especially trailing the top rotor tip and below the hub of the rotors. Direction of rotation of rotor blades is evidenced in the orientation of characteristic spheroids according to principle axes. The characteristic spheroids of the anisotropy tensor and their relate alignments varies between cases clearly seen in the inflows to exit row turbines. There the normalized Reynolds stress anisotropy tensor shows cumulative effects of the rotational sense of upstream turbines. Comparison between the invariants of the Reynolds
\end{abstract}


stress anisotropy tensor and terms from the mean mechanical energy equation indicate a correlation between the degree of anisotropy and the regions of the wind turbine wakes where turbulence kinetic energy is produced. The flux of kinetic energy into the momentum-deficit area of the wake from above the canopy is associated with prolate characteristic spheroids. Flux upward into the wake from below the rotor area is associate with oblate characteristic spheroids. Turbulence in the region of the flow directly following the nacelle of the wind turbines demonstrates more isotropy compared to the regions following the rotor blades. The power and power coefficients for wind turbines indicate that flow structures on the order of magnitude of the spanwise turbine spacing that increase turbine efficiency depending on particular array configuration. 


\section{Acknowledgements}

I would like to express my gratitude for the support of my thesis advisor, Raúl Bayoán Cal. The guidance and motivation I have received have been instrumental in conducting research and pushing toward greater things. The faculty of the Mechanical and Materials Engineering Department, especially those on the thesis committee, has continued to encourage exploration and offer support throughout my tenure at PSU. My colleagues in the turbulence research group deserve special thanks, as well. Elizabeth Camp, Matt Melius, and Dominic Delucia have all provided much needed assistance in the lab and helped in discussing ideas and confirming findings. Mat Krieske tolerated rants and ramblings next to a dry-erase board for many evenings, providing a space for me to talk my way through difficult parts of the analysis. Finally, my family and loved ones have always been available to keep me fed and healthy, and to provide enough distraction for inspiration to sneak in around the edges. 


\section{Contents}

$\begin{array}{lll}\text { Abstract } & \text { i }\end{array}$

Acknowledgements iii

List of Tables $\quad$ vi

List of Figures vii

1 Introduction 1

2 Theory $\quad 8$

2.1 Equations of motion $\ldots \ldots \ldots \ldots$

2.2 Reynolds stress anisotropy . . . . . . . . . . . . . . . . . 10

2.3 Power production of wind turbines . . . . . . . . . . . . . . 15

3 Experimental Setup $\quad 18$

3.1 Overview of experiment $\ldots \ldots \ldots \ldots \ldots$

3.2 Flow measurement system $\ldots \ldots \ldots \ldots \ldots$. . . . . . . . . 21

3.3 Wind turbine models . . . . . . . . . . . . . . . . . . 24

3.4 Torque sensing system $\ldots \ldots \ldots \ldots \ldots \ldots \ldots$ 
4 Results $\quad 28$

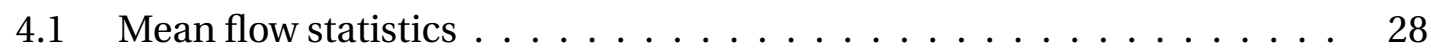

4.2 Analysis of anisotropy $\ldots \ldots \ldots \ldots \ldots$

4.3 Rotation of principle coordinate system . . . . . . . . . . . . . 51

4.4 Flux and production of kinetic energy . . . . . . . . . . . . . . 63

4.5 Power and efficiency $\ldots \ldots \ldots \ldots \ldots \ldots$

5 Conclusions $\quad 73$

$\begin{array}{lr}\text { Bibliography } & 79\end{array}$ 


\section{List of Tables}

2.1 Special states of turbulence given on the Lumley triangle. Limits of the Reynolds stress anisotropy tensor are discussed in terms of matrix invariants and eigenvlaues. Spheroidal radii correspond to principle stresses from the eigenvalue decomposition given in Equation (2.10). . . . . . . . 13

4.1 Park efficiency according to array configuration [10] . . . . . . . . . 71 


\section{List of Figures}

1.1 Global production from wind energy. Figure reproduced from [41]. . . 2

$2.1 \quad$ Lumley triangle showing limits of invariants $\eta$ and $\xi \ldots \ldots \ldots \ldots$

2.2 Characteristic shapes of $b_{i j}$. Shapes for special cases correspond to those in Table $2.1 . \ldots \ldots \ldots \ldots \ldots \ldots$

3.1 Experimental setup shown in wind tunnel test section. Dimensions are for reference only; image not to scale. . . . . . . . . . . . . . .

3.2 Characteristic quantities pertaining to the approach flow boundary layer of the experiment. All measurements taken upstream of wind turbine array. The streamwise coordinate has been normalized with the turbine rotor diameter. $\ldots \ldots \ldots \ldots \ldots \ldots$

3.3 Configurations of the wind turbine array tested in the PSU wind tunnel. The colored rotation vector indicates rotational sense of the turbine rotors according to the right-hand-rule. Red vectors indicate counter clockwise rotation; blue vectors indicate clockwise rotation. Dashed lines indicate SPIV measurement planes. . . . . . . . . . . . . . . 22

3.4 Detail view of SPIV measurement areas located directly upstream and downstream of wind turbine models. . . . . . . . . . . . . . . . . 23 
3.5 Technical drawing of model wind turbines used in experiments. All dimensions are in millimeters. The mounting plate shown in 3.5(A) spans the full width of the wind tunnel; only a small section is shown.

3.6 Torque sensor used in the experiment. Reflective tape show on the topmost rotor blade in 3.6(B) was used in the measurements of angular velocity by the remote optical sensor. . . . . . . . . . . . . .

4.1 Positions of contour plots of mean velocities, $U$ and $W$ in Figures 4.2

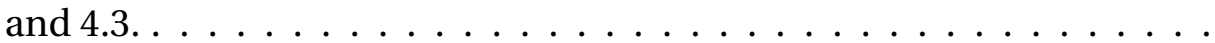

4.2 Mean streamwise velocity normalized by the inflow velocity at hub height, $U / U_{\text {hub }} \ldots \ldots \ldots \ldots \ldots \ldots \ldots \ldots \ldots$

4.3 Mean spanwise velocity normalized by the inflow velocity at hub height, $W / U_{\text {hub. }} \ldots \ldots \ldots \ldots \ldots \ldots \ldots \ldots \ldots \ldots \ldots \ldots \ldots \ldots$

$4.4 \quad$ Normalized Reynolds shear stress $-\overline{u v} / U_{\text {hub }}^{2} \ldots \ldots \ldots \ldots$. . . . . 34

$4.5 \quad$ Normalized Reynolds shear stress $-\overline{v w} / U_{\text {hub }}^{2} \ldots \ldots \ldots \ldots$

4.6 In-plane flux of turbulence kinetic energy, $F_{12}=-\overline{u v} U . \ldots . \ldots 36$

$4.7 \quad$ In-plane production of turbulence kinetic energy, $P_{12}=-\overline{u \nu} \frac{\partial U}{\partial y} \ldots \ldots \quad 37$

4.8 Plots of second invariant of the normalized anisotropy tensor, $\eta$ corresponding to the ordinate of the Lumley triangle. The degree of anisotropy is associated with $\eta \ldots \ldots \ldots$. . . . . . . . . 40

4.9 Plots of third invariant of the normalized anisotropy tensor, $\xi$ corresponding to the abscissa of the Lumley triangle. Characteristic shapes of Reynolds stress anisotropy are described by $\xi$. . . . . . . . . . . 
4.10 Lumley triangles for each of the measurement cases. Points in black and red represent measurement windows directly upstream and downstream, respectively. Every fifth point is plotted for clarity. . . . . . . . .

4.11 AIMs for average invariants in areas of interest of exit row turbine wakes. Averaging areas are shown in Subfigure 4.11(A). Average invariants for all cases (o - uniform, $\square$ - row-by-row, $\diamond-$ column-bycolumn, * - checkerboard) are shown in upstream (black) and downstream measurement (red) windows. . . . . . . . . . . . . .

4.12 Characteristic spheroids in selected locations of the wake of the exit row turbine of the uniform case $\ldots \ldots \ldots \ldots \ldots$

4.13 Extreme degrees of anisotropy in the wake of exit row wind turbine of the row-by-row case defined by extreme values of $\eta$. At left is the least isotropic spheroid, in the center is the most isotropic spheroid. The AIM in shows the invariants for each point as a star $(*)$ for the most isotropic state and a square $(\square)$ for the least isotropic state. . . . . . . . 50

4.14 Unfiltered rotational angles of principle axes about Cartesian axes. Data pertains to the exit row of the uniform case. From left to right are rotations about the $x, y$, and $z$ axes, $\theta_{x}, \theta_{y}$, and $\theta_{z}$ respectively. . .

4.15 Octants of the Cartesian coordinate system defined by the sign of standard vectorial components and rotated coordinate system and characteristic vectors of both the Cartesian system and the principle axes. . . 
4.16 Percent occupation of principle axes in Cartesian octants. Subfigures 4.16(B) through 4.16(D) show the occupation of principle axes in areas of interest in the wake and Subfigure 4.16(A) shows the occupation for the entire measurement area. . . . . . . . . . . . . . 55

4.17 Residence of characteristic vector $\vec{r}$ in Cartesian octants after application of treatment to Euler angles. . . . . . . . . . . . . . 5

4.18 Euler angles (about default axes) of rotation of principle coordinate system. Columns from left are $\theta_{x}, \theta_{y}$, and $\theta_{z}$ (rotations about the streamwise, wall-normal, and spanwise coordinates, respectively). . .

4.19 Rotated characteristic spheroids for exit row turbine wakes. Fields are tilted along the $x$ axis to better illustrate spheroids. Only one hundredth of the total number of spheroids are plotted for clarity. . . . .

4.20 Scatter plots of the flux and production of kinetic energy against the invariants of $b_{i j} \ldots \ldots \ldots \ldots \ldots \ldots \ldots \ldots \ldots \ldots \ldots \ldots \ldots \ldots \ldots$

4.21 From left are the algebraic signs of $F_{12}$ and $\xi$ and their regions of overlap. In the right column, overlaps are positive or negative and matching (in white and black, respectively) or not matching (in gray). . . . . . .

4.22 Threshold plots of $P_{12}$ and $\eta$ in the left and center columns respectively. The right column shows overlapping areas as positive or negative and matching (in white and black, respectively) or not matching (in gray).

4.23 Power curves and curves of power coefficient for all exit row turbine

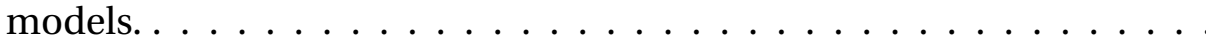




\section{Chapter 1}

\section{Introduction}

The United States Department of Energy has adopted a goal to generate $20 \%$ of the nation's electricity from wind power by 2030 [22]. Achieving the "20\% Wind Scenario" in 2030 requires acceleration of the current rate of wind project developments including both on-shore and off-shore wind parks [37]. Meeting this goal today would dictate that wind energy account for approximately $600 \mathrm{GW}$ of electricity generation $(20 \%$ of the 3.8 TW generated in the U.S. in 2013). The current installed capacity of wind farms in the U.S. is on the order of $61 \mathrm{GW}$, only $1.6 \%$ of current generation.

Many countries have planned or implemented similar goals to provide energy through renewable sources in accord with the Kyoto protocols outlined in 1997 [25]. The plan by the U.S. to reach the $20 \%$ goal mirrors that of the European Union, as described in a 2013 report [12]. Looking even farther forward, there are many municipalities in the U.S. and abroad that have goals to produce all of their energy by local and sustainable means. One such example states that, "Local governments across Japan are seeking to supply their regions with $100 \%$ renewable energy," [40]. These serve to illustrate the importance of continued research into wind energy. While these goals are ambitious, the global wind energy sector has demonstrated continued and accelerating growth. The installed wind power capacity globally has increased more than 250 
GW in the ten years from 2002 to 2012, as shown in Figure 1.1.

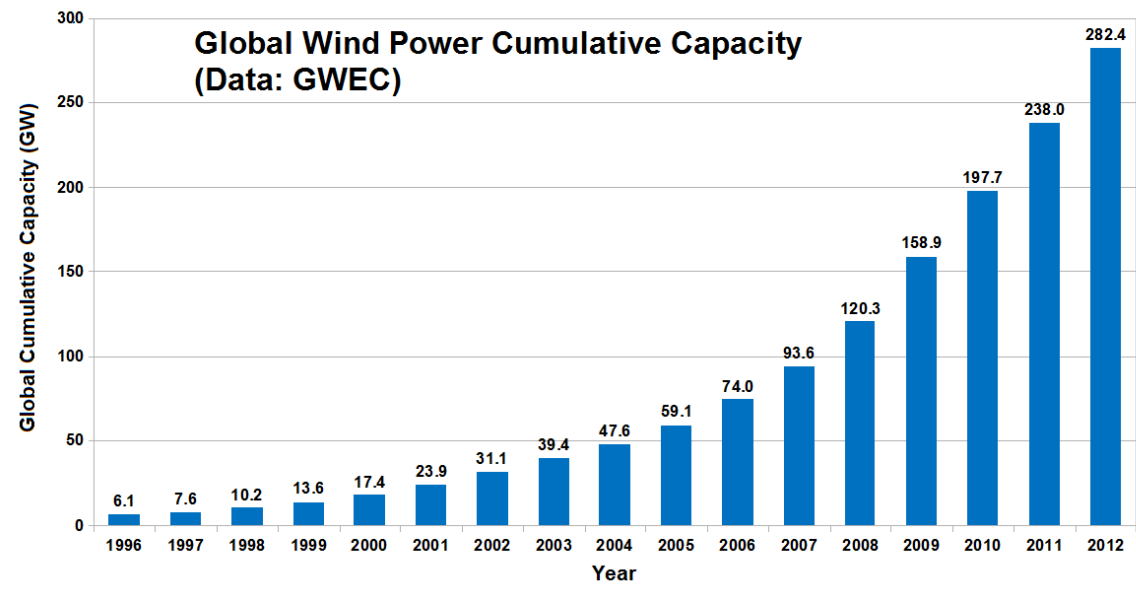

Figure 1.1: Global production from wind energy. Figure reproduced from [41].

Wind energy is not in itself a new field of research. Wind turbines have been studied to a large extent on an individual basis $[1,38,39]$. A study by Chamorro and PortéAgel [6] found that characteristics in the wake of an individual wind turbine depend on the surface roughness in the approach flow. Mean velocity was assessed using hot-wire anemometry in order to characterize the cross-sectional distribution of mean velocity, turbulence intensity, and kinematic shear stress. The spatial distribution of the velocity deficit and the turbulence intensity, important factors affecting turbine power generation and fatigue loads in wind turbine rotor blades and hubs, were found to exhibit asymmetry in the wakes. The distribution and intensity of turbulence in the wake was found to be stronger over the rougher surfaces, where the incoming flow is less uniform at turbine height.

Research interest in wind turbine arrays has increased as characterizing the turbulent flow in the wind turbine canopy has been shown to be a complex challenge and wind power production becomes more attractive. The energy produced by a wind tur- 
bine can be correlated to the difference in the flux of turbulence kinetic energy at top and bottom rotor tips [4]. In large arrays especially, the global kinetic energy entrainment is downward from the free flow above the wind turbines into the main canopy layer. The formulation of the flux of kinetic energy suggests that energy containing structures, especially those associated with the top tip of the rotor area, are responsible for the entrainment of energy downward into the main turbine canopy area [17]. Investigation of the component quantities of the flux of kinetic energy is of continued interest in studies pertaining to wind turbine arrays.

With the decrease of cost and the increase of computational power, numerical simulations of wind arrays have become more complex $[2,9,13,27]$. Many models use self-similar velocity deficit profiles obtained from experimental and theoretical work. The growth rate of wakes in turbine arrays is generally attributed to the level of turbulence encountered, which is amplified by the mean shear in the wind turbine canopy layer. Often, the momentum deficit predicted in wind turbine wakes is obtained from global conservation principles.

In many simulations, large wind farms are considered as a series of solid bodies or disks that increase the effective surface roughness for the atmospheric boundary layer $(\mathrm{ABL})$ and decrease mean wind speeds near the ground. The spacing of devices within an array has been optimized for efficiency [24]. Increasing the turbine-land area density (i.e. decreasing the average wind-turbine spacing) often has an opposite effect on the total extracted power per turbine. The optimization undertaken by Meyers and Meneveau [24] addresses the problem of wind-turbine spacing in wind farms, where the optimal spacing is a product of economical constraints and the performance of turbines within the array. The study showed that the optimal spacing for turbines 
based on performance may be as large as 15 rotor diameters in the streamwise direction rather than the 6 or 7 diameters typically found in real installations.

It is now common for wind farms to be designed with numerical simulations as GIS wind data is already readily available. In the ABL and the wind turbine canopy layer, especially in the near wake, there exists some anisotropy in the fluid stresses due to the swirl imparted to the flow from the passage of the rotor blades [34]. It is for this reason that many of the more sophisticated wind farm simulations opt for a more computationally expensive model. In the academic setting, wind farms are being investigated through large eddy simulations $[5,24]$ in which terrains are simplified and various arrangements of the arrays can be tested fairly quickly. Models balancing the transport equations of the Reynolds stresses are far more effective at representing the anisotropy of turbulence than the more computationally efficient RANS codes that balance the transport of turbulence kinetic energy and dissipation, $(k-\epsilon$ models), or those that balance the turbulence kinetic energy and vorticity, ( $k-\omega$ models) [28].

Return-to-isotropy models, introduced by Rotta [29], have been employed to represent the asymptotic behavior of the stresses even in the inner boundary layer. The Rotta model is not commonly used in wind turbine or array simulations, however, explicit algebraic models are fairly common in second order closure schemes of either $k-\epsilon$ or $k-\omega$ models [14]. The anisotropic properties of the Reynolds stresses with regards to wind turbines has yet to be fully explored in experiments and simulations.

The terminology related to the normalized Reynolds stress anisotropy tensor was reviewed and clarified by Simonsen and Krogstad [31]. Here the principle components resulting from an eigenvalue decomposition were related to spheroidal structures characterizing the anisotropy of turbulence. Additionally it was detailed that the 
transformations for structures of vorticity have an inverse relationship to those for turbulent stresses. The eigenvectors resulting from the decomposition of the normalized anisotropy tensor form an orthonormal basis describing a coordinate system corresponding to the eigenvalues that has been rotated from the original Cartesian system.

Tidal stream turbines have been analyzed to some extent by Tedds, et al. [35]. In that work, Acoustic Doppler Velocimetry measurements were made in the wake of a model tidal stream turbine. The normalized Reynolds stress anisotropy tensor was then decomposed according to the procedure outlined by Choi and Lumley [8] and the resulting invariants plotted in the Lumley triangle. The work demonstrated a tendency toward oblate spheroids characterizing the anisotropy of turbulence in far wake. Although designed to function in water rather than the $\mathrm{ABL}$, a tidal stream turbine is geometrically similar to a horizontal axis wind turbine like those commonly deployed in large arrays.

Investigating a flat-plate boundary layer, it was found by Mestayer [23] that there is consistent evidence that local isotropy exists at scales smaller than twenty times the Kolmogorov microscale. This observation validates the assumption that local isotropy is expected in the dissipative range of scales but larger scales demonstrate anisotropy of turbulent stresses. Extending the work in boundary layers, Smalley et al. [32] showed that surface characteristics of the wall have significant effects on the balance of stresses. Their data point to the normal stresses being more isotropic over the rough surfaces than on a smooth wall. Later work by Leonardi [21] confirmed through direct numerical simulations of a channel flow that the Reynolds stress anisotropy tensor and its invariants show a closer approach to isotropy over the rough wall than over a smooth wall. 
Little experimentation has focussed on the anisotropy of turbulence in wind turbine arrays. Work by Smyth and Moum [33] determined the form of the Reynolds stress tensor for atmospheric flows. The results of that work forms a basis for comparison and validation of later simulation results. Notable computational work regarding the anisotropy of turbulence in the wakes of wind turbines is presented by Gómez et al. [14]. The model uses a second-order closure model with explicit algebraic models for the components of the turbulent stress tensor. The model is able to reproduce behavior seen in atmospheric turbulence. Further simulations were conducted by Jimenez et al. [18]. There a similar model was used to that of Gómez with the addition of analytical expressions for the estimation of an upper limit of the global turbulence kinetic energy, $k$.

A number of studies have been conducted regarding counter-rotation of rotors of horizontal axis wind turbines. One study [36] consisted of a wind tunnel experiment wherein a counter-rotating device was constructed and tested, demonstrating the technical possibility of counter-rotating type wind powered generators. A simulation was conducted regarding the relative size and optimum placement of a two-stage wind turbine [19] where the rotors were investigated through momentum theory for an experimental wake model. Similarly, a simulation of the performance of a wind turbine with a pair of counter rotating rotors was undertaken by Shen et al. [30]. The simulation made use of the commercial simulation package EllipSys3D, finding that Production can be increased to about $43.5 \%$ as compared to a wind turbine with a single rotor. The simulation consisted of a single device excluding the interaction of wakes or the formation of a developed turbine canopy.

The current work compares the turbulence in measurement planes directly up- 
stream and downstream of wind turbines in various configurations of an array in which the rotational sense of the rotors is varied. The Reynolds stress anisotropy tensor is decomposed into its principle components and related coordinates. Invariants of the anisotropy tensor are plotted on the Lumley triangle in order to assess the state of turbulence in the wakes. The invariants of the normalized Reynolds stress anisotropy tensor are compared to the flux of kinetic energy and the production of turbulence, as these are quantities of importance in the wind turbine canopy layer. The resupply of energy to the canopy layer of a wind turbine array is not yet fully understood. This research adds to the body of work characterizing the turbulence in the wakes of wind turbines, providing insight to the anisotropy of turbulence as a function of rotational direction of rotor blades. The results presented may also serve as a means of validation of numerical simulation of wind farms. 


\section{Chapter 2}

\section{Theory}

\subsection{Equations of motion}

The Reynolds averaged Navier-Stokes equations for a wind turbine canopy or wake are

$$
U_{j} \frac{\partial U_{i}}{\partial x_{j}}=-\frac{1}{\rho} \frac{\partial P}{\partial x_{i}}-\frac{\partial \overline{u_{i} u_{j}}}{\partial x_{j}}-\bar{f}_{x}
$$

In the equation the time derivative term has been omitted through ensemble averaging of the equations. The time dependence of the fluid motion and momentum are assumed to be null values when averaged over time. It is further assumed that all measurement locations described in the system are sufficiently far from solid bodies to neglect viscous terms in the local momentum balance. In the equation, the term $\bar{f}_{x}$ represents the thrust force of the turbines in the flow. This term acts primarily in the streamwise direction, leading to the subscript $x$ rather than the standard index. In all following equations, the coordinates are designated as $x$ (streamwise), $y$ (wallnormal), and $z$ (spanwise) directions, respectively. Capital letters indicate mean quantities and lower case letters refer to zero-centered fluctuations about the mean. An overline is employed to indicate that an ensemble average of the product of two quantities has been taken. 
Multiplying by the mean velocity yields the mean mechanical energy equation

$$
U_{j} \frac{\partial \frac{1}{2} U_{i}^{2}}{\partial x_{j}}=-\frac{1}{\rho} U_{i} \frac{\partial P}{\partial x_{i}}+\overline{u_{i} u_{j}} \frac{\partial U_{i}}{\partial x_{j}}-\frac{\partial \overline{u_{i} u_{j}} U_{i}}{\partial x_{j}}-\mathscr{F}_{x_{i}} .
$$

The left hand side of the Equation (2.2) is comprised of the convective terms and is balanced on the right by the power added to the flow through pressure gradients, the production of turbulence kinetic energy, and the flux of turbulence kinetic energy, respectively. The last term of Equation (2.2) is representative of the power removed from the flow by the action of a wind turbine rotor.

Of critical consideration in the global energy balance is the Reynolds stress tensor, $\overline{u_{i} u_{j}}$. This tensor expresses the balance of fluid stresses at any given measurement location and in Equation (2.2) is a component of both the kinetic energy flux and the turbulence production. The symmetric stress tensor, $\overline{u_{i} u_{j}}$, is given by

$$
\overline{u_{i} u_{j}}=\left[\begin{array}{ccc}
\overline{u^{2}} & -\overline{u v} & -\overline{u w} \\
-\overline{u v} & \overline{v^{2}} & -\overline{v w} \\
-\overline{u w} & -\overline{v w} & \overline{w^{2}}
\end{array}\right] .
$$

The Reynolds stress tensor is symmetric, arising from the Reynolds averaging process. Terms on the diagonal are normal stresses and off-diagonal terms are shear stresses in the turbulent stress field. The turbulent kinetic energy $k$ is defined as half of the trace of $\overline{u_{i} u_{j}}$,

$$
k=\frac{1}{2}\left(\overline{u^{2}}+\overline{v^{2}}+\overline{w^{2}}\right) .
$$




\subsection{Reynolds stress anisotropy}

The balance of stresses within the turbulent flow field is of importance when considering terms from Equation (2.2). The Reynolds stresses determine which components are responsible for production of turbulent kinetic energy and in which direction flux of kinetic energy occurs. In order to quantify preferential directions of the turbulent stress field, it is useful to define the Reynolds stress anisotropy tensor $a_{i j}$ according to the original development by Rotta [29] as,

$$
a_{i j} \equiv \overline{u_{i} u_{j}}-\frac{2}{3} k \delta_{i j}
$$

where $k$ is the turbulent kinetic energy and $\delta_{i j}$ is the Kronecker delta. The anisotropy tensor can be normalized by the turbulent kinetic energy as

$$
\begin{aligned}
& b_{i j}=\frac{a_{i j}}{2 k}=\frac{\overline{u_{i} u_{j}}}{\overline{u_{k} u_{k}}}-\frac{1}{3} \delta_{i j}
\end{aligned}
$$

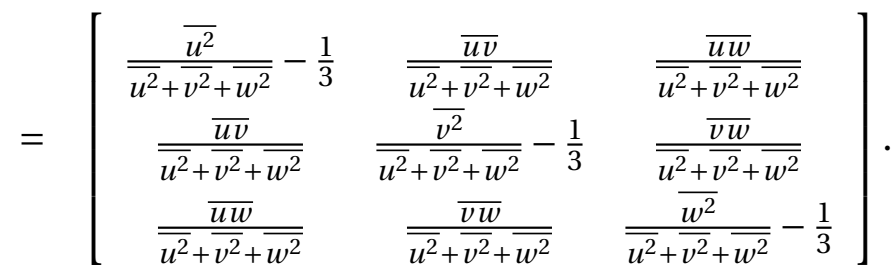

The normalized anisotropy tensor $b_{i j}$ has a zero trace as a consequence of its normalization. The traces of $b_{i j}^{2}$ and $b_{i j}^{3}$ are related to the invariants $\eta$ and $\xi$ of the anisotropy tensor by 


$$
\begin{aligned}
& 6 \eta^{2}=b_{i i}^{2}=b_{i j} b_{j i} \\
& 6 \xi^{3}=b_{i i}^{3}=b_{i j} b_{j k} b_{k i} .
\end{aligned}
$$

An eigenvalue decomposition of $b_{i j}$ yields three eigenvalues whose sum is necessarily zero, following the trace of the kernel $b_{i j}$, and a set of eigenvectors that correspond to an optimal basis describing the vector space of $b_{i j}$

$$
b_{i j}=\Sigma_{i j} \lambda_{i} \Sigma_{i j}^{-1}
$$

The decomposition outlined in Equation (2.8) transforms the symmetric second-order tensor of the normalized Reynolds stress anisotropy into a set of principle components $\lambda_{i}$ and associated principle axes $\Sigma_{i j}$. Given that the principle axes form an orthonormal basis, their composition formulates a coordinate system as

$$
\Sigma_{i j}=\left\langle x^{\prime}, y^{\prime}, z^{\prime}\right\rangle
$$

Here $x^{\prime}, y^{\prime}$, and $z^{\prime}$ are axes of the optimal coordinate system associated with $b_{i j}$. In its principle axes (denoted below with a caret), $b_{i j}$ reduces to principle components as

$$
\hat{b}_{i j}=\left[\begin{array}{ccc}
\lambda_{1} & 0 & 0 \\
0 & \lambda_{2} & 0 \\
0 & 0 & -\lambda_{1}-\lambda_{2}
\end{array}\right],
$$

where $\lambda_{i}$ are the eigenvalues of $b_{i j}$ from Equation (2.8). 
The invariants $\eta$ and $\xi$ can be expressed in terms of the eigenvalues directly as

$$
\begin{aligned}
\eta^{2} & =\frac{1}{3}\left(\lambda_{1}^{2}+\lambda_{1} \lambda_{2}+\lambda_{2}^{2}\right) \\
\xi^{3} & =-\frac{1}{2} \lambda_{1} \lambda_{2}\left(\lambda_{1}+\lambda_{2}\right)
\end{aligned}
$$

Invariants of the normalized Reynolds stress anisotropy tensor are related to the degree of anisotropy $(\eta)$ and the characteristic shape described by the eigenvalues $(\xi)$.

The invariants of $b_{i j}$ described by either Equation (2.7) or (2.11) can be shown graphically in the anisotropy invariant map (AIM), commonly known as the Lumley triangle [8]. In the following text, the terms AIM and Lumley triangle are used interchangeably. In Figure 2.1, theoretical limits are shown as vertices or edges of the triangle and represent various states or special cases of turbulence. The limits of 'realizable' turbulence described in the Lumley triangle are detailed in Table 2.1.

The upper limit in the Lumley triangle describes two-component turbulence and arises as a consequence of the eigendecomposition. For this limit, the sum of the first two eigenvalues is, $\lambda_{1}+\lambda_{2}=1 / 3$. Written in terms of the invariants, the limit is described as $\eta=\left(1 / 27+2 \xi^{3}\right)^{1 / 2}$.

Considering that the outputs of the eigendecomposition of $b_{i j}$ are a set of principle components and associated vector basis, the anisotropic state of turbulence can be described by a characteristic spheroid whose radii correspond to the eigenvalues $\lambda_{i}$ and is rotated with respect to the original Cartesian coordinate system to one described by the eigenvectors in Equation (2.9). The shape of the characteristic spheroids correspond to the limits described in the rightmost column of Table 2.1. 


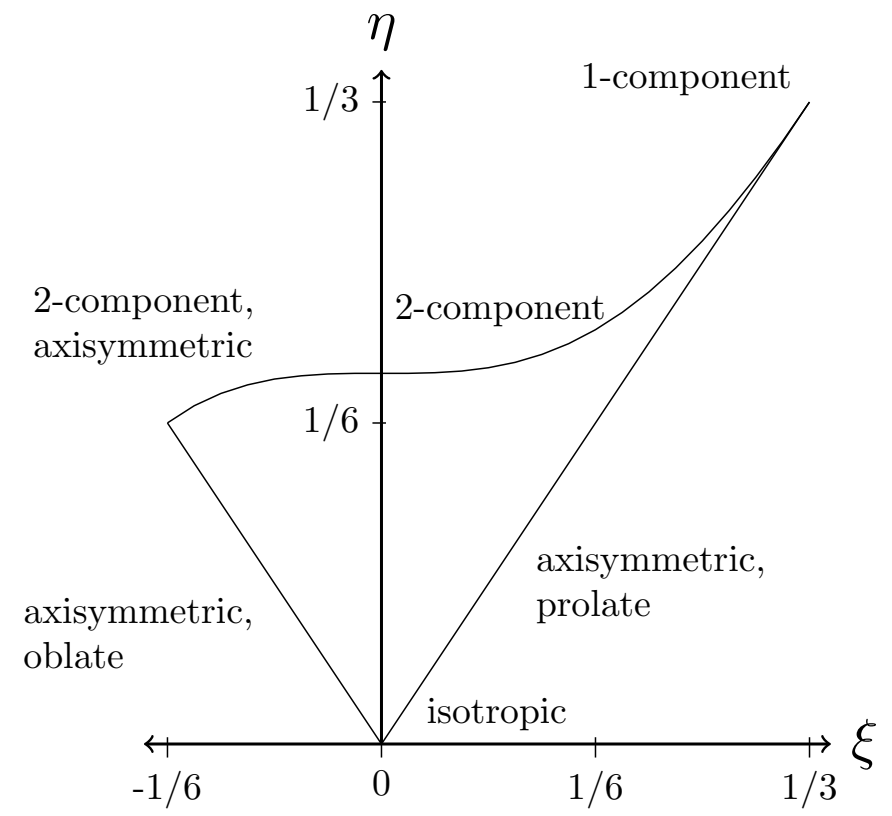

Figure 2.1: Lumley triangle showing limits of invariants $\eta$ and $\xi$.

Table 2.1: Special states of turbulence given on the Lumley triangle. Limits of the Reynolds stress anisotropy tensor are discussed in terms of matrix invariants and eigenvlaues. Spheroidal radii correspond to principle stresses from the eigenvalue decomposition given in Equation (2.10).

\begin{tabular}{|c|c|c|c|}
\hline $\begin{array}{c}\text { State of } \\
\text { turbulence }\end{array}$ & Invariants & $\begin{array}{l}\text { Eigenvalues } \\
\quad \text { of } b_{i j}\end{array}$ & $\begin{array}{l}\text { Shape of } \\
\text { spheroid }\end{array}$ \\
\hline Isotropic & $\xi=\eta=0$ & $\lambda_{i}=0$ & Sphere \\
\hline $\begin{array}{l}\text { Two-component } \\
\text { axisymmetric }\end{array}$ & $\xi=-\frac{1}{6}, \eta=\frac{1}{6}$ & $\lambda_{1}=\lambda_{2}=\frac{1}{6}$ & Disk \\
\hline One-component & $\xi=\eta=\frac{1}{3}$ & $\begin{array}{c}\lambda_{1}=\frac{2}{3} \\
\lambda_{2}=\lambda_{3}=-\frac{1}{3}\end{array}$ & Line \\
\hline $\begin{array}{c}\text { Axisymmetric } \\
\text { (one large eigenvalue) }\end{array}$ & $\xi=\eta$ & $-\frac{1}{3} \leq \lambda_{1}=\lambda_{2} \leq 0$ & $\begin{array}{l}\text { Prolate } \\
\text { spheroid }\end{array}$ \\
\hline $\begin{array}{c}\text { Axisymmetric } \\
\text { (one small eigenvalue) }\end{array}$ & $-\xi=\eta$ & $0 \leq \lambda_{1}=\lambda_{2} \leq \frac{1}{6}$ & $\begin{array}{l}\text { Oblate } \\
\text { spheroid }\end{array}$ \\
\hline Two-component & $\eta=\left(\frac{1}{27}+2 \xi^{3}\right)^{1 / 2}$ & $\lambda_{1}+\lambda_{2}=\frac{1}{3}$ & ellipse \\
\hline
\end{tabular}


The special cases of turbulence outlined in Table 2.1 are often used in scaling and theoretical development but are not often observed in experiments. It is often assumed that small scale turbulence (within the dissipative range) is isotropic, whereas the energetic scales rarely are. The two-component case described by the upper edge of the Lumley triangle is similarly rare in real flows. In contrast, axisymmetric turbulence is common in wakes or generated in periodic flows. Much of the observed turbulence presented here is axisymmetric to some degree and can be characterized by the shapes described in Table 2.1.

The characteristic shapes associated with axisymmetric turbulence are spheres, as in the case of isotropic turbulence, or either oblate or prolate spheroids, in the case of anisotropic turbulence. Oblate spheroids exhibit two eigenvalues that are equal (or very similar) and one eigenvalue that is small compared to the others. This results in a spheroid that is squeezed in one direction. Prolate spheroids show the opposite effect with one eigenvalue that is large compared to the other (equal or very similar) eigenvalues, resulting in a spheroid that is stretched in one direction.

The remaining limits of realizable turbulence include one- and two-dimensional turbulence. In the case of one-dimensional (or one-component) turbulence, denoted as the point on the upper-right corner of the Lumley triangle, the characteristic shape of turbulence can be thought of as a prolate spheroid stretched infinitely along its greatest eigenvalue, rendering the other radii zero in a normalized system. Onedimensional turbulence shows the least conformity between components and is the least invariant to rotation. Two dimensional turbulence can be thought of as the limit of oblate spheroids, wherein the one small eigenvalue is reduced to zero, and the characteristic shape becomes an ellipse. In two-dimensional axisymmetric turbulence, the 
characteristic shape is a circle and is invariant to rotation only on the axis defined by its null eigenvalue. Figure 2.2 shows characteristic spheroids corresponding to the special cases of turbulence described above.

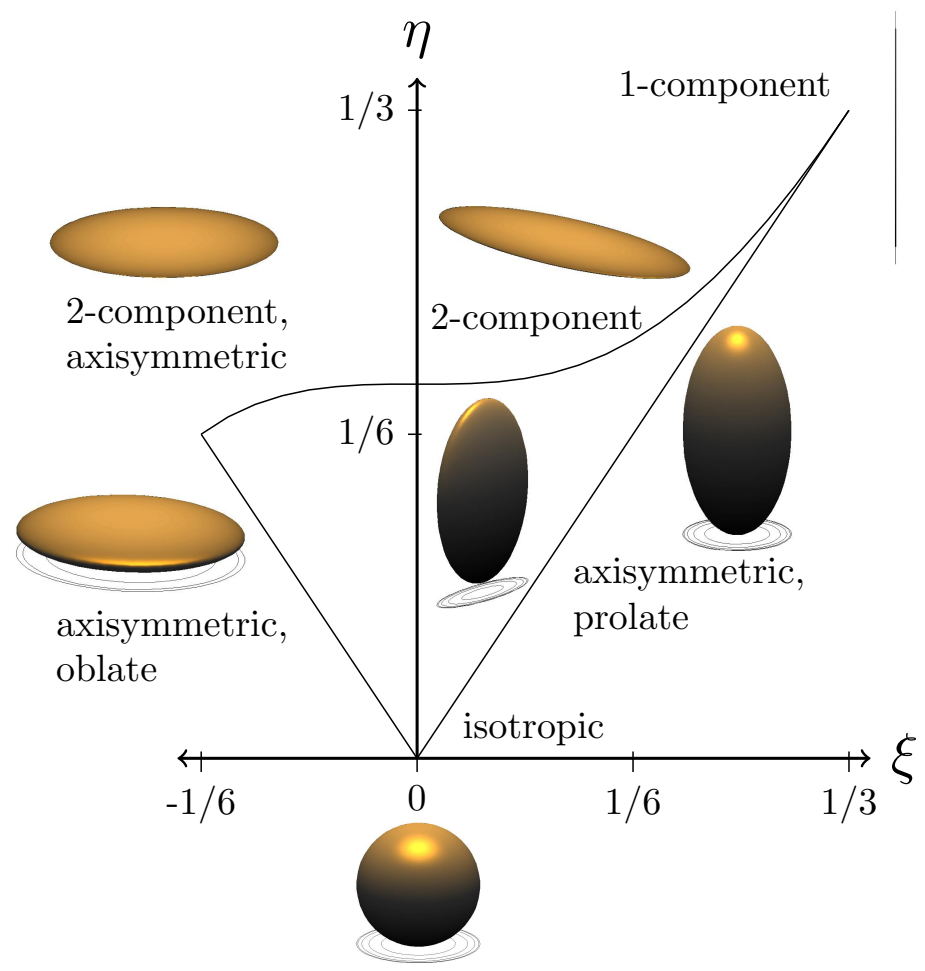

Figure 2.2: Characteristic shapes of $b_{i j}$. Shapes for special cases correspond to those in Table 2.1.

\subsection{Power production of wind turbines}

Power production of wind turbines is the important for wind farm development. The ability to harness kinetic energy from a flow is a function of both the aerodynamic design of the rotor blades and the inflow to the turbine. The power production of model wind turbines will be related to the findings obtained from the theoretical framework 
discussed above. In wind tunnel experiments, the power output of the wind turbines is typically measured as mechanical power,

$$
P_{m e c h}=T_{w t} \omega
$$

in which $T_{w t}$ is the mechanical torque of the turbine rotor and $\omega$ is the angular momentum of the rotor.

As generators are mechanical devices subject to losses of energy, several factors act to significantly decrease the generator efficiency $[3,20]$. These factors include losses in the copper coils via the dissipation of heat in the windings of the generator, magnetic losses in the induction processes converting magnetic fields to electrical current, and mechanical losses from friction between the bearings suspending rotating parts and the bushings or brushes of the generators. Also included in friction-type losses is the air-friction loss of the rotating armature forming the wind turbine rotor. Due to losses of these types, the measured values of electrical power produced by model turbines are expected to be significantly lower than directly measured values of $P_{\text {mech }}$.

The net power available to the wind turbine is commonly defined as,

$$
P_{\text {fluid }}=\frac{1}{2} \rho U^{3} A
$$

where $\rho$ is the density of the air, considered here to be constant, $U$ is the free-stream velocity upstream of the turbine and $A$ is the rotor disc area $A=\pi D^{2} / 4$. $P_{\text {fluid }}$ is derived directly from the conservation of momentum along streamlines in Bernoulli's principle.

The power coefficient of a wind turbine can be computed combining Equations 
(2.12) and (2.13) as,

$$
c_{p}=\frac{P_{\text {mech }}}{P_{\text {fluid }}}=\frac{T_{w t} \omega}{\frac{1}{2} \rho U^{3} A} .
$$

With measurements of mechanical torque $T_{w t}$ and angular velocity, $\omega$, the power coefficient can be calculated when coupled with flow velocity measurements.

The tip speed ratio of wind turbines is a characteristic number with which experiments are commonly scaled and performance of turbines is gauged [1]. The tip speed ratio is commonly defined as,

$$
\lambda_{t s r}=\frac{\frac{D}{2} \omega}{U}
$$

and is the ratio of tangential velocity of the tips of the rotor blades to the mean streamwise velocity across the rotor area. Typical values of tip speed ratios range from $4<\lambda_{t s r}<7$ depending on rotor blade design and particular flow character [26]. 


\section{Chapter 3}

\section{Experimental Setup}

\subsection{Overview of experiment}

The experiment conducted made use of the closed-circuit wind tunnel at Portland State University (PSU). The test section of the wind tunnel measures $5 \times 0.8 \times 1.2 \mathrm{~m}$ in the $x, y$, and $z$ directions, respectively. Figure 3.1 shows the setup for the the current experiment including some typical locations of the velocity measurement. The velocity measurements are made with two-dimensional/three-component $(2 D-3 C)$ stereographic particle image velocimetry (SPIV).

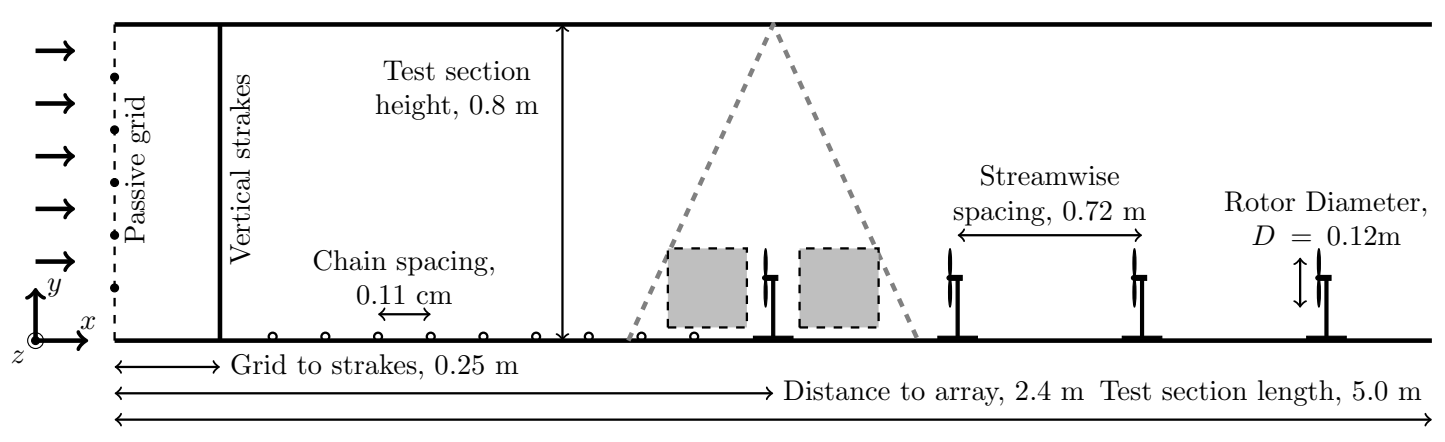

Figure 3.1: Experimental setup shown in wind tunnel test section. Dimensions are for reference only; image not to scale.

The wind tunnel is furnished with an active grid set to passive conditions at the entrance of the test section to introduce turbulence consisting of 7 horizontal and 6 
vertical rods. Removable vertical strakes were fabricated to shape the inflow such that the velocity profile approaching the test section exhibits characteristics of the ABL. The use of vertical strakes is common practice in wind tunnel experiments simulating the atmospheric boundary layer. The strakes were composed of $0.0125 \mathrm{~m}$ thick plexiglass and shaped to extend the shear layer near the wind tunnel floor. There were 9 vertical strakes spaced $0.136 \mathrm{~m}$ apart across the width of the tunnel and placed $0.25 \mathrm{~m}$ downstream of the grid.

As a final conditioning element for the inflow of the experiment, semi-porous surface roughness was added to the floor of the wind tunnel via small-diameter chains. The chains had an average diameter of approximately $0.0075 \mathrm{~m}$ and were spaced 0.11 $\mathrm{m}$ apart extending from the strakes to the first row of wind turbines. The introduction of surface roughness to the boundary layer adds to the influence of the high shear zone near the surface of the wind tunnel.

Figure 3.2 shows characteristic profiles of the inflow parameters as observed by the model wind turbine array. The wind turbine rotors have diameter $D=0.12 \mathrm{~m}$, which is used to normalize spatial coordinates. The mean streamwise velocity profiles in Figure 3.2(A) show changes less than $1 \%$ upstream of the entrance row turbine model.

Figure 3.2(B) shows the turbulence intensities of all three components of velocity in the measurement area. According to boundary layer theory, the turbulence intensity is highest near the wall, which is consistent with the profiles in the figure. For simulated ABL flows, higher turbulence intensity is seen than for flat-plate boundary layers. The turbulence intensity is greatest in the streamwise direction, $\sigma_{u} /|U| \sim 0.2$. In the rotor area $0.5 \leq y / D \leq 1.5$, the streamwise turbulence intensity is on the order of 0.15 , similar to that of previous wind tunnel experiments with turbine arrays $[6,16]$. The vertical 


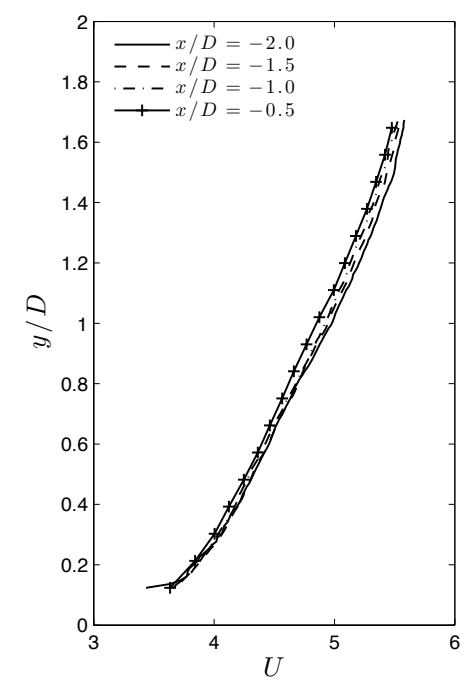

(A) Mean streamwise velocity profiles of inflow boundary layer.

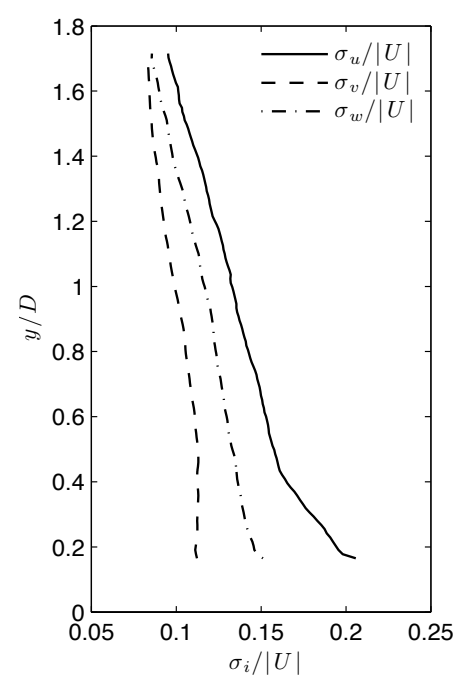

(B) Turbulence intensity profiles of inflow boundary layer.

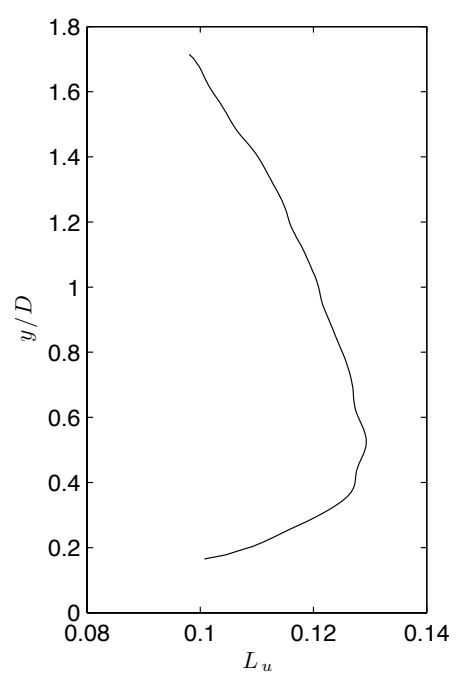

(C) Integral length scales of the streamwise component of velocity.

Figure 3.2: Characteristic quantities pertaining to the approach flow boundary layer of the experiment. All measurements taken upstream of wind turbine array. The streamwise coordinate has been normalized with the turbine rotor diameter.

and spanwise components, $\sigma_{v}$ and $\sigma_{w}$, respectively, are smaller in magnitude than the streamwise component. Figure 3.2(C) shows the profile of the streamwise integral length scale, $L_{u}$, defined as the integral of the auto-correlation coefficient, $\rho(\Delta x)$.

$$
L_{u}=\int_{0}^{\infty} \rho(\Delta x) d \Delta x
$$

where,

$$
\rho(\Delta x)=\frac{\overline{u(x, t) u(x+\Delta x, t)}}{\overline{u^{2}(x, t)}} .
$$

In the rotor area of the approach flow, the integral length scale of streamwise velocity fluctuations is approximately $0.13 \mathrm{~m}$. This length scale is the same order of magnitude 
as the turbine rotor, which is representative of conditions seen by full-scale turbines in atmospheric flows.

Four configurations of a turbine array were tested in the wind tunnel. The primary parameter analyzed in this study is the direction of rotation of the rotor blades. Figure 3.3 shows the arrangements undertaken in the current work. The configuration forming the basis of comparison is that of uniform rotation of turbine blades Figure 3.3(A). Test cases are:

- Row-by-row alternation of rotational sense of the rotors, Figure 3.3(B). The entrance row turbine is identical to that of the uniform case.

- Column-by-column alternation, Figure 3.3(C). The center column of turbines in the array have CCW rotating rotors. Entrance row is identical to that of the checkerboard case.

- Checkerboard case, Figure 3.3(D). Adjacent turbines have opposite rotational direction of turbine blades.

In all cases, the streamwise spacing of turbine rows was held constant at $S_{x}=6 * D$ and the spanwise turbine spacing within the rows was fixed at $S_{z}=3 * D$.

\subsection{Flow measurement system}

The SPIV data in this experiment were collected in two windows simultaneously as shown in Figure 3.4 directly upstream and directly downstream of the entrance and exit row turbines in the arrays, demonstrated by dashed lines in Figure 3.3. A LaVision flow measurement system consisting of an Nd:Yag (532 nm, $1200 \mathrm{~mJ}, 4$ ns duration) pulsed laser and with four CCD cameras (4 MP resolution) arranged in pairs for the 


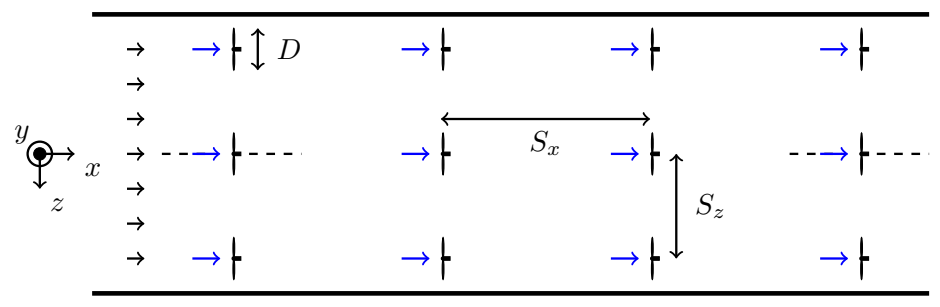

(A) Uniform rotation

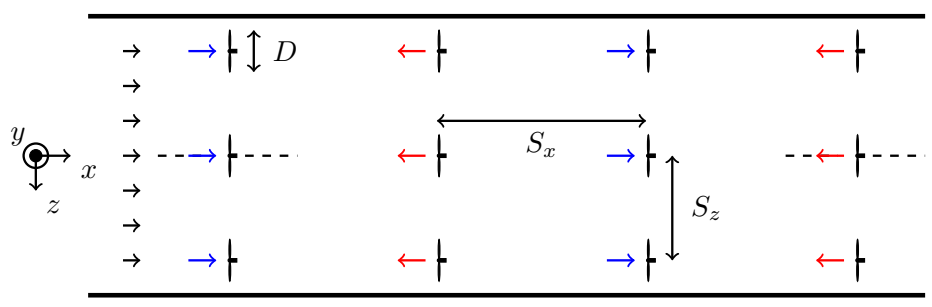

(B) Row-by-row arrangement

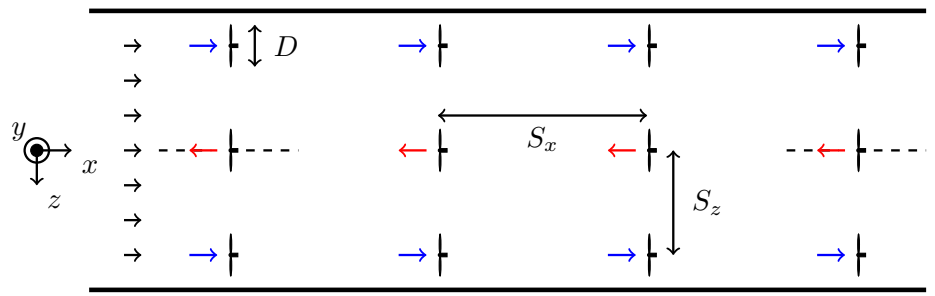

(C) Column-by-column arrangement

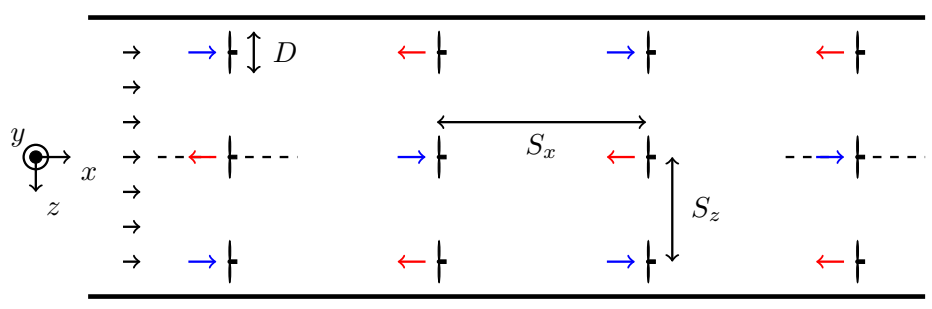

(D) Checkerboard arrangement

Figure 3.3: Configurations of the wind turbine array tested in the PSU wind tunnel. The colored rotation vector indicates rotational sense of the turbine rotors according to the right-hand-rule. Red vectors indicate counter clockwise rotation; blue vectors indicate clockwise rotation. Dashed lines indicate SPIV measurement planes. 
two SPIV interrogation areas. The flow was seeded with neutrally buoyant fluid particles of diethylhexyl sebacate and allowed to mix thoroughly. Seeding was kept at a nearly constant level throughout data collection to ensure resolution of data and mitigate inconsistency in vector calculation. The laser sheet was approximately $0.001 \mathrm{~m}$ thick with a transverse divergence angle of less than 5 mrad across the span of the areas of interest. Measurement locations are shown in Figures 3.1 and a detailed view is seen in Figure 3.4. A single laser sheet was used for both measurement locations and was blocked in the center to reduce reflections from the turbine assemblies.

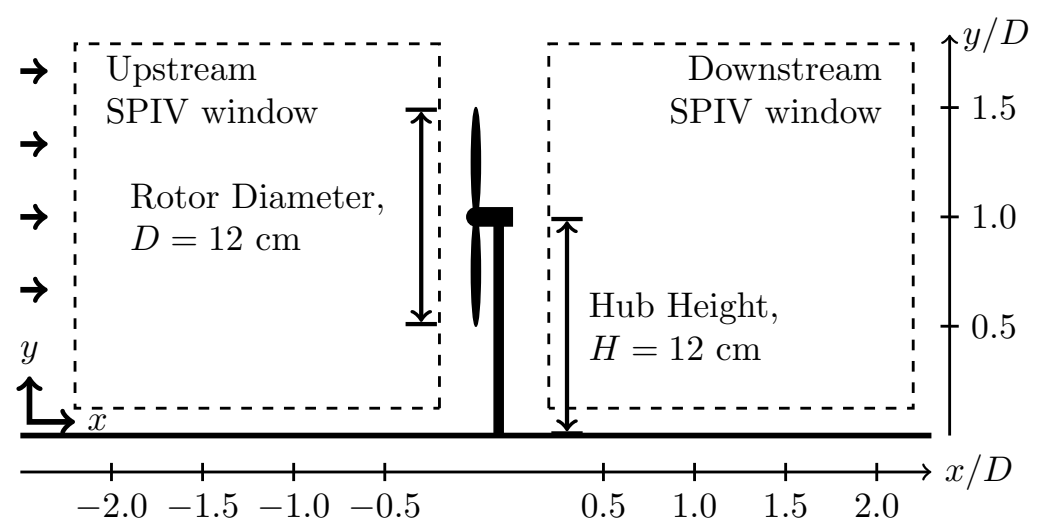

Figure 3.4: Detail view of SPIV measurement areas located directly upstream and downstream of wind turbine models.

Raw images were processed into vector fields using a multi-pass FFT-based correlation algorithm. The interrogation window reduced in size with two passes each at $64 \times 64$ and $32 \times 32$ pixels with a 50\% overlap. The final pass resulted in a final resolution of approximately $1.5 \mathrm{~mm}$ between vectors. The time delay between laser pulses and respective image acquisitions was set to $250 \mu$ s to achieve an average shift of particles of 8 pixels between successive frames. 


\subsection{Wind turbine models}

The wind turbine models were fabricated in-house and consisted of a steel mounting plate $0.01 \mathrm{~m}$ thick, a hollow steel mast, and rotor blades cut from $0.0005 \mathrm{~m}$ sheet steel. The blades of the turbine were given pitch and twist via a press to ensure uniformity. Each blade was pitched approximately $20^{\circ}$ out of the rotor plane at the root of the blade and had a $5^{\circ}$ twist from root to tip, resulting in a pitch of $15^{\circ}$ at the tip of each blade, as seen in Figure 3.5(A). The nacelle of each turbine was a DC electric motor (Faulhaber GMBH \& Co., Series 1331T012SR). Powering the shaft of the motor via the rotors resulted in a measurable current produced across the leads of the motor. The motor was then loaded with resistive elements to slow the rotation of the turbine blades, allowing each row of turbines to be matched to its peak power coefficient according to the work by Delucia [10].

The motors have a cylindrical shell with an outside diameter of $0.013 \mathrm{~m}$ and a nominal operating voltage of $12 \mathrm{~V}$ and a no-load current of $0.0105 \mathrm{~A}$. The motors were aligned with the flow with the shaft pointed upstream. The mast is a hollow cylinder that allowed for electrical wiring for the motor/generator and the torque system to be concealed so that it does not interfere with the flow. A steel mounting plate spanning the test section of the wind tunnel was machined to provide a means of fixing the turbine models in place and orientation. The mounting plate was approximately $0.1 \mathrm{~m}$ wide in the streamwise direction and $0.0075 \mathrm{~m}$ thick. A channel was cut on the underside of the plate to accommodate wiring for the wind turbines and strain gages. The leading edge of the mounting plate was rounded to reduce the introduction of extra turbulence near the wall. Figure 3.5 shows schematics of the fully assembled turbine models including mast, nacelle, rotor, mounting plate, and a detail view of the rotor 
before blade twisting.

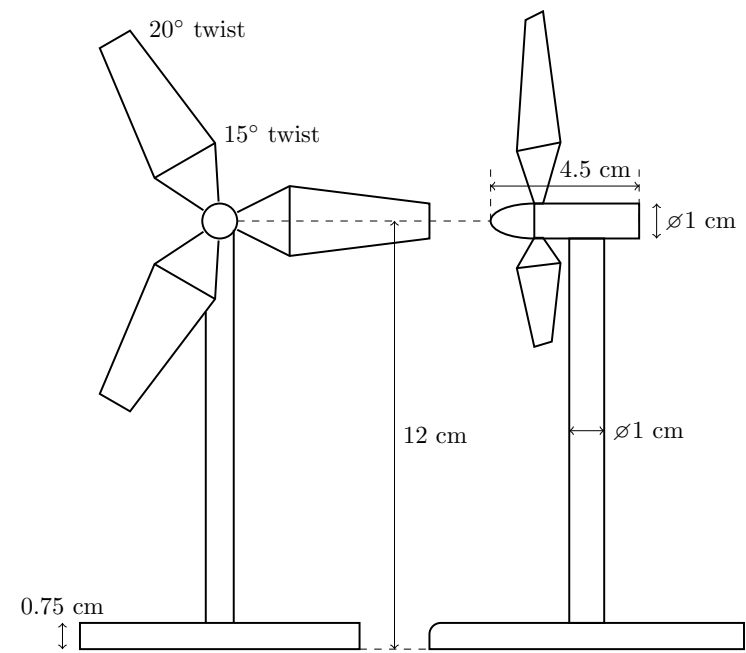

(A) Perspective views of wind turbine models used in experiments.

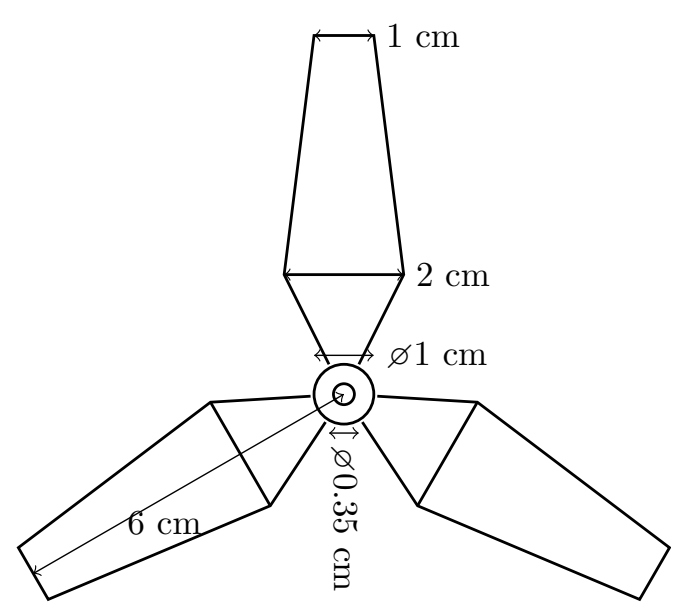

(B) Detail of rotor model before addition of blade twist.

Figure 3.5: Technical drawing of model wind turbines used in experiments. All dimensions are in millimeters. The mounting plate shown in 3.5(A) spans the full width of the wind tunnel; only a small section is shown.

\subsection{Torque sensing system}

The torque sensing system implemented in the experiment followed the design and procedure outlined by Kang and Meneveau [20] and employed in the PSU wind tunnel by Hamilton et al. [16]. The motor was supported within a cylindrical metal housing by two VXB ball bearings aligned concentrically with the shaft of the generator. The inner and outer diameters of the bearings were $0.013 \mathrm{~m}$ and $0.024 \mathrm{~m}$ respectively, and the outer diameter of the housing was approximately $0.0254 \mathrm{~m}$. The bearings allowed the entire generator to rotate in reaction to the application of torque on the shaft. On the downstream face of the motor/bearing housing a pin was set such that application of torque resulted in the flexure of a bending arm made of a strip of $0.0005 \mathrm{~m}$ thick 
bronze, $0.005 \mathrm{~m}$ by $0.02 \mathrm{~m}$ on a face. To the bronze strip were mounted two $120 \Omega$ linear strain gauges (OMEGA SGD-3/120-LY11), measuring the torque required to keep the motor stationary relative to the tunnel. Figure 3.6(A) shows the assembly of the motor/bearing housing and component elements.

The signals from the strain gauges on the bending arm were measured with a Wheatstone bridge constructed with two additional $120 \Omega$ strain gages. The additional gages were mounted to an aluminum cube measuring $0.1 \mathrm{~m}$ per edge to provide a constant reference signal. The entire strain-gage/bridge system was conditioned and amplified by an external electrical module (OMEGA DMD-465 Bridgesensor) and measured at $10 \mathrm{kHz}$ with LabVIEW and a National Instruments DAQ.

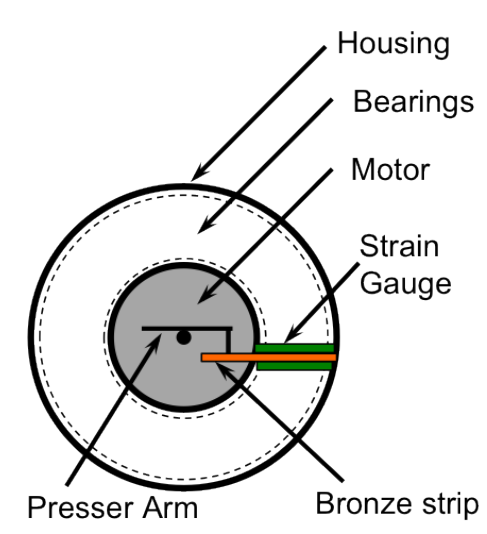

(A) Torque sensor showing key components.

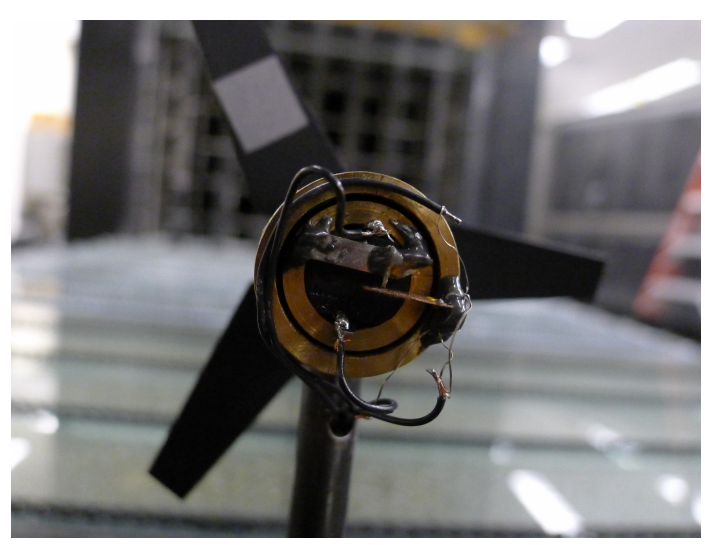

(B) Torque sensor mounted to a wind turbine model and placed in the wind tunnel.

Figure 3.6: Torque sensor used in the experiment. Reflective tape show on the topmost rotor blade in 3.6(B) was used in the measurements of angular velocity by the remote optical sensor.

Rotor blade tip speeds were monitored through a remote optical sensor (Monarch ROS) and controlled by applying resistive loads to the motors. During velocity measurements, each row of turbine models was set to operate under loading conditions 
corresponding to the peaks of their respective power curves. The hollow masts allowed electrical wiring for speed control and strain measurements to be contained and out of the mean flow mitigating added effects in the main wake area. 


\section{Chapter 4}

\section{Results}

\subsection{Mean flow statistics}

The SPIV measurements taken directly upstream and downstream of the entrance and exit row turbine models are reviewed in the following figures. Much of the discussion below is focussed on the turbulence and behavior of exit row wind turbines. For the mean velocities, contour plots follow the layout described in Figure 4.1 wherein the left pair of columns correspond to the inflow and outflow of the entrance row turbine position and the right pair of columns correspond to exit row turbines. Rotational sense of the turbine models is indicated in the diagram.

The normalized mean streamwise velocity $U / U_{\text {hub }}$ in all measurement locations is shown in Figure 4.2, where $U_{\text {hub }}=4.75 \mathrm{~ms}^{-1}$. The first column of contour plots correspond to the approach flow to the arrays and match well upstream $(x / D \leq-1.5)$ of the model array. The wakes of the entrance row turbines, however, demonstrate some difference based on particular array configuration. The wake of the counter-clockwise $(\mathrm{CCW})$ rotating blades in the checkerboard case shows a greater momentum deficit $p$ in the near wake than does the clockwise $(\mathrm{CW})$ rotor found in the uniform case. The momentum deficit is defined as, 


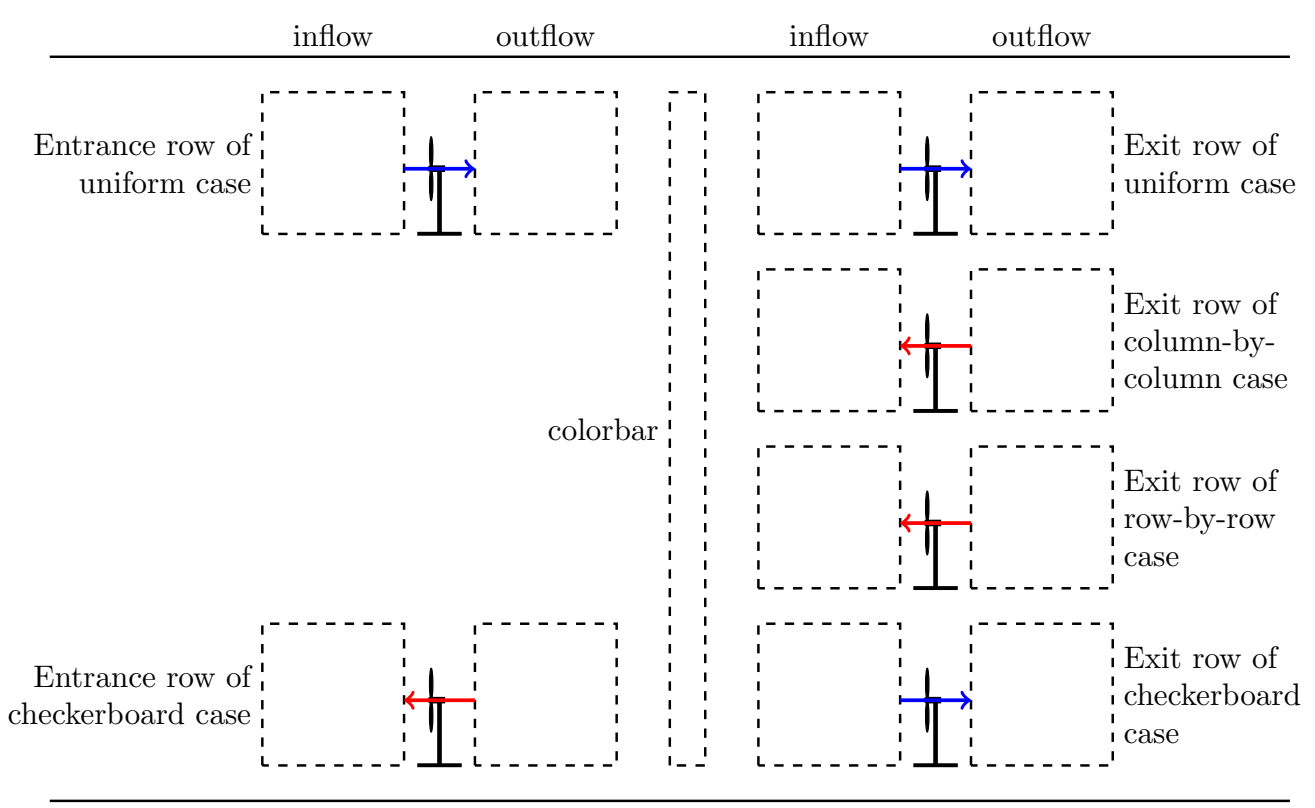

Figure 4.1: Positions of contour plots of mean velocities, $U$ and $W$ in Figures 4.2 and 4.3.

$$
p=\frac{1}{A} \int_{A} \frac{U}{U_{\text {approach }}}\left(1-\frac{U}{U_{\text {approach }}}\right) d A,
$$

where $A$ is the measurement area and $U_{\text {approach }}$ is the mean velocity profile seen in the approach flow. For the uniform case, $p \approx 34 \%$, whereas the checkerboard configuration shows, $p \approx 50 \%$ of the mean momentum as compared to the approach flow.

The inflows to exit row turbines show momentum deficits of $p \approx 30 \%$ within the turbine array. These windows are also representative of the far wakes of the third row of turbines and show $0.6 \leq U / U_{\text {hub }} \leq 0.8$. The blockage effect of the exit row of turbines is seen from $x / D>-0.75$. The inflows for the uniform and checkerboard cases show magnitudes in streamwise velocity approximately $7 \%$ larger than the row and column cases.

The mean wall-normal velocity, $V / U_{\text {hub }}$ (not shown for brevity), featured consis- 
tent trends in all measurement locations regardless of row position or rotational sense of the turbine rotor. Extreme magnitudes of $V / U_{\text {hub }}$ are approximately $5 \%$ larger for entrance row turbines than for those in the exit row. The vertical velocity in inflow measurement windows reflects the blockage seen in Figure 4.2 as flow around the turbine rotor, upward above the nacelle and downward below. In each of the wakes there is a mean vertical velocity into the wake. This serves to confirm the resupply of momentum through the flux of kinetic energy into the rotor area, discussed further below.
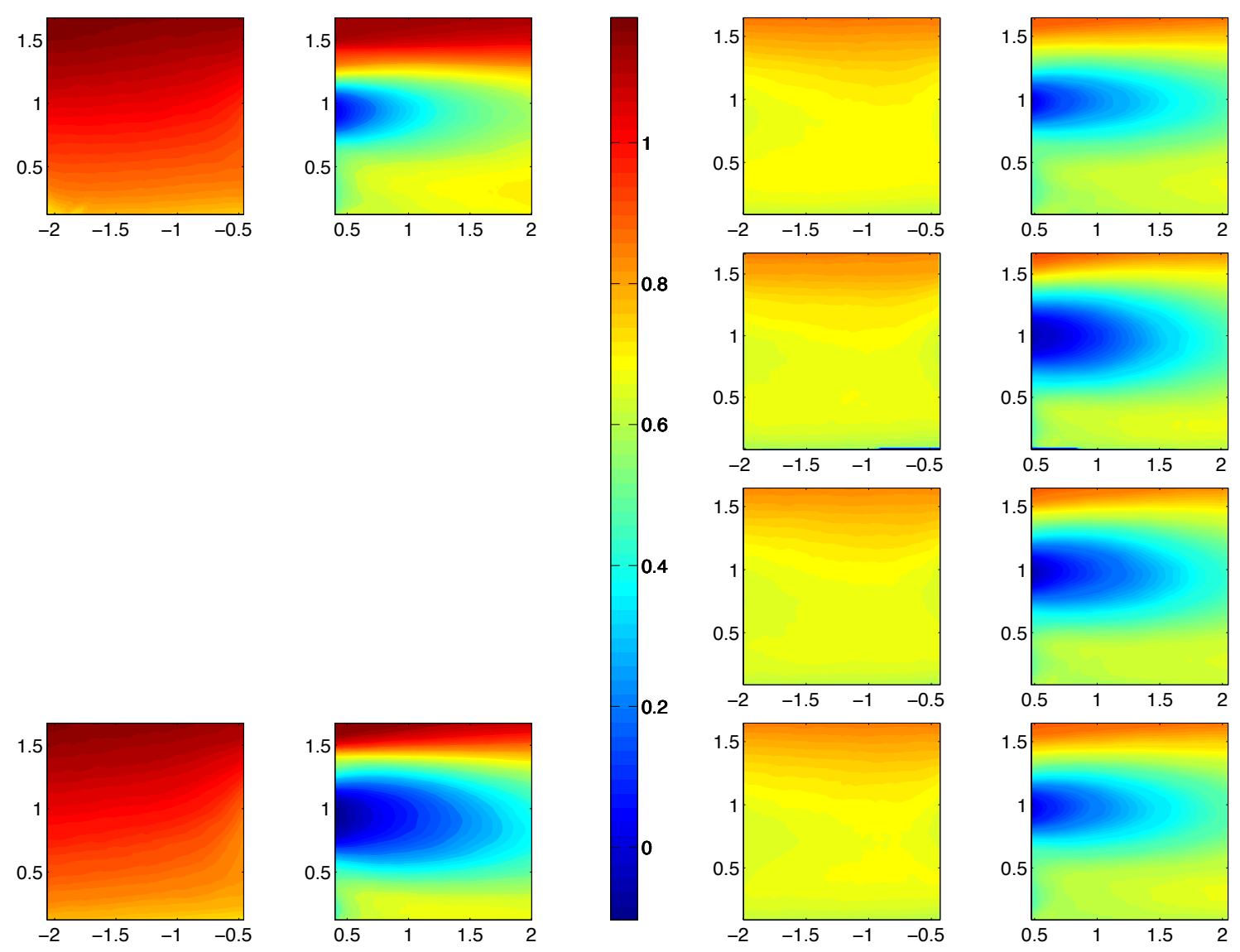

Figure 4.2: Mean streamwise velocity normalized by the inflow velocity at hub height, $U / U_{\text {hub }}$.

The normalized spanwise velocity $W / U_{\text {hub }}$ (out-of-plane) shows the rotational 
sense of the turbine rotors clearly, illustrated in Figure 4.3. Turbines with CW blades (top row and bottom right) show a positive spanwise velocity (toward the reader) associated with the top rotor tip and a negative spanwise velocity associated with the bottom rotor tip. The opposite is seen for the CCW blades. For turbines in the entrance row there is also a positive spanwise velocity associated with the mast of the turbine model regardless of direction of rotation. Another distinction between the CCW and CW turbine rotors in the entrance row is that the magnitude of the spanwise velocity is greater in the uniform case and weaker in the checkerboard case. This trend is opposite from that of the normalized streamwise velocity.

The exit row of turbines show a similar order of magnitudes for both top and bottom tip areas regardless of direction of rotation. The mean spanwise velocity in the area directly behind the masts of the turbine models is negative, opposite of the entrance rows. In the exit row wakes, the negative spanwise velocity is nearly double $\left(W / U_{\text {hub }} \approx-0.15\right)$ that of the entrance rows. The regions of distinct spanwise velocity also extend farther into the wake suggesting that turbines in the fully developed region of the array impart a greater mean out-of-plane motion than in leading rows.

Mean velocities provide an overall sense of the flow and the behavior of the wind turbines within the arrays but exclude most of the effects of turbulence within the turbine canopy layer. In order to investigate the terms on the right side of Equation (2.2), it is necessary to investigate the Reynolds stresses. The turbulent kinetic energy $k$ is consistent regardless of rotational sense of rotors but shows distinctively different behavior in wakes created by turbines further from the entrance row of the array. As the turbulent kinetic energy is comprised of only the Reynolds normal stresses, it provides information regarding only stretching or compressing stresses. 

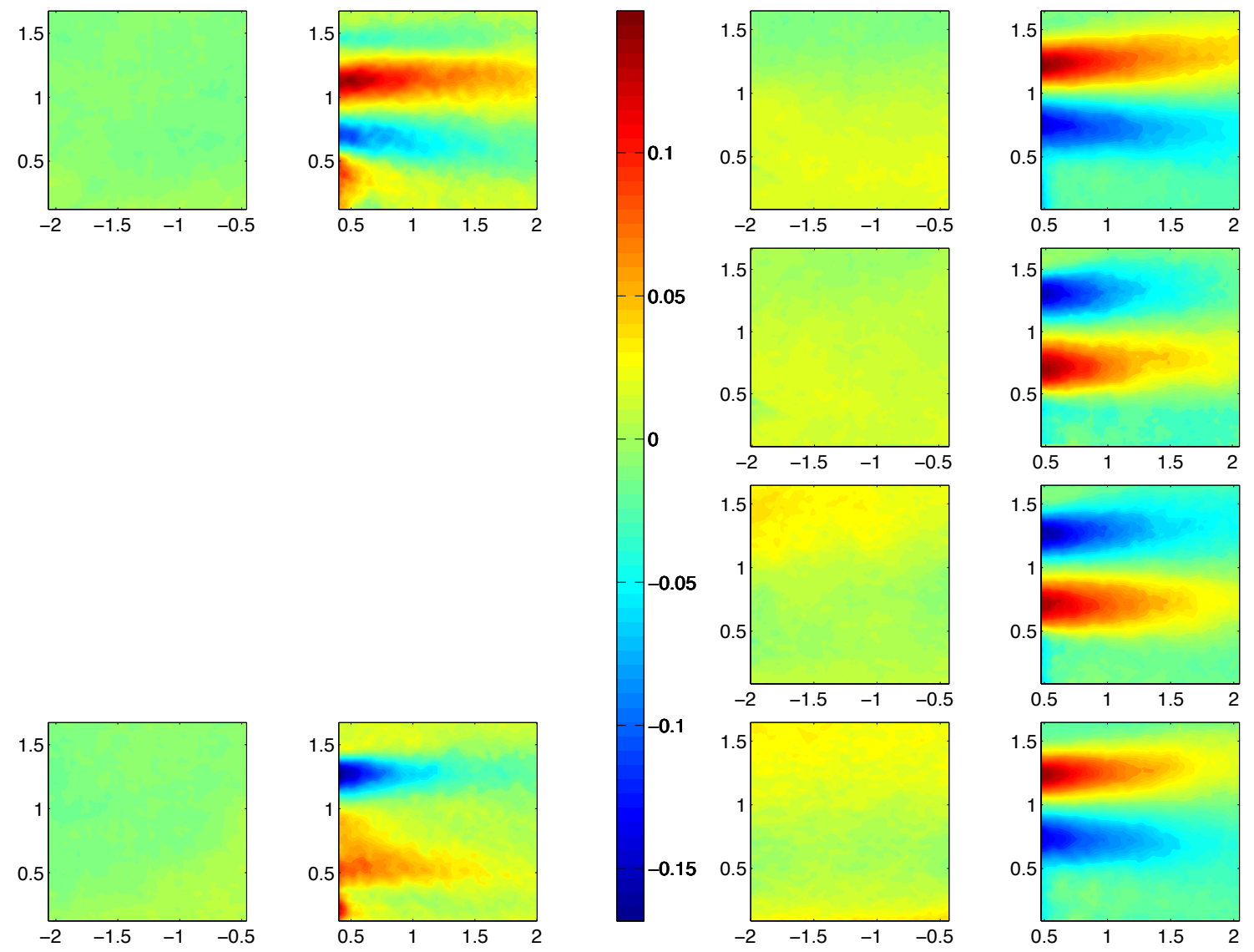

Figure 4.3: Mean spanwise velocity normalized by the inflow velocity at hub height, $W / U_{\text {hub }}$.

Shear stresses in the mean mechanical energy equation are of importance in the full energy budget, especially when considering the flux and production terms. Apparent shear stresses in the tensor $\overline{u_{i} u_{j}}$ indicate that the stress tensor is not in its principle form. Two of the Reynolds shear stresses are shown in Figures 4.4 and 4.5. The stress $-\overline{u w}$ is not used in the components of the flux or production tensors discussed here. It does not demonstrate significant changes between cases.

The normalized in-plane shear stress $-\overline{u v} / U_{\text {hub }}^{2}$ in Figure 4.4 shows fairly consistent trends for each of the wakes of exit row turbines. There is an increase in magnitude 
$(\sim 15 \%)$ for turbines rotating in the CCW sense. These differences are more clearly seen in the region of the wake following the top rotor tip. The sign of $-\overline{u v} / U_{\text {hub }}^{2}$ shows no dependence on rotational direction of the turbine because the measurements are in the central plane of the rotor, where fluctuations in both the streamwise and wall-normal velocities demonstrate near-symmetry in the spanwise sense. The intensities of the shear stresses correlate well with gradients in the mean flow between regions of the wakes and the canopy (Figure 4.2). The inflow to fourth row turbines is very similar in all cases $\left(-2 \times 10^{-3} \leq-\overline{u v} / U_{\text {hub }}^{2} \leq 7 \times 10^{-3}\right)$, regardless of rotational sense of upstream turbines.

Rotational sense stands out in the shear stress $-\overline{v w} / U_{\mathrm{hub}}^{2}$. Figure 4.5 indicates that in the exit row of turbines, the inflow is nearly uniform and null. In the wake area, rotors with a CW orientation demonstrate negative values associated with the top and bottom rotor areas. The opposite is seen for turbines with a CCW rotational sense, although magnitudes are nearly equal. The shear stress $-\overline{v w} / U_{\text {hub }}^{2}$ is short-lived compared to the in-plane shear stress. The mean flow imparts motion to the rotor blades as shown in the diagram on the right side of Figure 4.5. At the same time, the rotor blades exert force on the flow in the opposite direction, imparting dependence of rotation of the blades in the velocity fluctuations. Spanwise fluctuations have the same algebraic sign of those seen in the vertical velocity for the row-by-row and column-bycolumn cases (CCW blade rotation), resulting in positive shear stresses in the wake. The fluctuations have opposite algebraic signs in the cases of uniform and checkerboard rotational schemes, resulting in negative shear stresses for these cases. When the rotor blade is moving in the CW sense, the regions of high stress associated with the top and bottom rotor tips extend farther in the streamwise direction as compared 


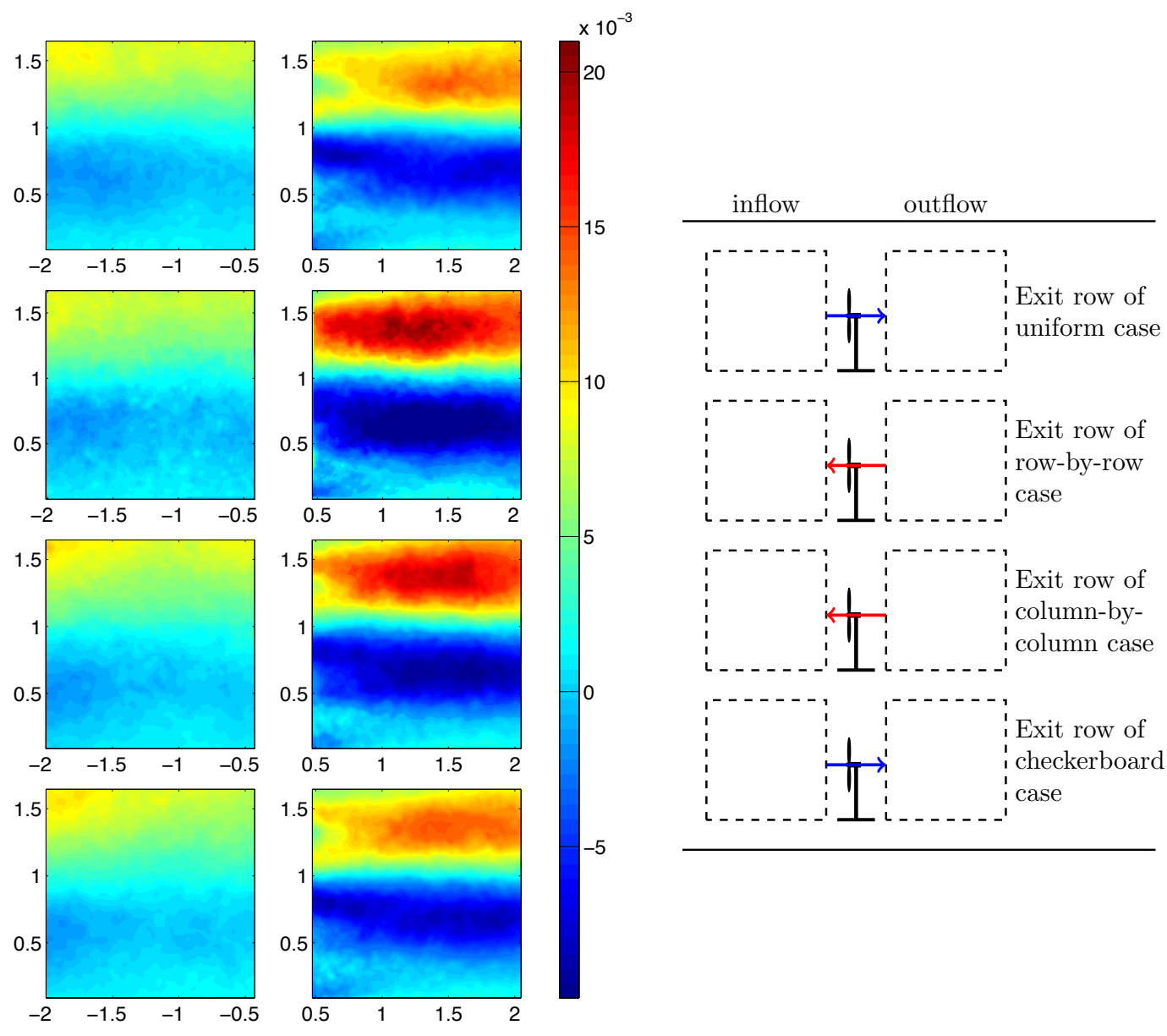

Figure 4.4: Normalized Reynolds shear stress $-\overline{u v} / U_{\text {hub }}^{2}$.

to those of CCW blades. This indicates that $-\overline{v w} / U_{\text {hub }}^{2}$ effects the stress balance for more of the wake for those cases.

The flux of turbulence kinetic energy in the wakes of wind turbines is responsible for the resupply of momentum into the turbine rotor area of the canopy. This has been shown in other wind turbine studies [4, 15-17] to be one of the primary mechanisms responsible for the functioning of turbines in the fully developed region of the infinite wind farm. The in-plane component of the flux tensor $F_{12}=\overline{u v} U$ has been demonstrated as the primary contributor to the overall flux and is shown in Figure 4.6. 


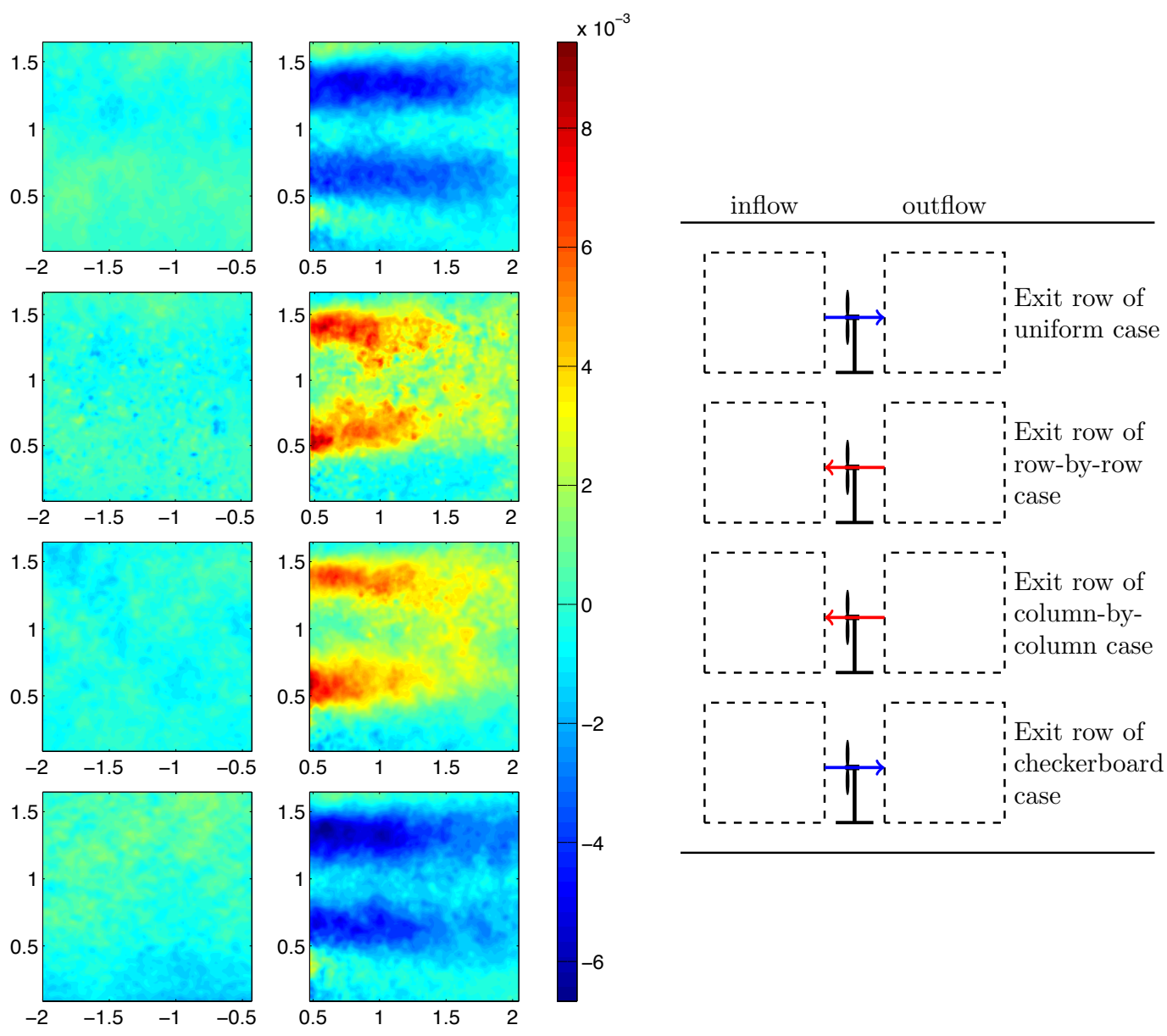

Figure 4.5: Normalized Reynolds shear stress $-\overline{v w} / U_{\mathrm{hub}}^{2}$.

The flux of kinetic energy is a point-wise multiplication of the in-plane Reynolds stress $-\overline{u v}$ and the mean streamwise velocity $U$. Due to its composition, the regions of greatest flux into the wake are associated with the top rotor tip where there are significant stresses and mean velocity. This is shown to be true especially in the CCW blades as they demonstrate increased turbulence activity in the wakes. The maximum values of $F_{12}$ are approximately $1.3 \mathrm{~m}^{3} / \mathrm{s}^{3}$ occurring approximately $x / D=1.5$ into the wake.

A positive flux term implies entrainment of high-momentum flow downward from above $[4,17]$. Similarly, a negative value of flux indicates entrainment from below the 
rotor area upward. The flow below the rotors is constrained by the floor of the wind tunnel and experiences the development of a boundary layer between each row of wind turbines. This effectively limits the momentum of the flow available for upward flux into the wake to approximately $1 / 3$ of that of the upper canopy. This trend is seen in all cases for $y / D \geq 1$. The contours in Figure 4.6 indicate that peak flux values occur within the near wake but extend into the far wake, as evident in the inflow measurements. The flux of kinetic energy is reflected by the mean vertical velocity in which there is a mean flow toward the center of the wake from the edges.
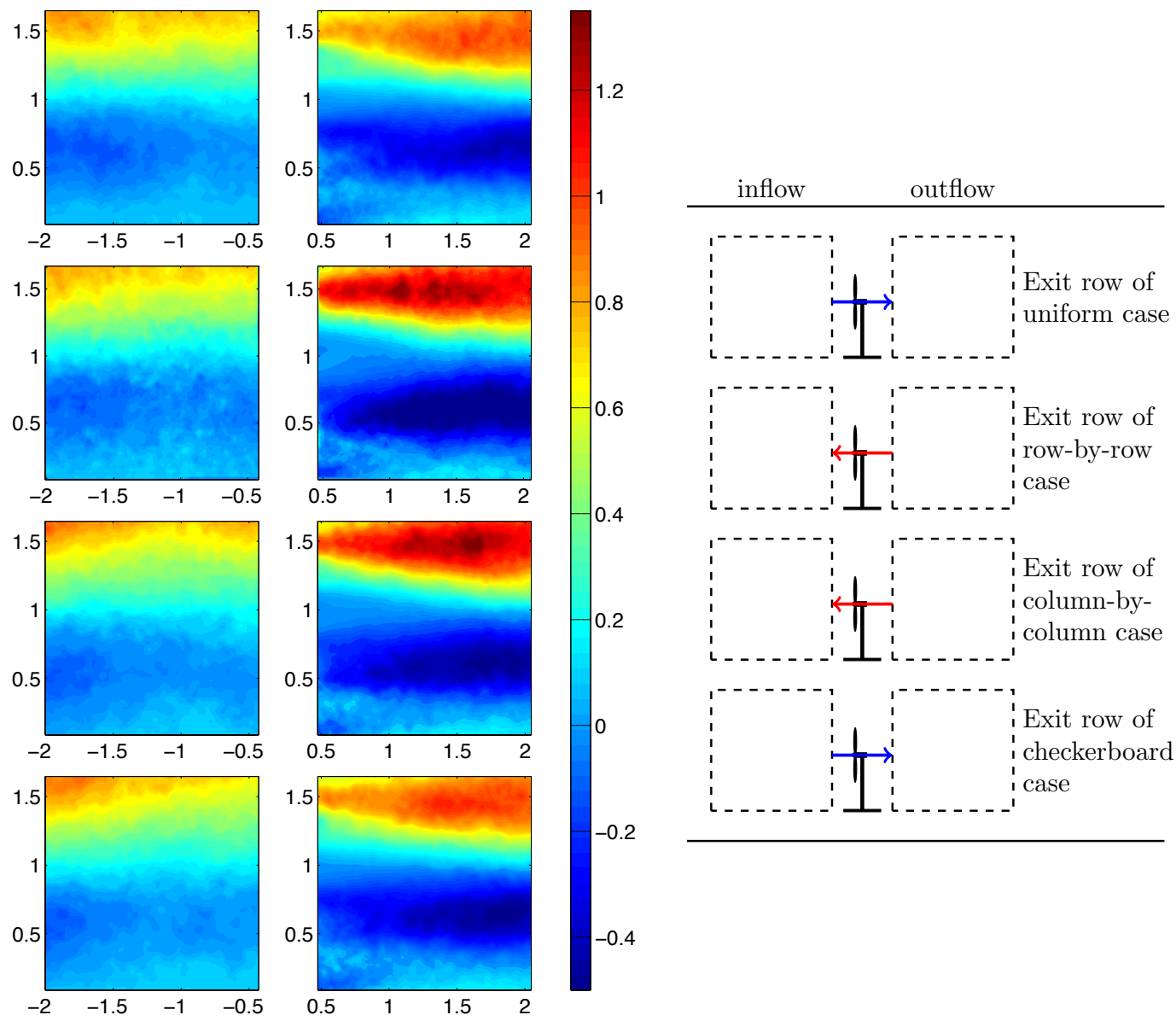

Figure 4.6: In-plane flux of turbulence kinetic energy, $F_{12}=-\overline{u v} U$. 
The production of turbulence kinetic energy $P_{i j}$ makes a smaller contribution to the overall energy balance suggested by Equation (2.2) than the flux of turbulence kinetic energy but is included for its theoretical interest. The production of turbulence kinetic energy is again a second order tensor whose elements vary in magnitude depending on specific flow geometry. As with the flux tensor, only the in-plane component of production $P_{12}=\overline{u v} \frac{\partial U}{\partial y}$ is included here as it represents the greatest contribution to the tensor, approximately $65 \%$ of the production described by the components available with the current measurements.
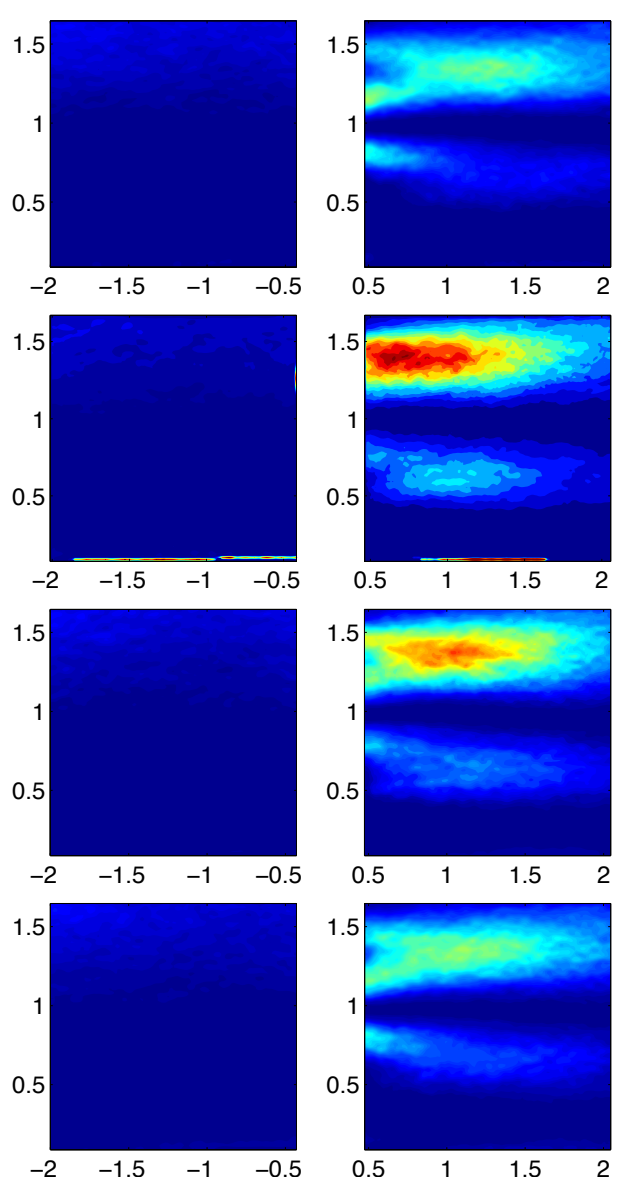

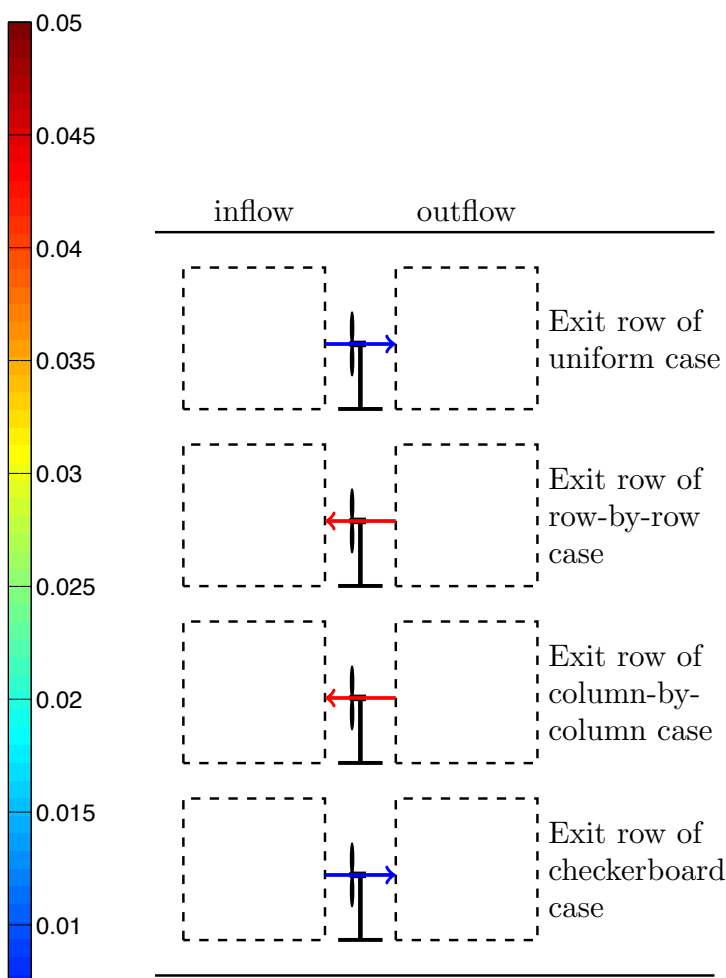

0.005

Figure 4.7: In-plane production of turbulence kinetic energy, $P_{12}=-\overline{u v} \frac{\partial U}{\partial y}$. 
The composition of $P_{12}$ requires that both the in-plane Reynolds stress and a wallnormal gradient of the mean streamwise velocity be present to generate non-null turbulence kinetic energy production. This is shown in the contour plots of Figure 4.7. The characteristic shape of the mean in-plane Reynolds stress contours are evidenced in the wakes areas, especially near the top rotor tip. There is a lesser contribution to the production of turbulence kinetic energy following the lower half of the rotor areas. This contribution is lower due to the softened gradients of the streamwise velocity in that area.

The wake of the row-by-row configuration shows peak values of $P_{12}$ on the order of $0.05 \mathrm{~m}^{2} / \mathrm{s}^{3}$ approximately twice that of the uniform and checkerboard cases. Peak values occur within one rotor diameter of the top tips of the rotor blades in all cases. The difference in peak values of $P_{12}$ is attributed to a difference in $-\overline{u v}$ between cases. The gradient $\frac{\partial U}{\partial y}$ changes less than $10 \%$ between array configurations and cannot account for the difference in production by itself. The production following the bottom rotor blade is approximately $0.015 \mathrm{~m}^{2} / \mathrm{s}^{3}$ for all cases.

Discussed in $[4,16]$, the production of turbulence kinetic energy has another meaning when viewed from the perspective of a wind turbine. The production term quantifies how much mean kinetic energy is converted into turbulence by shear stresses and mean flow gradients. Wind turbines convert mean flow energy into mechanical energy and can convert little turbulence energy. That said, the production term can be viewed as the amount of mean flow energy dissipated into turbulence and made unavailable to successive devices in the array. 


\subsection{Analysis of anisotropy}

As mentioned in the discussion of the turbulent kinetic energy above, having turbulent shear stresses that are of similar order of magnitude as normal stresses indicates that the stress tensor is not in its principle axes. This suggests that $k$ may not capture or communicate the full energy of turbulence present within a flow. Further, it indicates that there may be a more efficient coordinate system for describing the turbulent stresses. The normalized Reynolds stress anisotropy tensor $b_{i j}$ is decomposed in order to obtain the eigenvalues according to Equation (2.10). This decomposition occurs in a point-wise sense such that the invariants $\eta$ and $\xi$ (Equation (2.11)) are realized in the full measurement fields. Contour plots of the invariants of $b_{i j}$ are shown in Figures 4.8 and 4.9 for the exit row turbine of each array configuration.

Looking to the special states of turbulence described by the anisotropy invariant map in Figure 2.1, $\eta$ is more closely related to the degree of anisotropy than it is to the characteristic shape of the anisotropy tensor. Based on this observation, several conclusions can be drawn regarding the contour plots of $\eta$ seen in Figure 4.8. The most prevalent trend seen in the figure is that there is a region of large $\eta$ trailing the top rotor tip in each case. This indicates that the turbulence in the upper region of the turbine rotor canopy is the most anisotropic seen in the wake. The minimum values of $\eta$ occur following the nacelle. This is also the region, generally speaking, where shear stresses are at a minimum for each of the wakes, implying that the turbulence stress tensor may be near its principle condition. This behavior is attributed to the return to isotropy demonstrated by decaying turbulence.

According to the inflows to the exit row turbines, the region of small $\eta$ grows as one looks downstream of the turbines, indicating that the cores of the wakes are fairly 

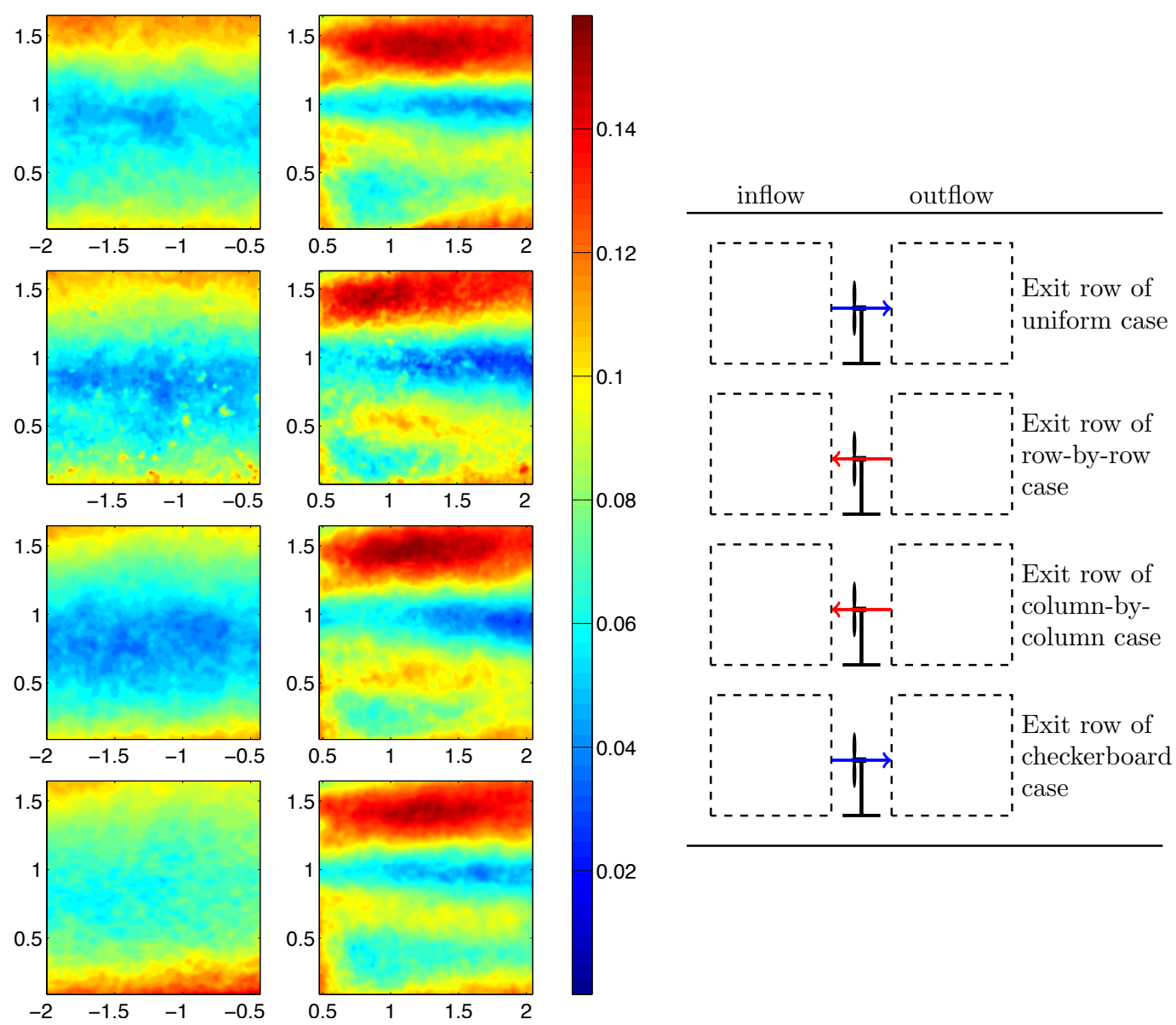

Figure 4.8: Plots of second invariant of the normalized anisotropy tensor, $\eta$ corresponding to the ordinate of the Lumley triangle. The degree of anisotropy is associated with $\eta$.

isotropic and that the region of relative isotropy grows outward from the hub progressing with the main flow. In the inflow of all cases, there is a region of relatively large $\eta$ near the wind tunnel floor and above the rotor area, but the degree of anisotropy is not uniform from case to case. According to the contour plots in Figure 4.8, inflows to the CW rotating blades are generally more anisotropic than the CCW blades, especially in the rotor area. This is seen especially in the inflow to the exit row turbine in the checkerboard case, where the minimum value of $\eta$ is approximately 0.05 , as opposed 
to the column-by-column case, where $\eta$ reaches a minimum value of approximately 0.03 in its inflow. Regardless of configuration, the minimum value of $\eta$ is still aligned with the hub in the far wake of the turbine.

The turbines with a lesser magnitude of $P_{12}$ (uniform and checkerboard cases, CW blades) show greater values of $\eta$ in their inflows. This is suggests that when a wind turbine experiences less isotropic turbulence in its inflow, the production of turbulence kinetic energy is reduced in its wake. The connection between the production and the degree of anisotropy is discussed more in Section 4.4. According to the Lumley triangle shown in Figure 2.1, truly isotropic turbulence, which requires $\eta=0$ is not reached in any of the cases.

The third invariant of $b_{i j}$ is shown in Figure 4.9. The wake areas evidence negative values of $\xi$ following the nacelle and positive regions following the tips of the rotors. The regions outside of the rotor area ubiquitously demonstrate $\xi>0$. From the Lumley triangle in Figure 2.1, it is evident that the form of the characteristic spheroid is well described by $\xi$. Positive values of $\xi$ lead to a stretched, or prolate, spheroid and negative values lead to a squeezed, or oblate, shape. From the wakes shown in Figure 4.9, the turbulence following the extremes of the rotors tends toward prolate spheroids and the characteristic spheroids following the nacelles of the turbines are dominantly oblate.

Another point of interest in the figure is that the $\xi<0$ region of the wakes drifts slightly upward or downward depending on the direction of rotation of the rotors. The uniform and checkerboard cases show a general downward drift progressing downstream of the turbines. The CCW rotors show a slight upward drift of this region. A common feature of all measurement locations is that $\xi$ is always either significantly positive or negative in the near wake. This reinforces the point made above that the 

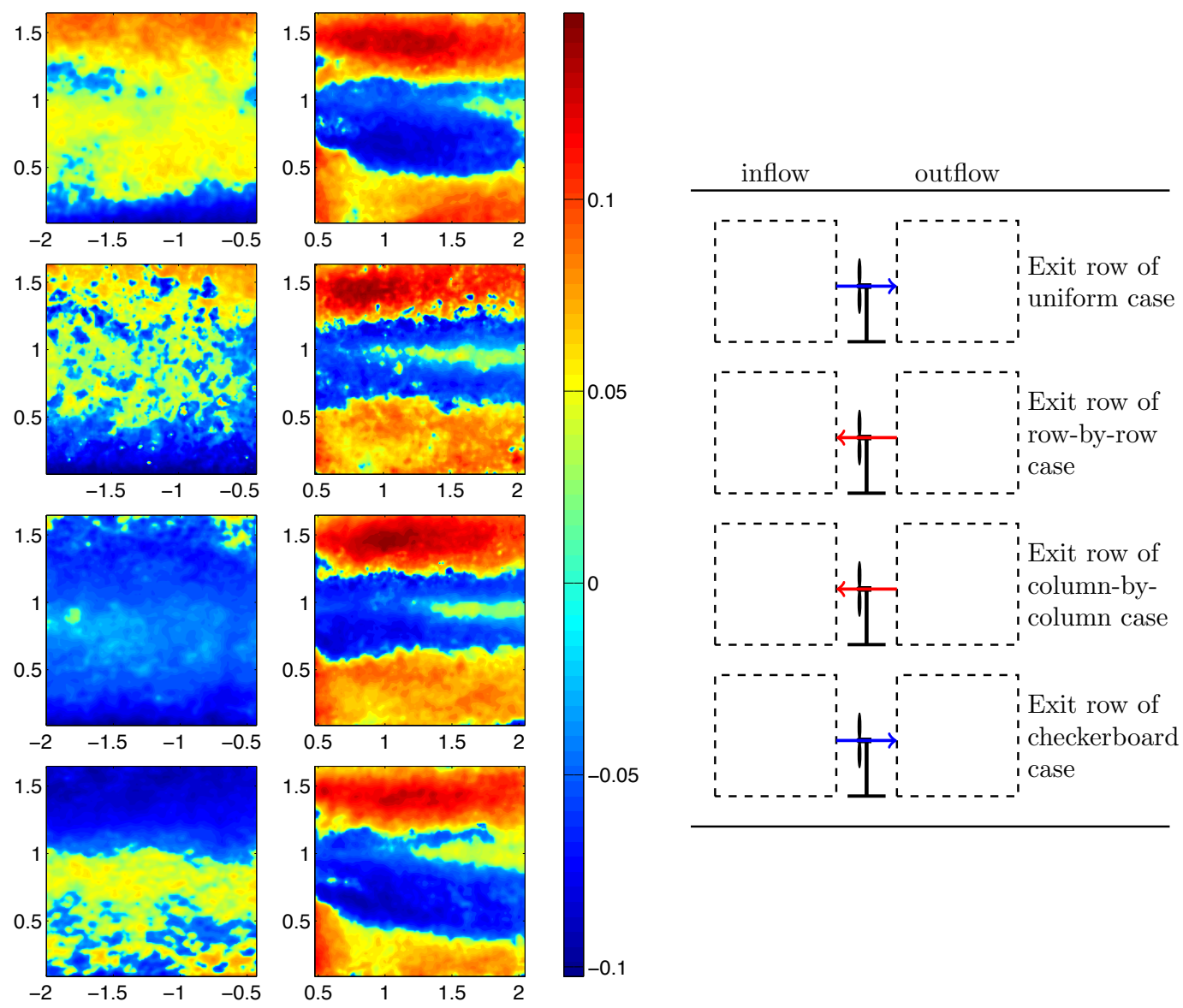

Figure 4.9: Plots of third invariant of the normalized anisotropy tensor, $\xi$ corresponding to the abscissa of the Lumley triangle. Characteristic shapes of Reynolds stress anisotropy are described by $\xi$.

turbulence never reaches a truly isotropic state close to the wind turbines, where $\xi=0$ (and $\eta=0$ ) would be required. However, looking to the inflow windows $\xi$ approaches zero in all cases. This implies a general return to isotropy in the far wakes $(x / D>4)$ of third row turbines. That the invariant is never identically zero is likely an artifact of the numerical decomposition.

The invariants shown in both Figures 4.8 and 4.9 can be mapped against one another in the Lumley triangle. As discussed in Table 2.1, special cases of turbulence can 
be represented by the two invariants of $b_{i j}$ and the theoretical limits of the Lumley triangle. Although the contours above reveal that the turbulence never reaches a truly isotropic state in the measurement domain, the trends suggest that turbulence is organized into distinct regions within the wake as the flow moves away from the turbines.

Data from the measurement locations is plotted against the Lumley triangle in Figure 4.10. In each of the anisotropy invariant maps in the figure, invariants corresponding to the upstream window, represented by black points, $\eta$ and $\xi$ are more tightly grouped than those from the downstream measurement window, represented by red points. This indicates that the anisotropy of turbulence is more uniform in the far wakes of the wind turbines. The figure also demonstrates that there are both prolate and oblate characteristic spheroids in all measurement cases. The exception to this trend is seen for the flow directly upstream of the column-by-column case. There the characteristic shapes overwhelmingly favor oblate spheroids. This effect is due to the counter-rotating columns of turbine rotors generating large-scale streamwise structures according to the rotational sense of the rotors. In the wake area of the same case, the turbulence favors prolate spheroids, as in the other cases, but show slightly higher value of $\eta$, indicating a larger degree of stretching than in the other wakes.

The inflows to exit row turbines include cumulative wake effects of upstream turbines in their own columns as well as those from neighboring columns. The complementary rotation of columns of turbines in the column-by-column case create the effect seen in the inflow window of Figure 4.10(C). The inflow to the exit row of the checkerboard case (Figure 4.10(D)) shows less consistent trends of $\eta$ than other cases. Mentioned above, a greater magnitude of this invariant implies less isotropic turbulence. This indicates that the far wakes of turbines in the checkerboard arrangement 


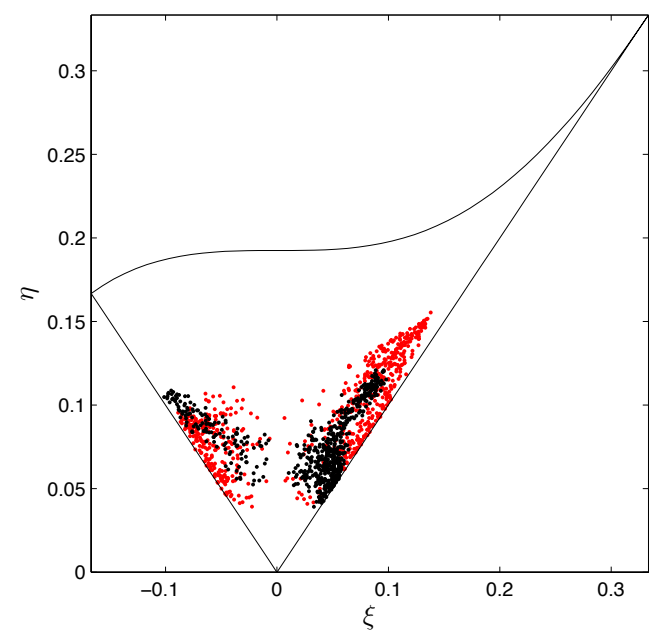

(A) Exit row of the uniform arrangement

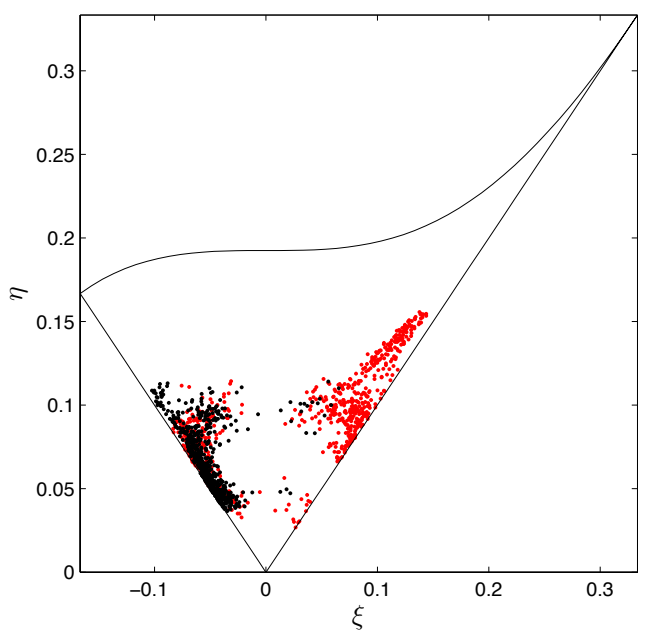

(C) Exit row of the column-by-column arrangement

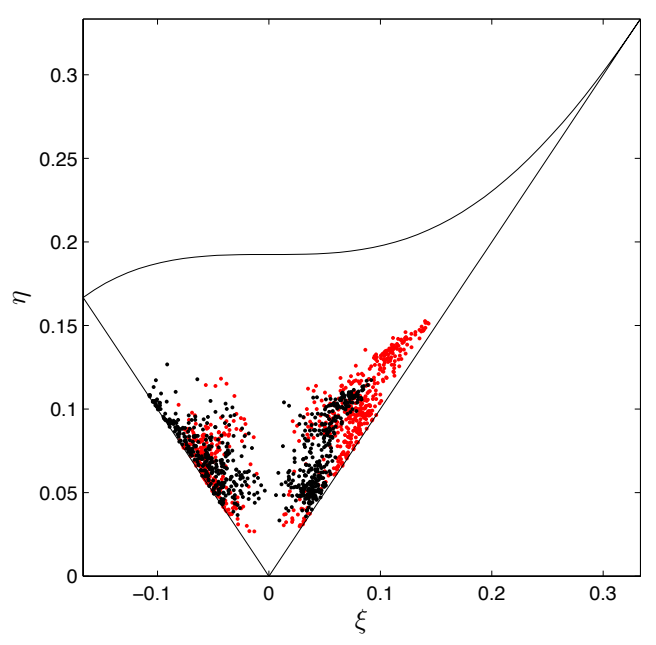

(B) Exit row of the row-by-row arrangement

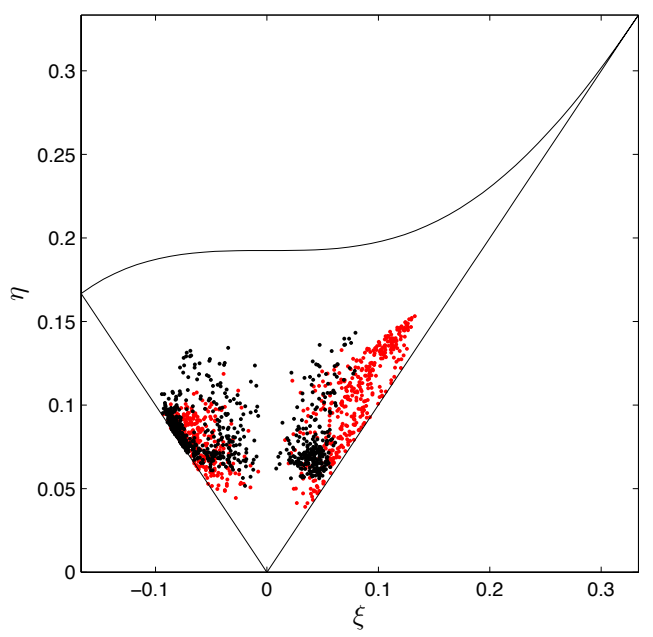

(D) Exit row of the checkerboard arrangement

Figure 4.10: Lumley triangles for each of the measurement cases. Points in black and red represent measurement windows directly upstream and downstream, respectively. Every fifth point is plotted for clarity. 
are less isotropic than the other cases. This is imparted to the flow by the alternation of rotational direction of the rotors in both the streamwise and spanwise directions, effectively stopping the formation of large-scale streamwise structures predicted in the column-by-column case.

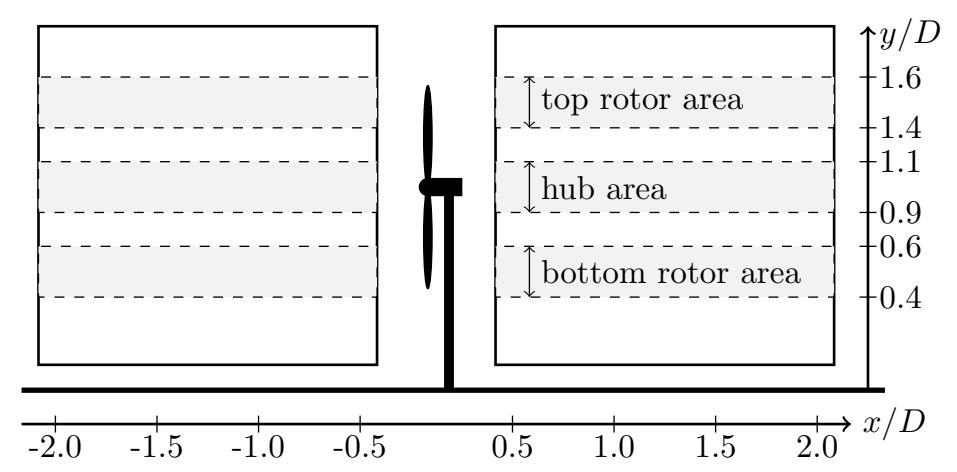

(A) Averaging areas in the wakes of exit row wind turbines.

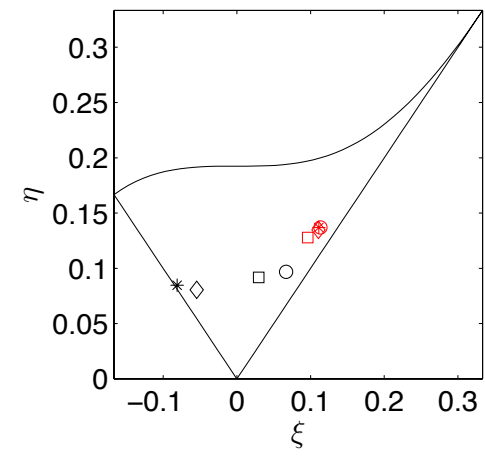

(B) AIM of the top rotor area.

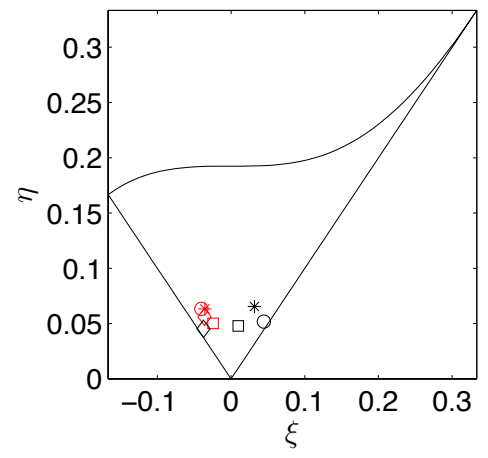

(C) AIM of the hub area.

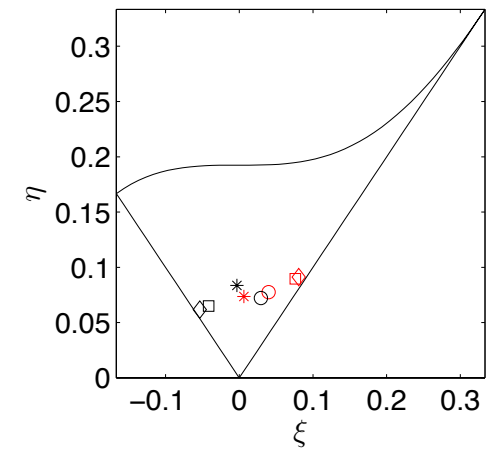

(D) AIM of the bottom rotor area.

Figure 4.11: AIMs for average invariants in areas of interest of exit row turbine wakes. Averaging areas are shown in Subfigure 4.11(A). Average invariants for all cases (o- uniform, $\square$ - row-by-row, $\diamond$ - column-by-column, $*$ - checkerboard) are shown in upstream (black) and downstream measurement (red) windows.

Previous studies regarding wind energy $[7,17]$ focus on the behavior of turbulence in key areas of interest in the wake. The flow statistics shown in Figures 4.4 through 4.9 show that there are distinct regions of behavior following the rotors. Because $U$ 
is at least an order of magnitude larger than the other components of mean velocity, these regions span the wake in the streamwise direction and are often clearly separated according to the wall-normal coordinate, $y / D$. For this reason, mean values of the invariants of $b_{i j}$ were calculated for the exit row turbines in specific areas of interest, shown in Figure 4.11(A). Mean values of $\eta$ and $\xi$ are plotted in Figures 4.11(B) through 4.11(D) for measurements upstream and downstream of each exit row turbine, represented by black and red markers, respectively. Although these values are not physical (i.e. they do not correspond to an eigendecomposition of averaged turbulent stresses) they do illustrate the overall character of the anisotropy in each case and position.

The top rotor tip demonstrates the most extreme anisotropy in each case as indicated by the magnitude of $\eta$. The mean invariants in the top rotor tip area of the wakes also show the most consistent values between cases. In the upper parts of the wind turbine canopy layer $(y / D>1.25)$, the turbulence is at a high level directly following a wind turbine and dying down in the far wake. Recalling that the inflow window to the exit row wind turbines is the far wake of the third row, it follows that the markers for each case are closer to the origin of the AIM than those in the wake, although the turbulence remains anisotropic. For the inflow, the average values of $\xi$ are negative for the column-by-column and checkerboard cases and positive for the uniform and row-by-row arrangements. The averaged values of invariants following the top rotor tips indicate that the far wakes of third row turbines show predominantly oblate spheroids for CCW rotating blades (column-by-column and checkerboard cases) and prolate spheroids for CW rotating blades (uniform and row-by-row cases).

The AIMs in Figure 4.11 serve to demonstrate an overall trend of increasing anisotropy from the hub outward to the rotor tips. This agrees well with expected re- 
sults as the hub is essentially cylindrical with a backward facing step. From this shape one expects turbulent structures to roll off from much of the perimeter. The presence of the mast contributes to the anisotropy shown in Figure 4.11(C). For all cases, $\xi<0$ following the nacelles of the turbines indicating that the turbulence there favors oblate spheroids. Upstream of the turbine, only the column-by-column arrangement of turbines exhibits compressed turbulent shapes. Each of the other cases demonstrate characteristic spheroids with stretched or prolate shapes.

Anisotropy near the bottom rotor tip in Figure 4.11(D) is in the zone of interaction between the turbine canopy layer and the boundary layer developing between successive turbine rows. The average invariants in this region of the flow show little conformity between cases in average values of $\xi$. The uniform and checkerboard cases show average invariants that are nearly the same crossing from inflow to outflow. From the contours of $\xi$ in Figure 4.9 it is evident that the behavior is quite different in the two locations and that the similarity in upstream and downstream windows is due to occurrences of both positive and negative values in the averaging areas resulting in average values of $\xi \approx 0$.

In Section 2.2 and touched upon in the discussion above, $b_{i j}$ can be described by a characteristic three-dimensional body. The eigenvalues from Equation (2.10) can be used to compose a spheroid characterizing the state of turbulence, where the radii of the spheroid are the eigenvalues themselves. This method has been used in other research $[31,35]$ to identify the anisotropy tensor using a single body rather than a series of plots or values.

In the characteristic shapes of the anisotropy tensor, a sphere describes perfectly isotropic turbulence and is invariant to rotation in any sense. Because the third eigen- 
value resulting from Equation (2.10) is always equal to the opposite of the sum of the first two eigenvalues $\left(\lambda_{3}=-\left(\lambda_{1}+\lambda_{2}\right)\right)$, the only eigenvalues forming a sphere are identically zero, as all eigenvalues are equal for isotropic turbulence. Following Equation (2.11) and discussed above, the perfectly isotropic condition would also require that the invariants $\eta$ and $\xi$ are identically zero.

The AIMs in Figure 4.10 show that the invariants for each case span much of the interior of the Lumley triangle. These data indicate that many characteristic shapes are anticipated in the wind turbine canopy layer. The averaged values of $\eta$ and $\xi$ in Figure 4.11 suggest that there are areas in the wake typified by distinct values of the two invariants. Figure 4.12 shows characteristic shapes of the normalized Reynolds stress anisotropy tensor in specific locations of the wake.

Figure 4.12(A) locates the points of interest in the flow with respect to the exit row wind turbine of the uniform case. Points are directly behind the top and bottom rotor tips and the hub of the rotor and located 1.25 rotor diameters downstream, corresponding to the center of the measurement window. The AIM in Figure 4.12(B) indicates that the invariants in the selected points match the average values for each designated area discussed in relation to Figure 4.11. According to the value of $\xi$ for each point, the characteristic spheroids should be distinctly prolate (following the top tip) or oblate (following the hub and the bottom rotor tip). The spheroids in Figures 4.12(C) through 4.12(E) demonstrate the predicted shapes. The spheroid following the hub of the rotor (Figure 4.12(D)) shows the smallest radii confirming that is the more isotropic than at the other locations. Conversely, the radii of the spheroid following the top rotor tip are the largest of those shown, indicating that the turbulence there is the least isotropic. 


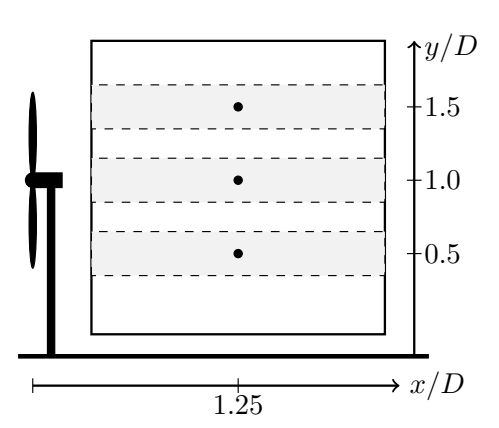

(A) Locations of characteristic spheroids wind wake areas.

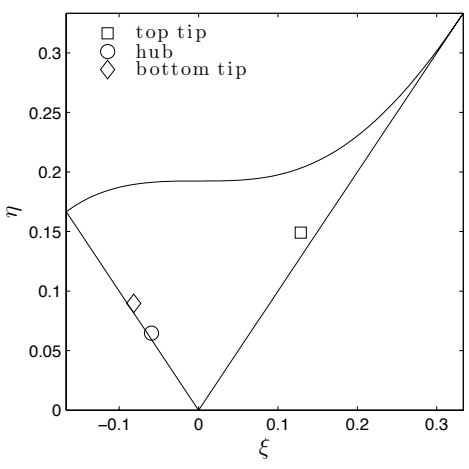

(B) AIM with invariants corresponding to points in the wind turbine wake.

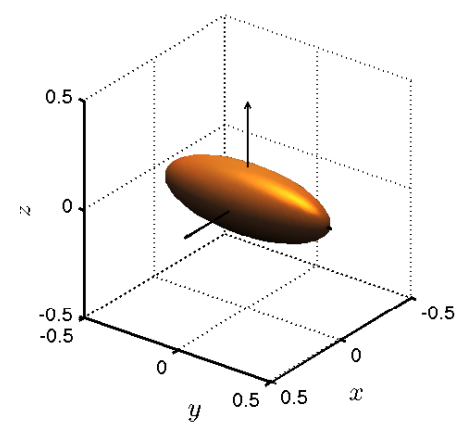

(C) Prolate spheroid typical of the top rotor area.

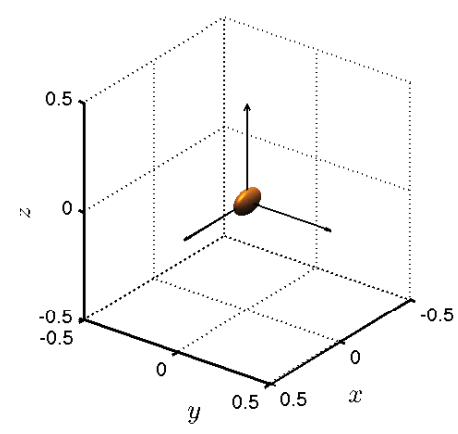

(D) Typical spheroid for the hub area.

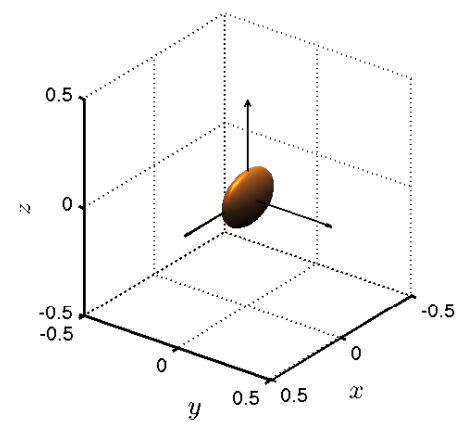

(E) Oblate spheroid typical of the bottom rotor area.

Figure 4.12: Characteristic spheroids in selected locations of the wake of the exit row turbine of the uniform case.

To further illustrate the variation of the characteristic shapes of $b_{i j}$ in the wakes, it is of interest to identify the most and least isotropic turbulence. The second invariant $\eta$ is an effective measurement of the degree of isotropy of the turbulence. Using only $\eta$ to determine to what degree of isotropy has other implications as well. The maximum value of $\eta$ allowed by the theory is $1 / 3$. At this level, the point would locate the top right of the Lumley triangle, corresponding to one-dimensional turbulence from the discussion in Table 2.1. 
Figure 4.13 shows characteristic spheroids for measurements in the wake of the exit row turbine in the row-by-row case, as it demonstrates a slightly larger span of $\eta$. The spheroids shown are selected as examples of the range of magnitudes of $\eta$ seen in the wakes. In each wake, the maximum value of $\eta$ corresponds to the least spherical (and 'least' isotropic) states of turbulence within the measurement window. Likewise, the minimum value of $\eta$ for any given measurement location corresponds to the 'most' isotropic state of turbulence. The extreme degrees of anisotropy shown in Figure 4.13 do not vary significantly between the four cases. In all cases (including those not shown) the maximum values of $\eta$ are on the order of 0.18 and the minimum values are approximately one order of magnitude smaller, i.e. $\eta_{\min } \approx 0.02$.
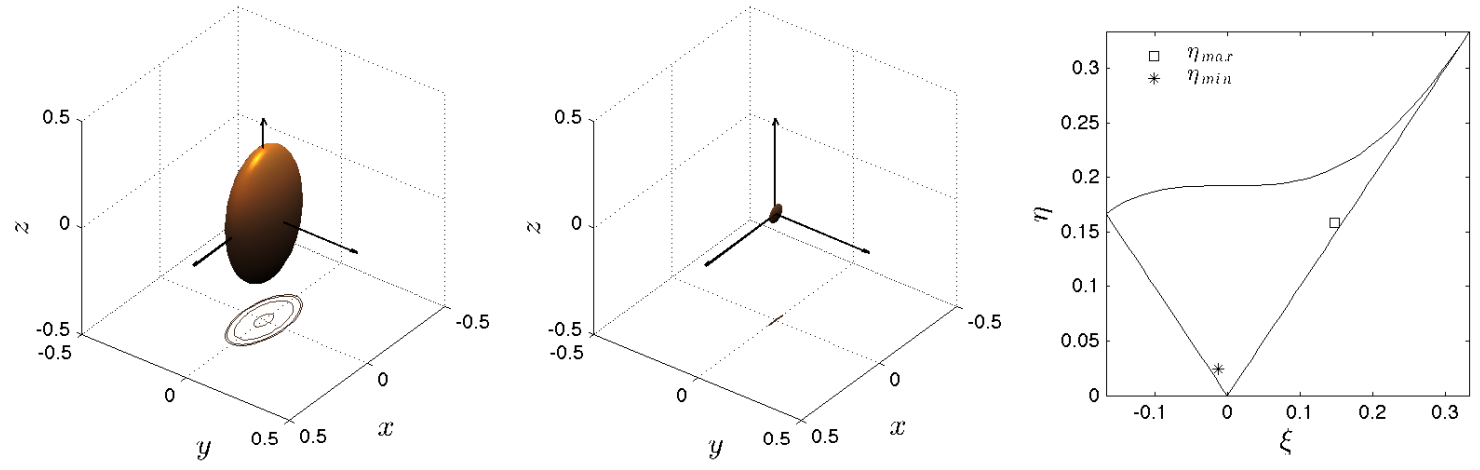

Figure 4.13: Extreme degrees of anisotropy in the wake of exit row wind turbine of the row-by-row case defined by extreme values of $\eta$. At left is the least isotropic spheroid, in the center is the most isotropic spheroid. The AIM in shows the invariants for each point as a star $(*)$ for the most isotropic state and a square ( $\square$ ) for the least isotropic state.

That perfectly one-dimensional turbulence is more anisotropic than two- or threedimensional agrees well with theory as it would be the least invariant with rotation, a critical aspect to isotropic turbulence. Stated otherwise, the vector space required to describe one-dimensional turbulence would reduce to the span of $\mathscr{R}$ in its principle 
form (from $\mathscr{R}^{3}$ for real turbulence), indicating that the rotation of the corresponding coordinate system becomes meaningless in this limit.

\subsection{Rotation of principle coordinate system}

Considering the vector basis required to describe the turbulence leads to a discussion of the eigenvectors in Equation (2.8). The principle axes output from the eigendecomposition correspond to a direct cosine matrix, denoted below as $R$. From Equation (4.2) it follows that there exists a set of rotations about each of the initial axes that map the default Cartesian axes onto a particular set of principle axes. The direct cosine matrix (or principle axes) can be expressed in terms of the Euler angles [11] as,

$$
R=\left[\begin{array}{ccc}
\cos \theta_{y} \cos \theta_{z} & \cos \theta_{y} \sin \theta_{z} & \sin \theta_{y} \\
\cos \theta_{x} \sin \theta_{z}+\cos \theta_{z} \sin \theta_{x} \sin \theta_{y} & \cos \theta_{x} \cos \theta_{z}-\sin \theta_{x} \sin \theta_{y} \sin \theta_{z} & -\cos \theta_{y} \sin \theta_{x} \\
\sin \theta_{x} \sin \theta_{z}-\cos \theta_{x} \cos \theta_{z} \sin \theta_{y} & \cos \theta_{z} \sin \theta_{x}+\cos \theta_{x} \sin \theta_{y} \sin \theta_{z} & \cos \theta_{x} \cos \theta_{y}
\end{array}\right]
$$

Extracting individual Euler angles from the direct cosine matrix is accomplished as

$$
\begin{aligned}
& \theta_{y}=\sin ^{-1}\left(R_{1,3}\right) \\
& \theta_{x}=\cos ^{-1}\left(\frac{R_{3,3}}{\cos \theta_{y}}\right) \\
& \theta_{z}=\cos ^{-1}\left(\frac{R_{1,1}}{\cos \theta_{y}}\right) .
\end{aligned}
$$

Figure 4.14 shows the unfiltered rotations output by Equation (4.3) required to map the default coordinate system onto the principle axes corresponding to the anisotropy of turbulence at any given location. 
The eigenvector output yields an orthogonal basis to describe the vector space of the stress tensor. However, it is not evident which vectors forming the principle axes correspond to each unit vector defining the original coordinate system. Whichever the order of output vectors, the coordinate space described is the same. For any three orthogonal axes, one can draw eight equally efficient sets of unit vectors if one ignores the algebraic signs of the axes. Each of these can be further permuted the names of each vector could be switched) to yield 24 sets of unit vectors describing the same right-handed coordinate system. For this reason, in the following it is assumed that the default output of the decomposition yields vectors in the order of $x^{\prime}, y^{\prime}$, and $z^{\prime}$, as in Equation (2.8).

To determine where the principle axes reside with respect to the original coordinate system, a single vector is defined central to the coordinate system. This characteristic vector, $\vec{r}$, is defined such that its magnitude is unity and it is located equidistant from each of the unit vectors defining the coordinate axes as,

$$
\vec{r}=\frac{1}{\sqrt{3}}\left\langle\vec{e}_{x}, \vec{e}_{y}, \vec{e}_{z}\right\rangle,
$$

where $\vec{e}_{i}$ are unit vectors in the $i$ direction.

The vector, $\vec{r}$, can be subjected to the three rotations shown in Figure 4.14. When $\vec{r}$ resides in a given octant, it can be said that the principle coordinate system resides mainly in the same octant. The three dimensional Cartesian system is shown with conventionally defined octants in Figure 4.15(A). Rotating a set of unit vectors according to the Euler angles shown in Figure 4.14 leads to the transformed system shown in Figure 4.15(B). The original coordinate system is designated with black arrows and the principle axes are shown in gray and labeled with prime notation. The characteristic vector 

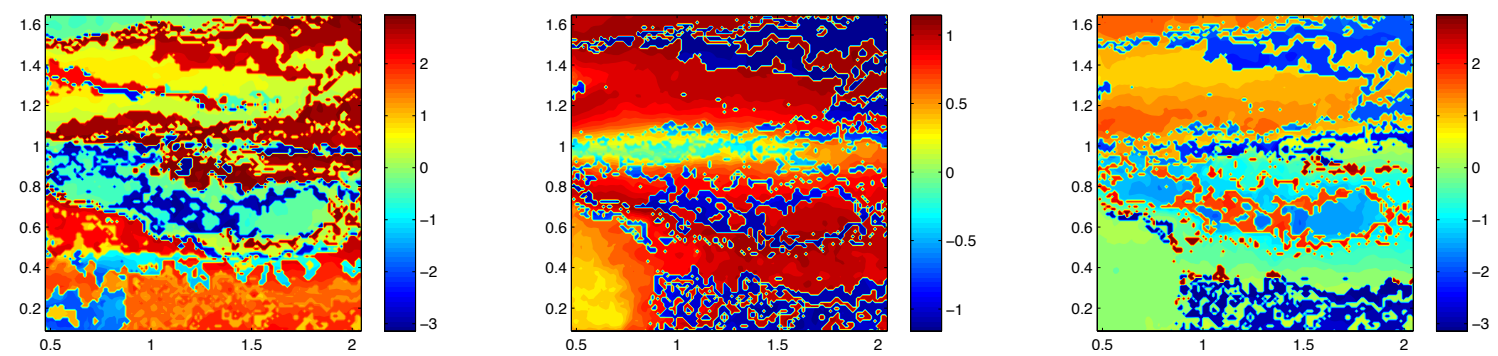

Figure 4.14: Unfiltered rotational angles of principle axes about Cartesian axes. Data pertains to the exit row of the uniform case. From left to right are rotations about the $x, y$, and $z$ axes, $\theta_{x}, \theta_{y}$, and $\theta_{z}$ respectively.

$\vec{r}$ is subjected to the same rotations and is likewise designated with a prime. The main advantage to using $\vec{r}$ to identify the residence octants is that it is independent of the potential permutation of the axes discussed above.

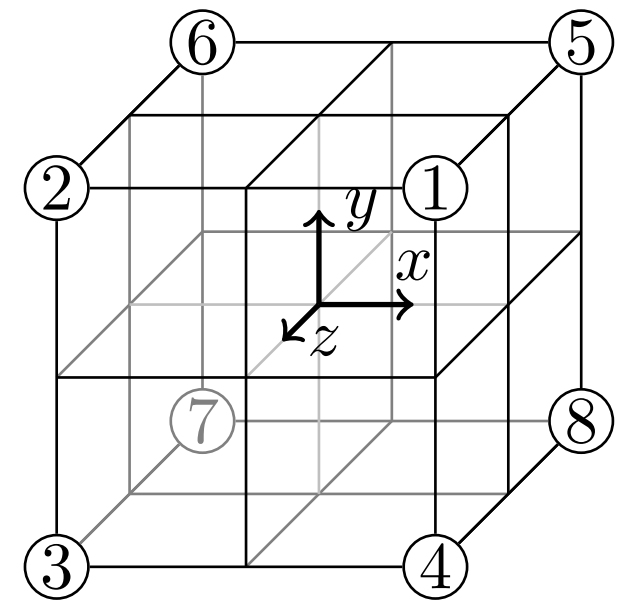

(A) Octants defined in Cartesian coordinate system.

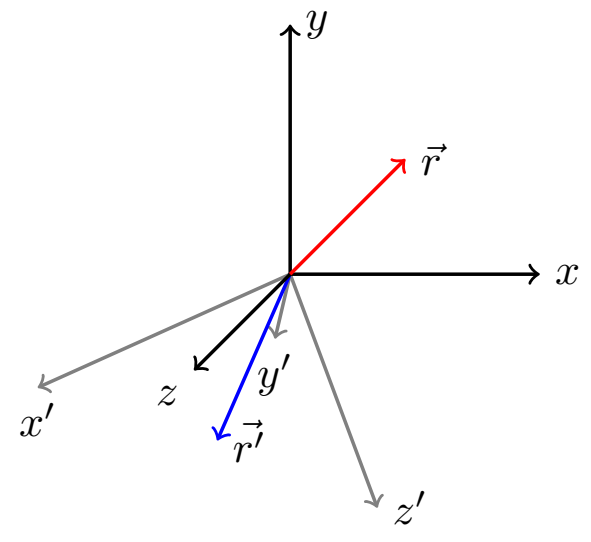

(B) Characteristic vector, $\vec{r}$, for principle coordinate system.

Figure 4.15: Octants of the Cartesian coordinate system defined by the sign of standard vectorial components and rotated coordinate system and characteristic vectors of both the Cartesian system and the principle axes.

To achieve a clearer picture of where the principle axes reside for each measurement area, the occupation of the principle axes in each of the octants is tallied within 
the domain, shown in Figure 4.16. Similar to the analysis of the invariants of $b_{i j}$, the residence of the characteristic vector (and principle coordinate system) is tallied in each of the averaging areas denoted in Figure 4.11(A). Relative occupations of the transformed coordinates in each of these areas are shown in Figures 4.16(B) through 4.16(D).

Figure 4.16(A) shows that in the entire measurement domain, the principle coordinate system of the turbulence in all cases are dominantly found in octants 1, 2, 5, and 6 indicating that $\theta_{x}<\pi / 4$. The total rotation of $\vec{r}$ is nearly always ( $\left.\sim 95 \%\right)$ constrained to the interval $-\pi / 2<\theta<\pi / 2$. Over the entire measurement domain, turbulence in the wakes of all cases reside in the fifth octant to a large degree. Turbines with CW spinning blades (the uniform and checkerboard cases) show a greater tendency to occupy the second octant and turbines with CCW rotors occupy the first octant more. For all cases, the residence in the third octant is identically zero. The residence in the seventh octant is low enough $(\sim 0.1 \%)$ to suggest that any rotation there is the result of numerical error.

In the region of the wake downstream of the top rotor tip, the principle axes of turbulence following CW blades occupy the second octant in approximately $35 \%$ and $65 \%$ of measurement locations for the uniform and checkerboard cases, respectively. Following the top rotor tip in the CCW cases, between $20 \%$ and $30 \%$ of principle systems remain in the first octant whereas $60 \%$ to $70 \%$ of coordinate systems reside in the fifth octant.

In the hub region of the wake (Figure 4.16(C)), CW rotating turbines favor the second, fifth, and sixth octants fairly evenly. Turbines with CCW rotational sense favor the first and second octants. Recalling the AIMs from Figure 4.11, the averaged invariants 


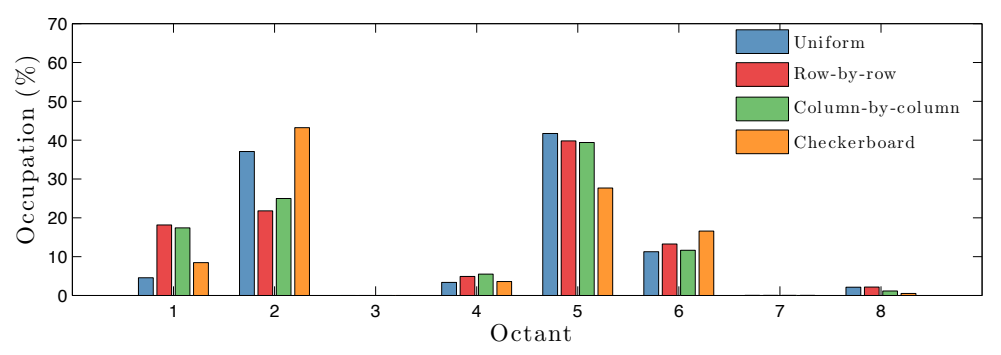

(A) Aggregate occupation.

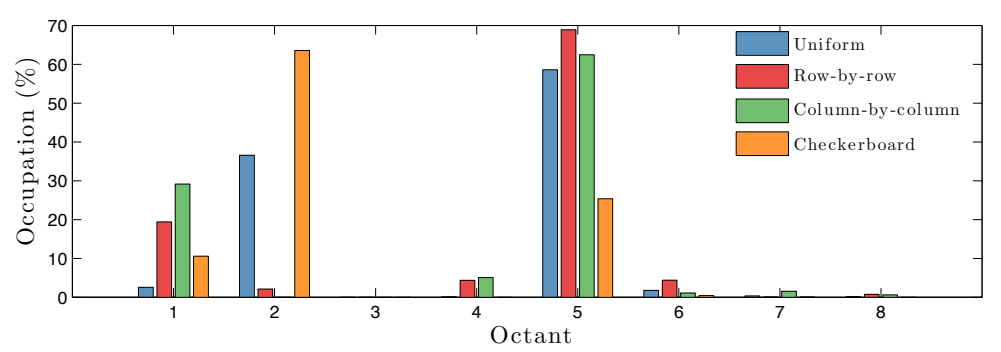

(B) Top rotor area.

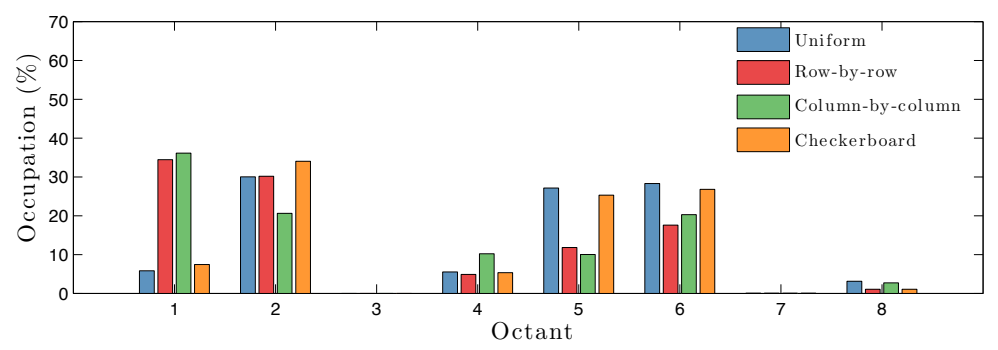

(C) Hub area.

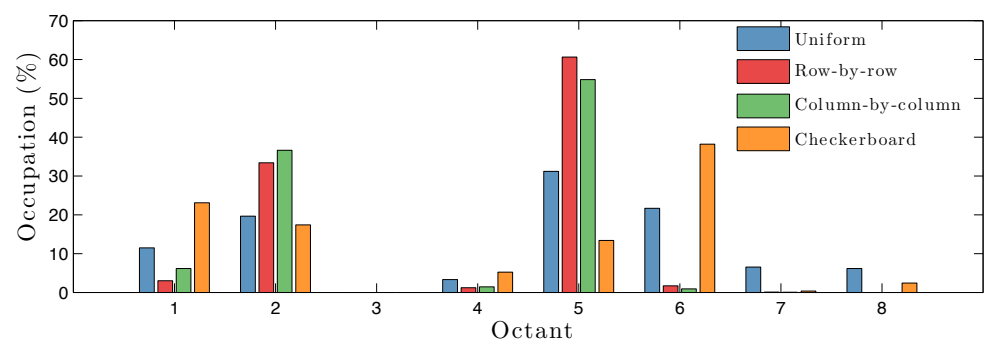

(D) Bottom rotor area.

Figure 4.16: Percent occupation of principle axes in Cartesian octants. Subfigures 4.16(B) through 4.16(D) show the occupation of principle axes in areas of interest in the wake and Subfigure 4.16(A) shows the occupation for the entire measurement area. 
in the hub area of the wakes are close to the origin, relative to other parts of the wake. These observations together imply that principle axes corresponding to isotropic turbulence demonstrate less localization to any particular octants than those associated with highly anisotropic turbulence. Following the hub, the principle coordinate systems corresponding to the uniform and checkerboard cases occupy the second, fifth, and sixth octants approximately for $30 \%$ of measurement points. The row-by-row and column-by-column cases are found more in the first $(\approx 35 \%)$, second $(\approx 30 \%)$, and sixth $(\approx 20 \%)$ octants. The change from the first to fifth octants indicates the change of rotation of the rotors from CCW to CW respectively.

The data for the Euler angles show discontinuities throughout the measurement fields resulting from the numerical eigendecomposition and conversion. The decomposition outlined in Equation (2.8) seeks the most efficient vector basis for the turbulent stress tensor rather than the continuous changes from point to point, and thus is subject to some error when converted into Euler angles. The default output interval of the inverse sine and cosine functions from Equation (4.3) is from $-\pi$ to $\pi$, leading to discontinuities in the rotations of the principle axes shown in Figure 4.14 when $\theta_{i} \approx \pm \pi$.

In order to clarify the the rotations of the principle axes, a treatment was applied to the Euler angles. The residence of the characteristic vector described in Figure 4.16 indicates that the principle axes favor octants 1,2, 5, and 6. Treatment of the axes was conditioned based on this observation. When $\vec{r}$ resided in octants $3,4,7$, or 8 , the corresponding principle axes were reflected about the origin, effectively limiting $y^{\prime} \geq 0$. The residence of the treated characteristic vector are shown in Figure 4.17. The imposed treatment leads to the lesser occupied octants being added to their respective 
reflections across the origin. Referring to Figure 4.15(A), this leads to the residence of octant 7 to be added to that of octant 1 , for example.

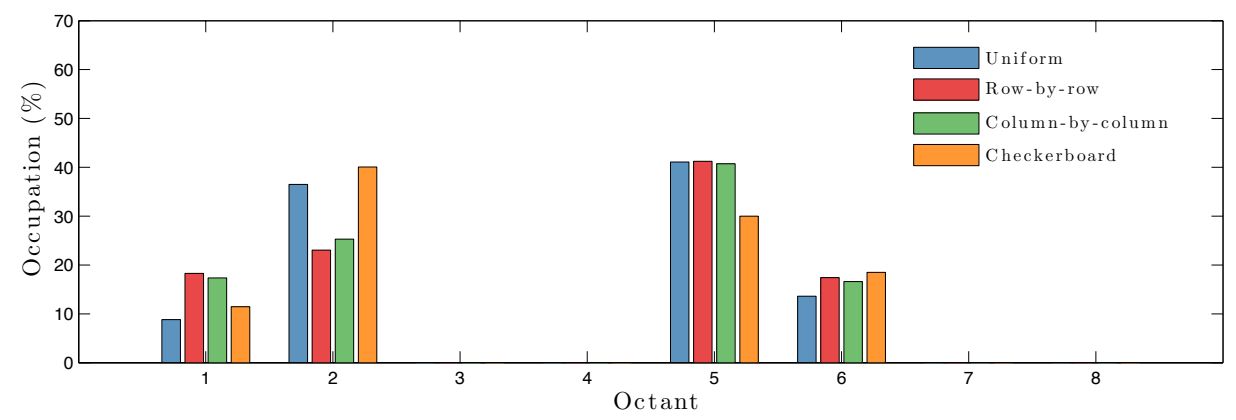

Figure 4.17: Residence of characteristic vector $\vec{r}$ in Cartesian octants after application of treatment to Euler angles.

Results of these filters are shown in Figure 4.18. In the figure, the absolute values of $\theta_{x}$ and $\theta_{z}$ are shown. The second treatment is done only for the purposes of visualization and makes the contours more continuous while maintaining discernible differences case to case.

It should be noted here that these are strong treatments and that in conditioning these fields there is a risk of losing information. These treatments constrain the rotations of the coordinate system to the octants common to all cases (octants 1, 2, 5, and 6), defined in Figure 4.15(A) as $y \geq 0$. Although the treatment of Euler angles is strong, the imposed constraints provide a clearer picture of the coordinate rotations. Differences between cases and rotational senses of the rotors remain evident after treatment.

Seen in the left column of Figure $4.18, \theta_{x}$ shows minimum magnitudes of rotation immediately following the mast of each wind turbine. There is also a range of small rotation $\left(\theta_{x}<\pi / 4\right)$ following the nacelles. In the cases of CW rotating blades (uniform and checkerboard cases, top and bottom rows, respectively) this area shows greater 

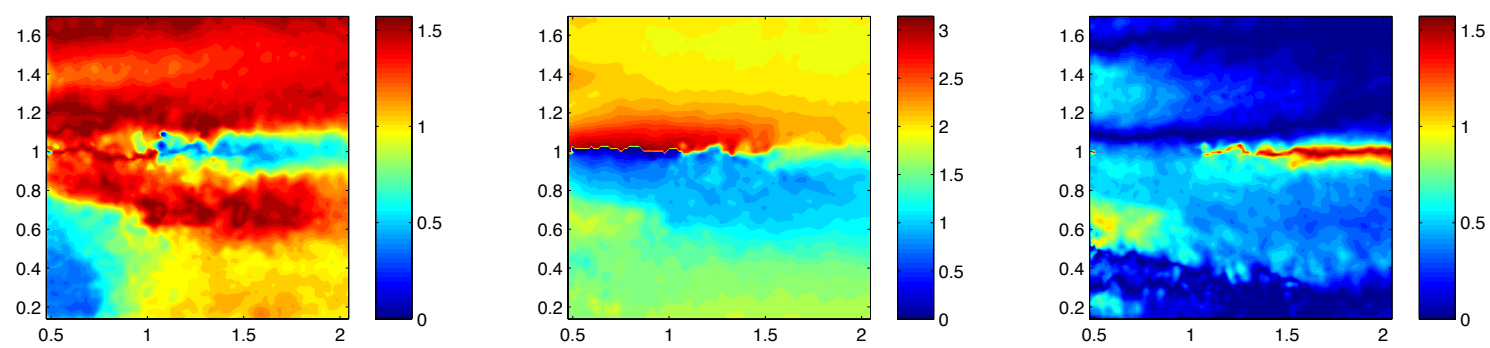

(A) Uniform case.
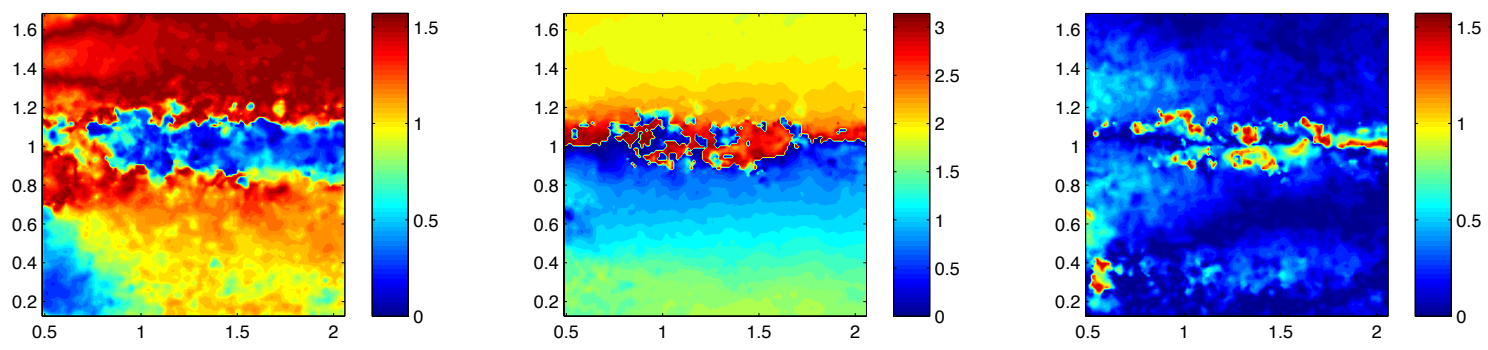

(B) Row-by-row case.
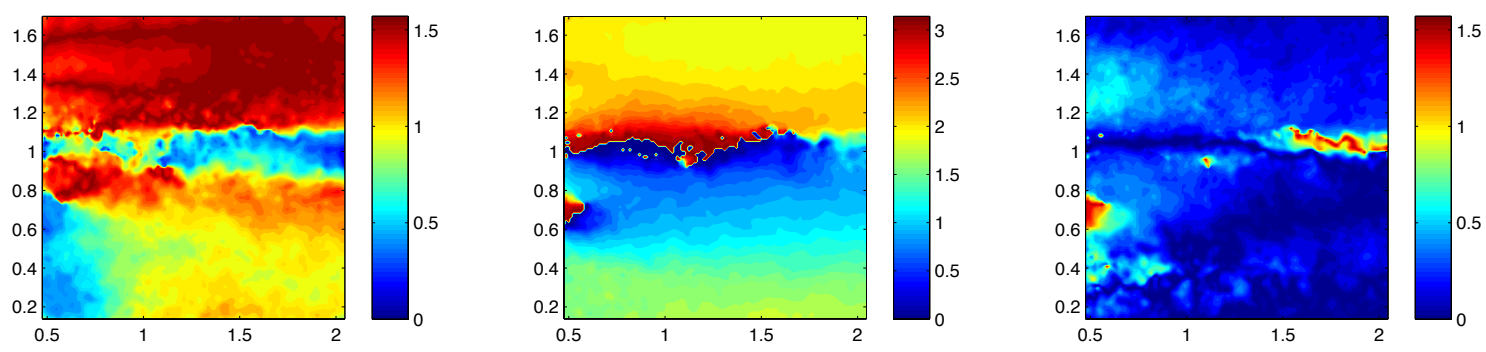

(C) Column-by-column case.
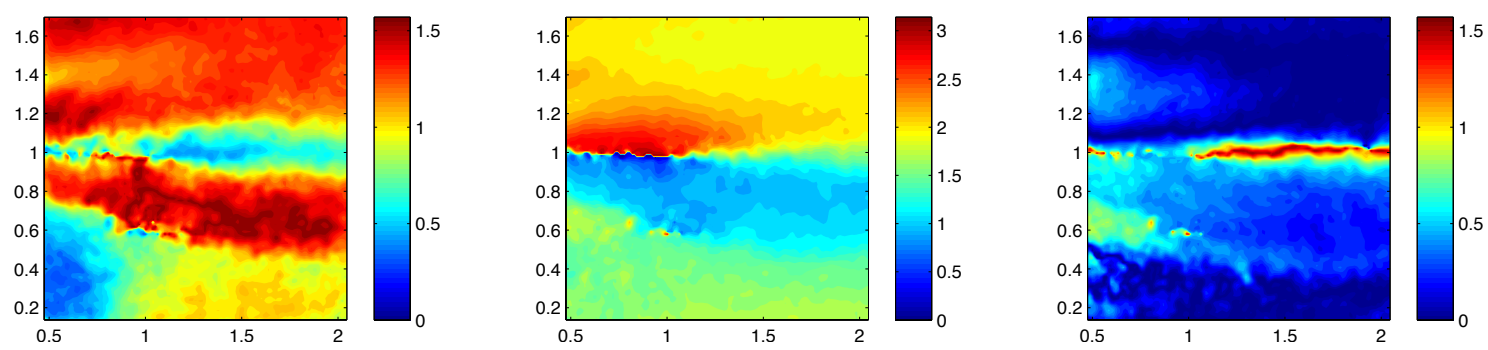

(D) Checkerboard case.

Figure 4.18: Euler angles (about default axes) of rotation of principle coordinate system. Columns from left are $\theta_{x}, \theta_{y}$, and $\theta_{z}$ (rotations about the streamwise, wallnormal, and spanwise coordinates, respectively). 
rotation than for the CCW cases. The octant occupation delineated in Figure 4.16 indicate that for all cases the characteristic vector shows less localization to any particular orientation in this region of the flow. Following the bottom rotor tip of the CW cases, there is a region of fairly large rotations about the $x$ axis not seen for CCW blades. Looking to the wake following the top rotor tip, $\theta_{x}$ is similar for each case but remains at the upper limit of $\pi / 2$ in a larger part of the wake for the CCW blades.

In Figure 4.18 it can be seen that $\theta_{z}$ is very similar for all cases, with a region of maximum rotation trailing the hub for $x / D \geq 1$. The regions of large rotation about the $z$ axis of the Cartesian system are very localized to the center of the measurement domains. The treatment from above shows that for much of the measurement domain, $\theta_{z}$ is small. There are noticeable differences between the cases based on direction of rotation, however. CW blades show an increase rotation about $z$ following the bottom rotor blade over that following the top rotor tip. Although small in magnitude, the CCW blades show nearly even rotations about $z$ following both rotor blades.

The most extreme differences between cases are seen in $\theta_{y}$. In all cases there are distinct areas following the hub where rotations are maximum directly above the hub and minimum directly below. Moving both upward and downward away form the hub, every case shows that rotation of the principle axes tends toward a moderate rotation. The region of small rotations below hub height in the wake is accentuated in the CCW blades (row-by-row and column-by-column cases, middle two rows). For the uniform and checkerboard cases, the region of small $\theta_{y}$ below the hub matches the region of large $\theta_{x}$ very closely.

The eigenvalues defining the radii of the characteristic spheroids above and principle axes defining their orientation result from the same decomposition. Each spheroid 

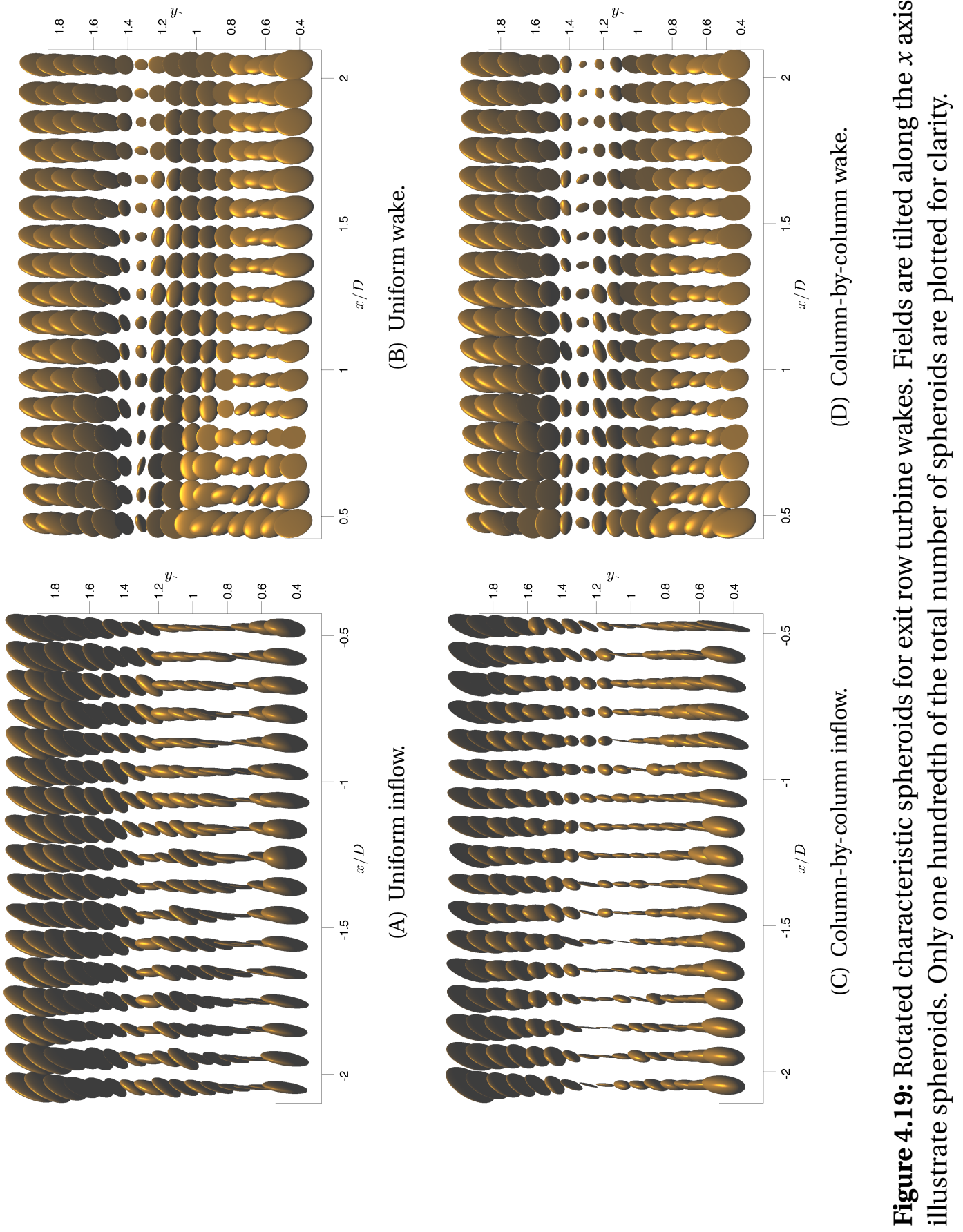
can be rotated about its center according to the Euler angles shown in Figure 4.18. The synthesis between the degree of anisotropy and alignment of the principle axes can be seen in Figure 4.19. The radii of each spheroid are taken directly from the eigenvalues from Equation (2.10). The rotations of each spheroid are taken from the filtered angles, $\theta_{x}, \theta_{y}$, and $\theta_{z}$.

As discussed pertaining to the spheroids shown in Figure 4.13, a truly isotropic state of turbulence is described by $\lambda_{i}=0$. Based on this, the smaller characteristic shapes shown in Figure 4.19 can be said to be 'more' isotropic than ones with larger radii. The figure shows rotated characteristic spheroids in both inflow and wake locations for the uniform (Figures 4.19(A) and 4.19(B)) and column-by-column (Figures 4.19(C) and 4.19(D)) cases. These configurations of the wind turbine array were chosen based on the differences seen in the contours of the invariants $\eta$ and $\xi$ as well as the rotational fields from Figure 4.18. In Figure 4.19, spheroids are composed for every eighth measurement location in the streamwise and spanwise location for clarity.

The inflow windows of the two cases demonstrate highly organized turbulent flows. Figures $4.19(\mathrm{~A})$ and $4.19(\mathrm{C})$ both demonstrate large prolate shapes near the wall, agreeing well with the predictions of the invariants. In both cases, the characteristic spheroids get smaller approaching $y / D=1$. The inflow of the column-by-column arrangement shows smaller spheroids and a greater tendency toward oblate shapes. The spheroids near the top of the rotor are much larger than near the hub area. The orientation of spheroids is consistent across the fields. The orientations of the spheroids show little variation in the streamwise direction and larger changes progressing in the wall-normal direction.

The wake areas in Figures 4.19(B) and 4.19(D) show much more variation in the 
characteristic shapes and orientation of the anisotropy field. The most isotropic states of turbulence follow the hubs of the turbines, in agreement with the averaged invariants shown in Figure 4.11 and the contours of $\eta$ from Figure 4.8. Both configurations of the turbine array demonstrate similar behavior in Figure 4.19 wherein there is a stretching of the spheroids (tending toward prolate shapes) trailing the top rotor tip, and a flattening of spheroids (tending toward oblate shapes) following the bottom rotor tip except very near to the wind tunnel floor.

The rotations of spheroids in the figure appear quite similar as well, due to the treatment of the Euler angles above. Composing the tensor field in Figure 4.19 with the unfiltered Euler angles leads to obscured trends as the rotations show sharp discontinuities in the measurement field. Even with the treatment the rotations of the spheroids show dependence on the rotational sense of the rotor blades. The uniform case with a CW rotor, Figure 4.19(B), shows less rotation about the $x$ axis than does the CCW rotor of the column-by-column case in Figure 4.19(D) following the top rotor blade. In a different manner, the rotation for the uniform case is increased following the bottom rotor blade. The rotation about the $y$ axis is evident in the wakes by the coloring of the spheroids. Below hub height where $\theta_{y}$ is minimum just below the hub and increasing downward, the spheroids show their well lit sides and are lighter in color. The opposite is seen above the hub, where the spheroids have been rotated up to $\pi$ radians about the $y$ axis. There the 'back' sides of the spheroids are shown as darker in color. 


\subsection{Flux and production of kinetic energy}

The flux of kinetic energy, $F_{12}$, shown in Figure 4.6 quantifies how much kinetic energy from the bulk flow above the turbine canopy is transported by large-scale turbulence into the momentum-deficit area of the wake [4,15-17]. Comparing the contour plots showing $F_{12}$ and $\xi$ from Figure 4.9 indicates that there is a connection between the two quantities. In its complete formulation, the flux term is second-order tensor multiplying the mean velocity and the Reynolds stress tensor. As $\xi$ is an invariant of the Reynolds stress tensor normalized and put into its principle components, a connection between the two is intuitive, although the exact form of the connection is not apparent.

Figure 4.20 demonstrates correlations between terms from Equation (2.2) and the invariants of $b_{i j}$ and illustrates that there are differences from case to case. CCW rotational cases (the row-by-row and column-by-column cases; red and green, respectively) exhibit larger magnitudes of both $F_{12}$ and $P_{12}$ than the CW cases. However, because there are theoretical limits for the invariants $\eta$ and $\xi$, the vertical spread of points in the figures are nearly equal.

The plots shown in Figure 4.20 use only data for the upper rotor blade and above the canopy where $y / D \geq 1.25$. Generally, $\eta$ seems to be in good agreement with both $F_{12}$ and $P_{12}$ in this region of the wake. In the scatter plot of $F_{12}$ vs. $\eta$ (Figure 4.20(A)) the four array configurations are distinct from one another. Across the plot are the uniform, checkerboard, column-by-column, and row-by-row cases in ascending order of flux of kinetic energy. All four cases show the same range of $\eta$ and a linear regression of points shows a slope of 0.075 changing less than $10 \%$ between cases.

The scatter plot of $P_{12}$ versus $\eta$ (Figure 4.20(C)) shows the same order of array con- 


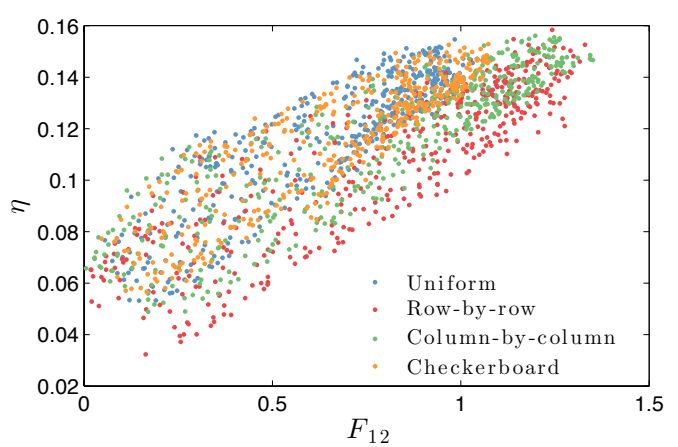

(A)

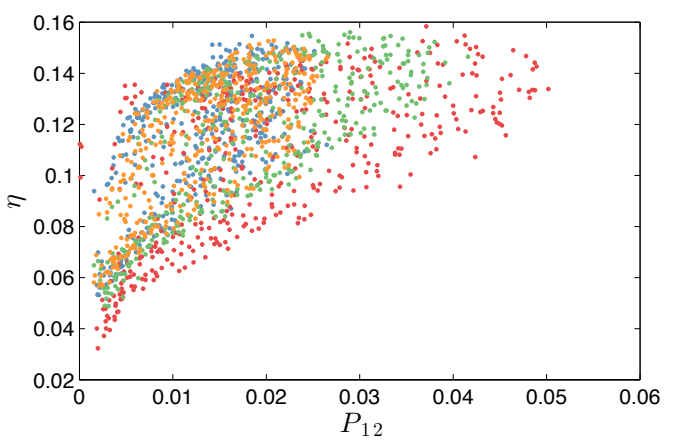

(C)

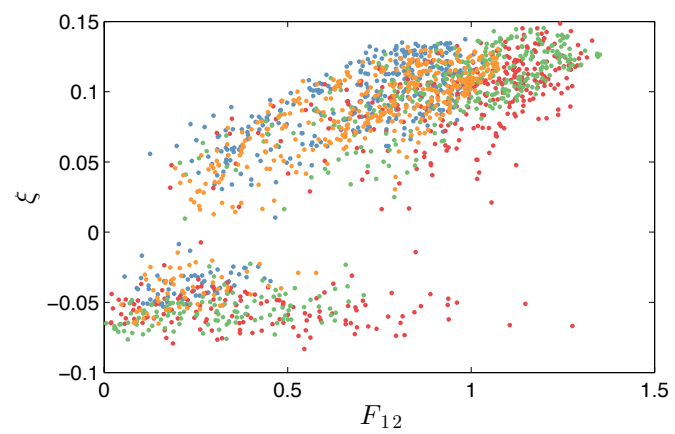

(B)

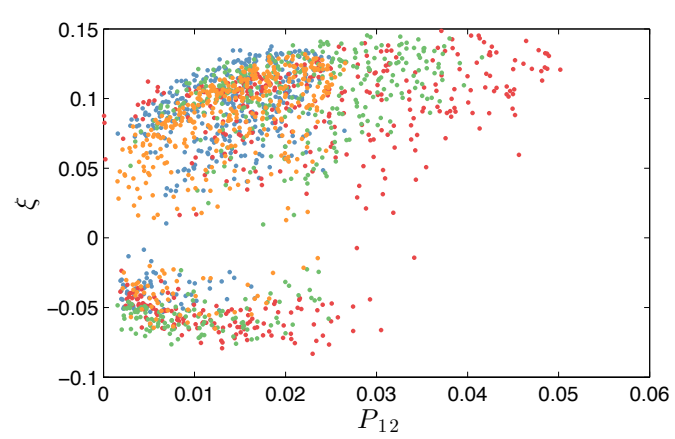

(D)

Figure 4.20: Scatter plots of the flux and production of kinetic energy against the invariants of $b_{i j}$.

figurations with greater divergence from case to case, as the production varies more between cases than does the flux. The points of the uniform and checkerboard cases are nearly identical for these quantities. The column-by-column and row-by-row cases show maximum values of $P_{12}$ that are $60 \%$ and $80 \%$ larger than the CW rotors, respectively. The best form of regression between $P_{12}$ and $\eta$, as shown in Figure 4.20(C), is much less obvious than between $F_{12}$ and $\eta$, here shown in Figure 4.20(A).

Plots containing $\xi$ show several distinct regions when plotted against either $F_{12}$ or $P_{12}$. Figures 4.20 (B) and 4.20(D) indicate that there may be two regimes of correlation, depending on the algebraic sign of $\xi$. Regarding the comparison of $F_{12}$ and $\xi$ (Figure 
4.20(B)), the correlation when $\xi>0$ is very similar from case to case, as seen with the correlation of $F_{12}$ and $\eta$. In the region of $\xi<0$, the variation of $\xi$ is very small as $F_{12}$ increases; $\xi$ is almost constant at value of -0.05 .. The row-by-row case shows an especially large downward flux of kinetic energy associated with negative values of $\xi$, as large values of $F_{12}$ are reflected in red spanning up to 1.25 , thus their magnitudes is more than twice compared with the other cases. These points exist in the non-overlap region in Figure 4.21(B).

A cursory scan of the contours for each quantity indicates that the regions in which $F_{12} \geq 0$ correspond to the regions of the wake in which $\xi \geq 0$. A demonstration of this is seen in Figure 4.21 where a threshold was set based on the algebraic signs of each quantity. The threshold maps shown in the left and center columns of the figure are the algebraic signs of $F_{12}$ and $\xi$, respectively. Viewing the two quantities in this way makes determining their overlapping regions clear. The right column of subfigures indicates the correlation of the two quantities. For the overlap, white and black areas indicate that the signs of $F_{i j}$ and $\xi$ are the same behind the exit row turbines and either positive or negative, respectively. The areas where the signs of the two quantities are not the same are shown in gray. From the figure, it can be seen that the match of signs is more prevalent in the uniform and checkerboard cases (CW rotation) than it is in the row-by-row or column-by-column cases (CCW rotation). The difference in overlap can be attributed mainly to the sign of $\xi$, as the contours denoting the algebraic sign of $F_{12}$ are very consistent between cases.

Analyzing only the signs of $F_{i j}$ and $\xi$ dismisses information regarding the magnitudes of either, yet the overlap regions indicate strong behavioral tendency in the wakes of wind turbines. A positive value of $F_{i j}$ indicates a downward flux of kinetic 

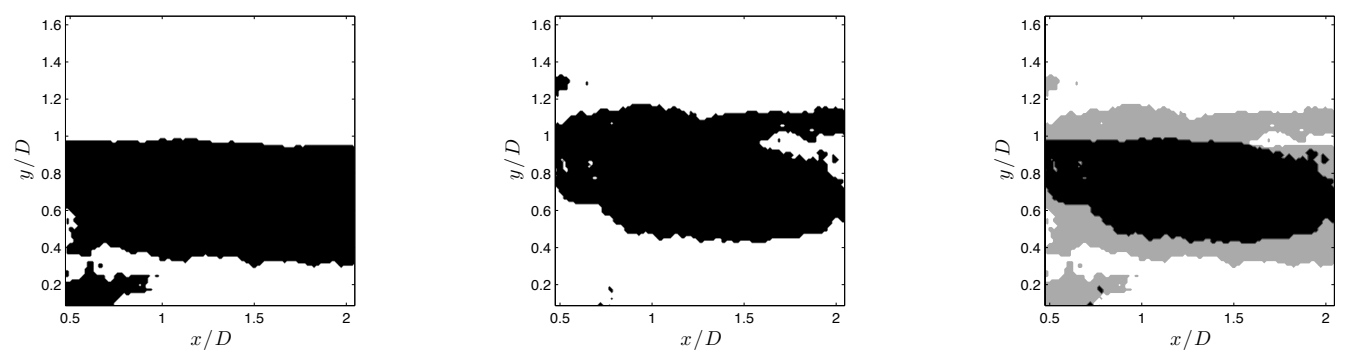

(A) Uniform case.
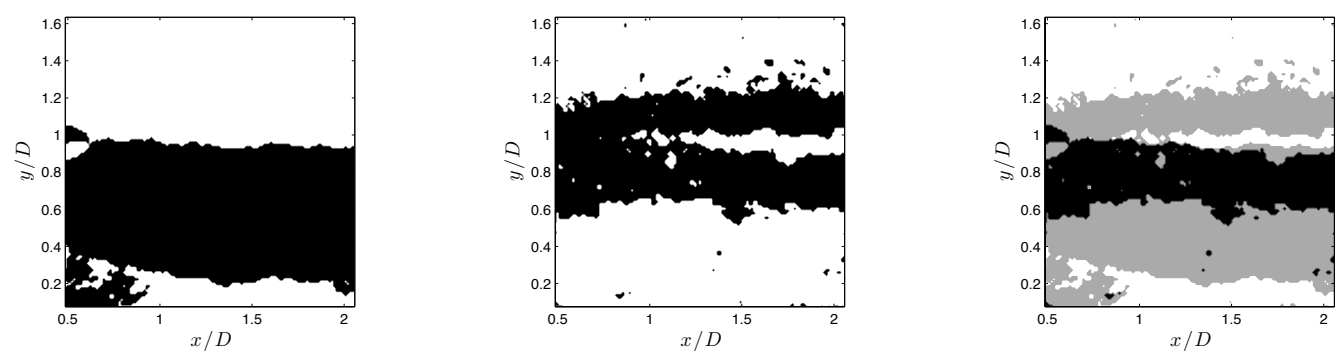

(B) Row-by-row case.
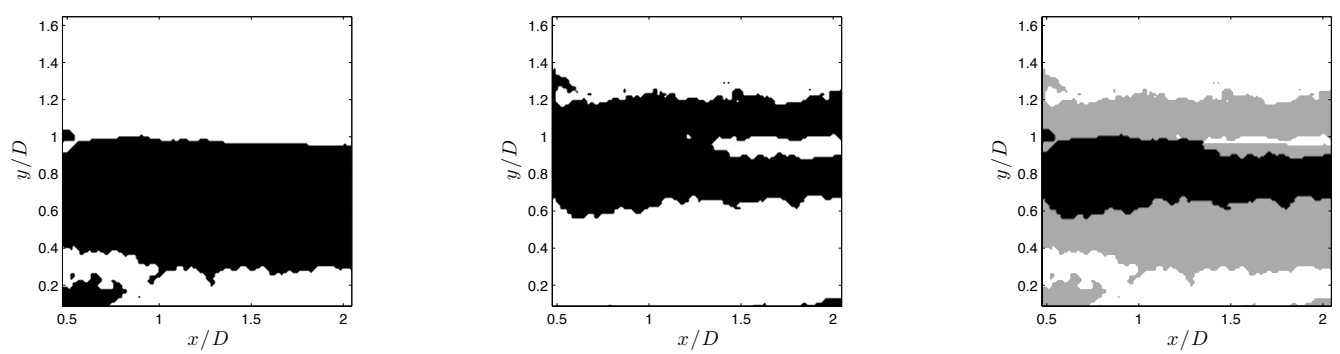

(C) Column-by-column case.
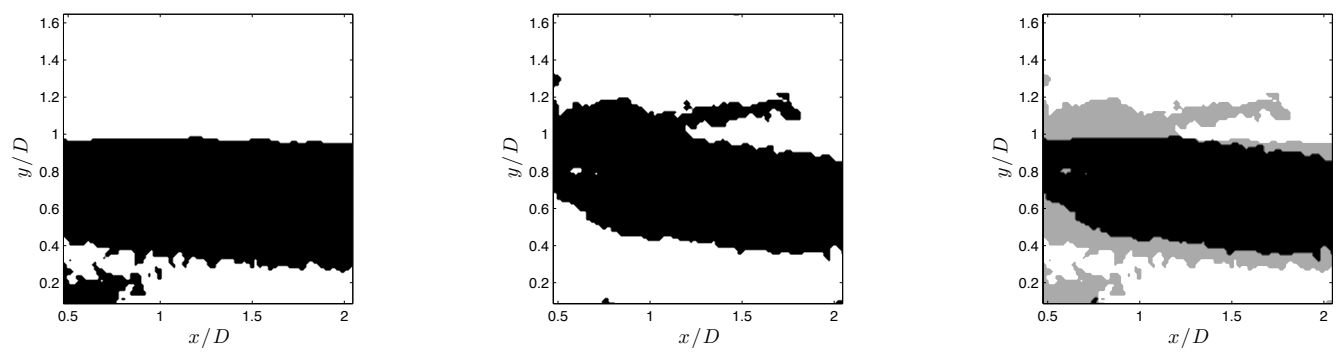

(D) Checkerboard case.

Figure 4.21: From left are the algebraic signs of $F_{12}$ and $\xi$ and their regions of overlap. In the right column, overlaps are positive or negative and matching (in white and black, respectively) or not matching (in gray). 
energy into the wake and a negative value indicates an upward flux. Coupling this with the sign of $\xi$ indicates that when the flux of kinetic energy is downward, it is overwhelmingly associated with turbulence whose characteristic spheroids tend to be prolate and exhibit a single eigenvalue larger than the other two. Likewise, upward flux is coupled with turbulent structures forming oblate spheroids and exhibiting one eigenvalue smaller than the other two. Stated otherwise, downward flux is associated with turbulence with one dominant component of the stress tensor and upward flux is associated with two dominant components.

The regions of Figure 4.21 denoted by gray contours are regions in which the direction of flux and the shapes of the characteristic spheroids have the opposite association. Gray areas above the hub $(y / D>1)$ are oblate spheroids and contributing to downward flux. Below the hub $(y / D<1)$, the gray denotes prolate spheroids associated with upward flux. There is a consistent pattern moving upward through the overlap contours (white, gray, black, gray, white). This pattern suggests that the relationship between $F_{12}$ and $\xi$ is highly dependent on $y$ and has a complex form.

A similar approach is taken for the in-plane production of turbulence kinetic energy, $P_{12}$, and the second invariant of the normalized Reynolds stress tensor, $\eta$. The magnitude of $\eta$, ranging from 0 to $1 / 3$, is an indication of the anisotropy of turbulence. As with the flux, $P_{12}$ includes the Reynolds stress tensor in its full composition and as such, connection between it and the invariants is anticipated.

In Figure 4.22 , the quantities compared $\left(P_{12}\right.$ and $\left.\eta\right)$ are by definition always positive, and the same comparison made in Figure 4.21 is an inappropriate choice. A threshold for comparison is necessary for each quantity. Noting that in the contour plot of $P_{12}$ and $\eta$ (Figures 4.7 and 4.8) there is a correlation between the regions of rel- 

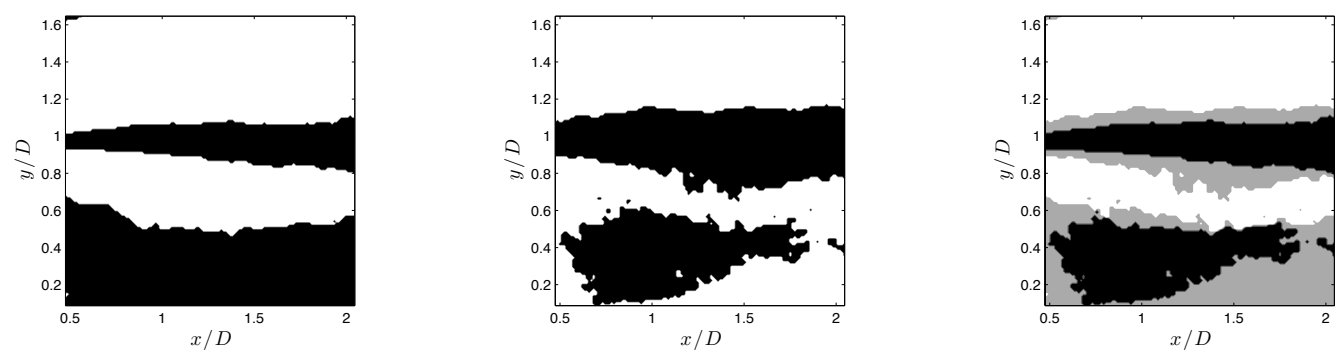

(A) Uniform case.
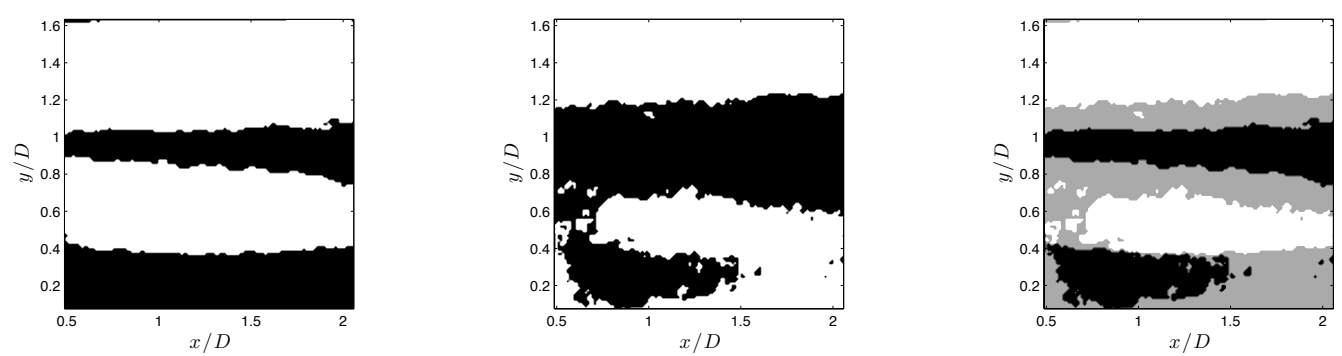

(B) Row-by-row case.
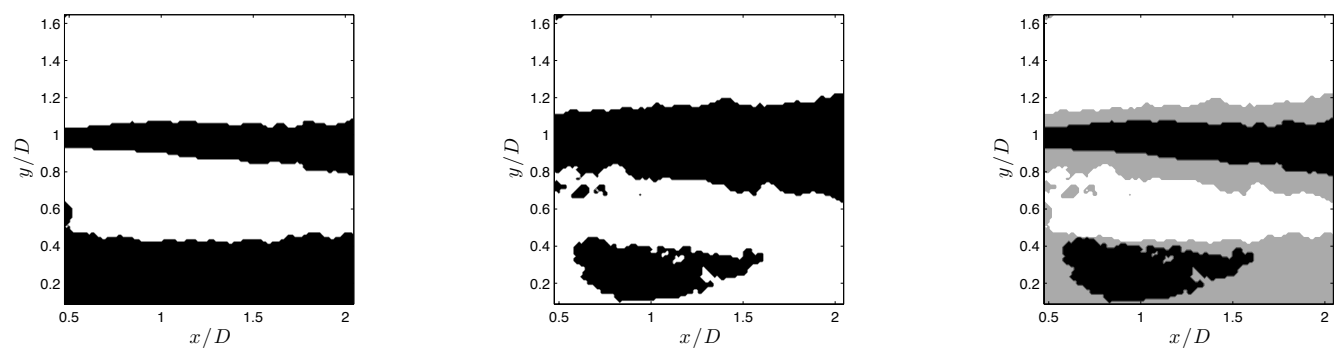

(C) Column-by-column case.
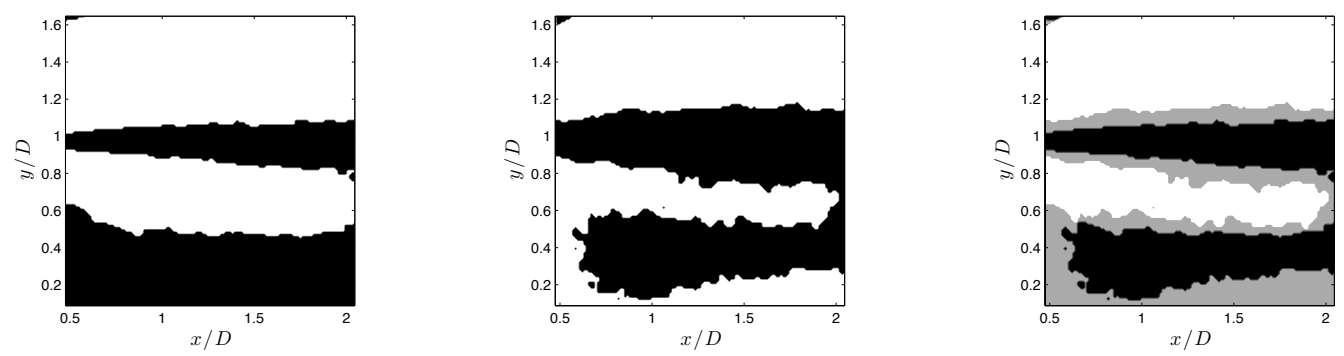

(D) Checkerboard case.

Figure 4.22: Threshold plots of $P_{12}$ and $\eta$ in the left and center columns respectively. The right column shows overlapping areas as positive or negative and matching (in white and black, respectively) or not matching (in gray). 
atively high production and larges values of $\eta$. In the left column of Figure 4.22, values of $P_{12}$ less than $1 \%$ of the maximum are shown in black and considered to be regions of low production. The threshold for $\eta$ was set to the mean value in each of the wakes.

The overlap of the threshold conditioned production and second invariant indicate that there is almost total agreement in the region of the wake following the top rotor tip. This indicates that the productive regions (in the sense of turbulence kinetic energy) correlate with regions of anisotropic turbulence. From the contour plots of $U$ (Figure 4.2) vertical gradients in the mean flow are associated with productive and anisotropic regions in the wakes, as predicted by the transport equations for Reynolds stresses. The overlap maps in the right column of Figure 4.22 also show that the central region of the wake (following the hub) are regions of low production and that the turbulence there is much more isotropic.

The significance of the gray 'mismatch' areas from Figure 4.22 is less clear than in was in the overlaps of $F_{12}$ and $\xi$. The threshold for production was set to $1 \%$ of the maximum value of $P_{12}$ allowing the designation of black contours in the left column as low production. The threshold for $\eta$ as the mean value roughly classifies the anisotropy seen in the wakes as either 'weak' or 'strong' but does not result from an obvious division of values. In the right column of subfigures, the gray contours following the hub $(0.75<y / D<1.25)$ correspond to high production but weak anisotropy. In the area of the wake below the rotor $(y / D<0.5)$, gray contours are areas of low production and strong anisotropy. The contours of $\eta$ in Figure 4.8 indicate that the transition between weak and strong is continuous. Nevertheless, the overlap of the thresholds is consistent with matching areas taking up approximately $75 \%$ of the wake areas. The overlapping thresholds of terms from the mean mechanical energy equation and the invari- 
ants of the normalized Reynolds stress anisotropy tensor (Figures 4.21 and 4.22) are sufficient to demonstrate that there are correlations between the quantities. Although the functional relationship between all quantities is not undertaken in this work.

\subsection{Power and efficiency}

Important considerations with any wind energy experiment are the power generation and efficiency of the devices in the array. The wind turbine models in the arrangements examined here produce approximately the same power in the exit row. Figure 4.23(A) shows the mechanical power for exit row turbines versus angular velocity of the rotor, according to Equation (2.12). The power produced in turbine arrays is more dependent on parameters like spacing, mast height, and streamwise alignment.

According to the research described in [20], the efficiency of a wind farm can be evaluated according to,

$$
\zeta=\frac{P_{\text {real }}}{P_{\text {ideal }}} \times 100 \%,
$$

where $P_{\text {real }}$ is the actual power produced by the devices in an array and $P_{\text {ideal }}$ is what the power would be if each device behaved as though it were in an entrance row. From [10], the park efficiency for the each configuration of wind turbines analyzed in this work can be found in Table 4.1.

These data indicate that although the entrance and exit rows produce approximately the same power (at approximately the same value of $c_{p}$ ), the second and third row turbines behave differently from case to case. The increase in $\zeta$ for counter rotating cases over that of uniform rotation is attributed to an increase of lift on the rotor 


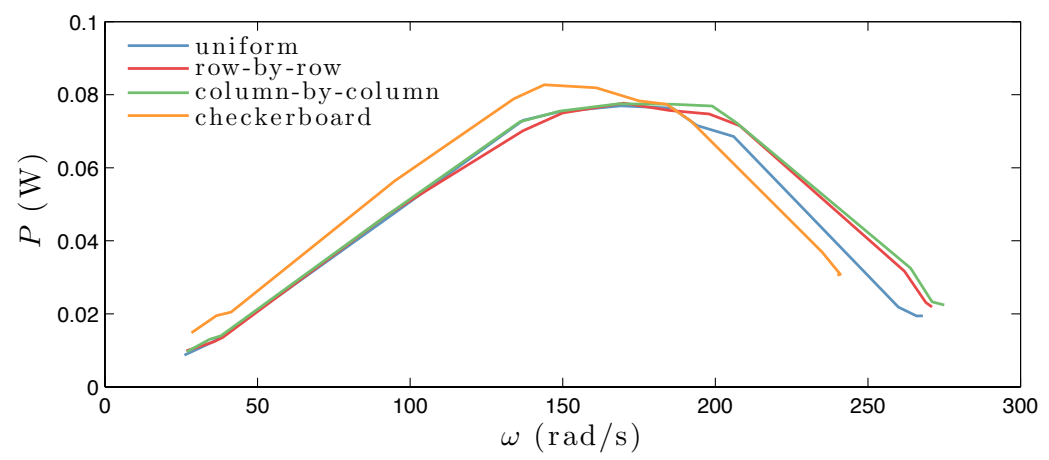

(A) Power curves for exit row turbines.

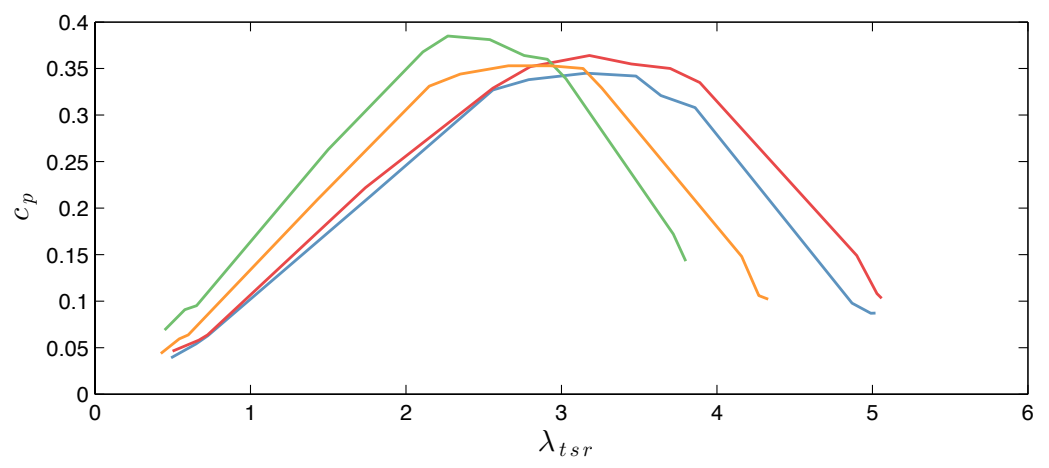

(B) Curves of power coefficient for exit row turbines.

Figure 4.23: Power curves and curves of power coefficient for all exit row turbine models.

Table 4.1: Park efficiency according to array configuration [10].

\begin{tabular}{cc} 
Configuration & $\zeta(\%)$ \\
\hline uniform & 67 \\
row-by-row and & 72 \\
column-by-column & 80 \\
checkerboard & 78 \\
\hline
\end{tabular}


blades due to agreeable rotation in the mean velocity and large scale turbulence acting as inflows for non-leading turbines. Of the non-uniform cases, the checkerboard and column-by-column cases demonstrate the greatest increase in park efficiency.

The increases in these cases indicate that there is wake interaction crossing streamwise columns of a wind farm. The influence of neighboring wakes is significant to park efficiency within the first few rows. As seen in the AIMs of the four test configurations in Figure 4.10, the column-by-column arrangement shows an inflow to the exit row turbine that is significantly more uniform that the other cases. This difference is due to the development of counter-rotating structures on the scale of the spanwise turbine spacing $S_{z}=3 * D$.

Looking to the left column of Figure 4.9, one sees contour plots of $\xi$ corresponding to the inflows of each exit row turbine. Comparing these values to the park efficiencies in Table 4.1 indicates that negative values of $\xi$ augment park efficiency. This suggests that increasing the power of wind turbines and arrays may be aided by conditioning the turbulence in the inflow. A negative correlation between $\zeta$ and $\xi$ would suggest that turbulence that is squeezed in one eigenvalue (oblate characteristic spheroids) tends to increase overall wind park efficiency. 


\section{Chapter 5}

\section{Conclusions}

The present study uses a Cartesian array of wind turbines assembled in the wind tunnel at PSU. Mean velocities show expected trends in the streamwise direction $U$ where momentum deficits in the wakes are clear, and the spanwise direction $W$ where the rotational sense of the rotors is distinguished. The rotation of the turbine rotors is also pronounced in the Reynolds shear stress $-\overline{v w}$.

The normalized Reynolds stress anisotropy tensor $b_{i j}$ is composed from the turbulent stress tensor $\overline{u_{i} u_{j}}$ and the turbulent kinetic energy $k$. The second and third invariants of the tensor $\eta$ and $\xi$ are compared on a case-by-case basis. Although the differences of the invariants observed in the wakes are subtle, the wakes of exit row turbines show some structural dependency of invariants on the rotational sense of the turbine rotors. In the cases of clockwise rotation of the rotor blades, the region of $\xi<0$ in the wakes drifts downward from the hub towards $y / D=0$. The opposite trend is seen for the counter-clockwise rotating blades.

The invariants are averaged in regions of the wakes corresponding to the top rotor tip, hub, and bottom rotor tip of the turbines. Although not physical in that these values do not result from the decomposition of $b_{i j}$, average values of $\eta$ and $\xi$ indicate that the turbulence is most isotropic following the hub and nacelle of the wind turbine. 
The average invariants increase in magnitude moving vertically away from $y / D=1$. The averaged invariants for all cases show that the turbulence following the top rotor tip is highly anisotropic and tends to be stretched in one direction. The far wake of the third row of turbines corresponds to the inflow measurement window for the exit row. In that location, the averaged invariants show a return toward the isotropic state. In the upper part of the turbine canopy, direction of rotation of the turbine rotor is clear. The far wake of CW rotating blades show $\xi<0$ whereas CCW blades exhibit $\xi>0$.

Eigendecomposition of the normalized Reynolds stress anisotropy tensor yields a set of eigenvalues $\lambda_{i}$ and corresponding orthogonal vectors. The eigenvalues represent principle components of $b_{i j}$ at each point within the measurement windows and are used to compose spheroids characteristic of the turbulence in the turbine wakes of the exit row. Analysis reveals significant dependence on wall-normal location in the wake on the characteristic spheroids. The characteristic spheroids of extreme values of invariants vary greatly depending on position in the wake. In agreement with the averaged values of the invariants, characteristic spheroids are drawn following the bottom rotor tip, the hub, and the top rotor tip of the exit row wind turbine in the uniform case. The spheroids show distinct character as oblate and prolate spheroids following bottom and top rotor blades, respectively. Following the hub, the spheroids favor oblate shapes and have smaller radii, corresponding to more isotropy in that area.

The set of vectors associated with the principles components form an orthonormal basis and can be concatenated and treated as a direct cosine matrix. From the direct cosine matrix a set of Euler angles of rotation about the Cartesian coordinate axes are calculated. The orientation of these axis in the wakes depends on the rotational sense of the turbine rotors. Residence of the principle axes in octants of the Cartesian co- 
ordinates are tallied to show that turbulence in the wakes of CW rotating blades favor the second and fifth octants trailing the top rotor tip. The turbulence following CCW rotating blades favor the first and fifth octants near the bottom rotor tip. The principle axes reside primarily in the first, second, fifth, and sixth octants of the Cartesian system (where $y / D>0$ ), indicating that there is less rotation around the $x$ axis than the other two.

A treatment was applied to the Euler angles of rotation making more continuous rotations of the principle axes visible for each exit row turbine wake. Rotations around the $y$ axis demonstrate maximum and minimum values directly above and below hub height in the wake and transitioning toward moderate values vertically outward. About the $x$ and $z$ axes are more clearly differentiated in the treated rotation angles. Rotation in the wakes are more pronounced for CW rotating blades (uniform and checkerboard cases) than for the CCW rotating blades (row-by-row and column-by-column cases).

Recalling that both the eigenvalues and the principle axes derive from the same decomposition, the normalized Reynolds stress anisotropy tensor is mapped in its principle components as a field of spheroids rotated about their respective origins. Comparisons of array configurations demonstrate similar behavior and confirms that turbulence trailing the top tips of the rotors tends to exhibit one large eigenvalue and a prolate characteristic spheroid. Conversely, turbulence trailing the bottom rips of the rotors demonstrates a single small eigenvalue and oblate characteristic spheroids. Noting that perfectly isotropic turbulence requires all eigenvalues to be identically zero, smaller characteristic spheroids are related to more isotropic behavior. In all four measurement cases, the size of the spheroids decrease distinctly as they approach the part of the wake following the nacelle, although they never reach radii equal to zero. The 
exit row turbine of the uniform case shows less isotropy of turbulence in the measurement areas of the column-by-column case, as indicated by the larger spheroids in the fields.

The inflow positions show more uniformity of shape and orientation of spheroids than do the wakes. The return to isotropy indicated by smaller spheroids is more prevalent in array configurations where the rotational sense of the rotors changes in the spanwise direction. The far wake of the third row turbine of the uniform case shows more anisotropy than that of the column-by-column case. The decrease of anisotropy of turbulence indicates an overall organization of turbulence for the column-by-column arrangement of turbines. The complementary rotation of devices in the spanwise sense lend to the increase of park efficiency over that of the uniform configuration rotor blades.

In order to establish connections between the flux of kinetic energy $F_{12}$ and the third invariant of $b_{i j}$, threshold maps are made according to their respective algebraic signs and overlaid. The overlapping regions indicate positive correlations between the two quantities with mismatched signs tending to occur in the hub regions of the wake and near the bottom rotor tips. This relationship implies that when the flux of kinetic energy is vertically downward the turbulence tends toward stretched characteristic spheroids. Similarly, when the flux of kinetic energy is vertically upward turbulence is characterized by compressed spheroids. Regions of mismatching algebraic signs of the two quantities are distinctly above the hub height, where there is a downward flux associated with oblate spheroids, or below the hub, where upward flux is associated with prolate spheroids. The agreement of signs of $F_{12}$ and $\xi$ is more pronounced in the CW cases. The difference in overlap regions is associated with the vertical drift of the 
region of $\xi<0$. For CCW blades this region drifts slightly upward in the wake, opposite of what is seen for the region of $F_{12}<0$.

A similar approach is taken comparing the production of turbulence kinetic energy $P_{12}$ and the second invariant of $b_{i j}$. Both of these quantities are by definition positive, making thresholds somewhat arbitrary. For the production a threshold was selected at $1 \%$ of the maximum value for each wake. The invariant $\eta$ was thresholded at its mean value from each measurement window. Overlaying the threshold maps as above demonstrates that the production of turbulence kinetic energy is highly correlated with anisotropic turbulence. The regions of overlap agree quite well for each exit row turbine wake, regardless of direction of rotation. Both quantities are associated with regions of mean velocity gradients in the wall-normal direction.

Finally the power and efficiency of the exit row turbines are compared. The measured power is consistent in the exit row turbines of all cases. Power is greatest for the checkerboard case, although the power coefficient is highest in the column-bycolumn arrangement. The park efficiency defined by Kang and Meneveau [20] is used to differentiate the test cases. It was shown in measurements by Delucia [10] that the column-by-column and checkerboard cases perform $10 \%$ to $13 \%$ better than the uniform and row-by-row cases. This is an indication that the rotation in the turbulent wakes following wind turbines have a significant effect in the spanwise direction. It is thought that increased park efficiency is due to the formation of large periodic structures forming between columns of the turbine array. The formation of structures on the order of magnitude of the turbine spacing also contributes to the organization of invariants shown in the far wake of the third turbine in the column-by-column case.

The results of the current analysis are applicable to wind farm simulation and 
planning software. The flux of kinetic energy and production of turbulence correlate with the invariants of the normalized Reynolds stress anisotropy tensor indicating that anisotropy cannot be neglected in turbine array design. Increasingly complex arrays are being designed through software with sparing site measurement to mitigate costs. A more complete understanding of possible optimizations of turbine arrays is required to rely on this method of design. The results here establish a basis for comparison or validation for future simulations incorporating rotational sense to array designs. The increase of park efficiency in the column-by-column and checkerboard arrangements indicates that a substantial increase in turbine and array efficiency can be attained through the manufacture and construction of wind turbines rotating in alternate directions. 


\section{Bibliography}

[1] J. F. Ainslie. Calculating the flowfield in the wake of wind turbines. Journal of Wind Engineering and Industrial Aerodynamics, 27:213-224, 1988.

[2] R. Barthelmie, O. Rathmann, S. Frandsen, K. Hansen, E. Politis, J. Prospathopoulos, K. Rados, D. Cabezon, W. Schlez, J. Phillips, A. Neubert, J. G. Schepers, and S. P. van der Pijl. Modelling and measurements of wakes in large wind farms. Journal of Physics: Conference series, 75, 2007.

[3] T. Burton, D. Sharpe, N. Jenkins, and E. Bossanvi. Wind Energy Handbook. John Wiley \& Sons, 2001.

[4] R. B. Cal, J. Lebrón, L. Castillo, H. S. Kang, and C. Meneveau. Experimental study of the horizontally averaged flow structure in a model wind-turbine array boundary layer. Journal of Renewable and Sustainable Energy, 2:013106, 2010.

[5] M. Calaf, C. Meneveau, and J. Meyers. Large eddy simulation study of fully developed wind-turbine array boundary layers. Physics of Fluids, 22:015110, 2010.

[6] L. P. Chamorro and F. Porte-Agel. A wind-tunnel investigation of wind-turbine wakes: Boundary-layer turbulence effects. Boundary Layer Metrology, 132:129149, 2009.

[7] L. P. Chamorro and F. Porté-Agel. Turbulent flow inside and above a wind farm: A wind-tunnel study. Energies, 4(11):1916-1936, 2011.

[8] K.-S. Choi and J. L. Lumley. The return to isotropy of homogeneous turbulence. Journal of Fluid Mechanics, 436(1):59-84, 2001.

[9] A. Crespo, J. Hernandez, and S. Frandsen. Survey of modelling methods for wind turbine wakes and wind farms. Wind Energy, 2:1-24, 1999.

[10] Dominic D. A wind tunnel experiment investigating how varying wind turbine configuration affects power output from model wind farms. Master's thesis, Portland State University, 2013. 
[11] J. Diebel. Representing attitude: Euler angles, unit quaternions, and rotation vectors. Matrix, 2006.

[12] European Wind Energy Association. 2030: the next steps for EU climate and energy policy. Report, September 2013.

[13] S. Frandsen, R. Bathelmie, S. Pryor, Ole Tahmann, S. Larsen, and J. Hojstrup. Analytical modelling of wind speed deficit in large offshore wind farms. Wind Energy, 9:39-53, 2006.

[14] R. Gómez-Elvira, A. Crespo, E. Migoya, F. Manuel, and J. Hernández. Anisotropy of turbulence in wind turbine wakes. Journal of wind engineering and industrial aerodynamics, 93(10):797-814, 2005.

[15] N. Hamilton and R. B. Cal. Wind turbine boundary layer arrays for cartesian and staggered configurations: Part II, low-dimensional representations via the proper orthogonal decomposition. Wind Energy, 2014.

[16] N. Hamilton, R. B. Cal, and M. Melius. Wind turbine boundary layer arrays for cartesian and staggered configurations: Part I, flow field and power measurements. Wind Energy, 2014.

[17] N. Hamilton, H. Suk Kang, C. Meneveau, and R. B. Cal. Statistical analysis of kinetic energy entrainment in a model wind turbine array boundary layer. Journal of Renewable and Sustainable Energy, 4(6):063105-063105, 2012.

[18] A. Jimenez, A. Crespo, E. Migoya, and J. Garcia. Advances in large-eddy simulation of a wind turbine wake. In Journal of Physics: Conference Series, volume 75, page 012041. IOP Publishing, 2007.

[19] Sung Nam Jung, Tae-Soo No, and Ki-Wahn Ryu. Aerodynamic performance prediction of a $30 \mathrm{kw}$ counter-rotating wind turbine system. Renewable Energy, 30(5):631-644, 2005.

[20] H. Suk Kang and C. Meneveau. Direct mechanical torque sensor for model wind turbines. 2010.

[21] S. Leonardi, P. Orlandi, L. Djenidi, and R. A. Antonia. Structure of turbulent channel flow with square bars on one wall. International journal of heat and fluid flow, 25(3):384-392, 2004.

[22] S. Lindenberg, B. Smith, K. O’Dell, et al. 20\% wind energy by 2030. National Renewable Energy Laboratory (NREL), US Department of Energy, Renewable Energy consulting Services, Energetics Incorporated, 2008. 
[23] P. Mestayer. Local isotropy and anisotropy in a high-reynolds-number turbulent boundary layer. Journal of Fluid Mechanics, 125:475-503, 1982.

[24] J. Meyers and C. Meneveau. Optimal turbine spacing in fully developed windfarm boundary layers. Wind Energy, 15:305-317, 2012.

[25] Kyoto Protocol. United nations framework convention on climate change. Kyoto Protocol, Kyoto, 1997.

[26] M. Ragheb and A. M. Ragheb. Wind Turbines Theory-The Betz Equation and Optimal Rotor Tip Speed Ratio. InTech, 2011.

[27] O. Rathmann, S. Frandsen, and R. Barthelmie. Wake modeling for intermediate and large wind farms. 2007.

[28] R. S. Rogallo and P. Moin. Numerical simulation of turbulent flows. Annual Review of Fluid Mechanics, 16(1):99-137, 1984.

[29] J. C. Rotta. Statistische theorie nichthomogener turbulenz. Zeitschrift für Physik, 129(6):547-572, 1951.

[30] W. Z. Shen, V. A. K. Zakkam, J. N. Sørensen, and K. Appa. Analysis of counterrotating wind turbines. In Journal of Physics: Conference Series, volume 75, page 012003. IOP Publishing, 2007.

[31] A. J. Simonsen and P.-Å. Krogstad. Turbulent stress invariant analysis: Clarification of existing terminology. Physics of Fluids, 17:088103, 2005.

[32] R. Smalley, S. Leonardi, R. Antonia, L. Djenidi, and P. Orlandi. Reynolds stress anisotropy of turbulent rough wall layers. Experiments in fluids, 33(1):31-37, 2002.

[33] W. D. Smyth and J. N. Moum. Anisotropy of turbulence in stably stratified mixing layers. Physics of Fluids, 12:1343, 2000.

[34] H. Snel. Review of aerodynamics for wind turbines. Wind Energy, 6(3):203-211, 2003.

[35] S. C. Tedds, R. J. Poole, and I. Owen. Wake characteristics of horizontal axis tidal stream turbines in uniform and non-uniform steady flows. International Conference on Ocean Energy, October 2012.

[36] I. Ushiyama, T. Shimota, and Y. Miura. An experimental study of the two-staged wind turbines. Renewable energy, 9(1):909-912, 1996. 
[37] Frances B Van Cleve and Andrea E Copping. Offshore Wind Energy Permitting: A Survey of US Project Developers. Pacific Northwest National Laboratory, 2010.

[38] L. J. Vermeer, J. N. Sorensen, and A. Crespo. Wind turbine wake aerodynamics. Progress in Aerospace Sciences, 39:457-510, 2003.

[39] J. Whale, K.H. Papadopoulos, C. G. Anderson, C. G Helmis, and D. J. Skyner. A study of the near wake structure of a wind turbine comparing measurements from laboratory and full-scale experiments. Solar Energy, 56(6):621-633, 1996.

[40] World Wind Energy Association. Japanese regions transition to $100 \%$ renewable energy.

[41] World Wind Energy Association. Wind energy international 2011/2012, 2012. 